KMU FORSCHUNG AUSTRIA

Austrian Institute for SME Research

ISI

Fraunhofer Institut System- und Innovationsforschung

\title{
Interimevaluierung von protec 2002+ Das Programm zur Förderung des Technologietransfer
}


Diese Studie wurde im Auftrag des Bundesministeriums für Wirtschaft und Arbeit (BMWA) durchgeführt.

KMU FORSCHUNG AUSTRIA

Austrian Institute for SME Research

(Präsident: em. Univ.-Prof. Dr.Dr.h.c. J. Hanns Pichler, M.Sc.)

Für den Inhalt verantwortlich: Walter Bornett

Verfasserlinnen des Berichts:

Sonja Sheikh (KMFA)

Iris Fischl (KMFA)

Vivien Lo (ISI)

Thomas Stahlecker (ISI)

Petra Wagner-Luptacik (ARC sys)

Simone Archut (ARC sys)

Layout:

Susanne Fröhlich

Die vorliegende Studie wurde nach allen Maßstäben der Sorgfalt erstellt.

Die KMU FORSCHUNG AUSTRIA übernimmt jedoch keine Haftung für Schäden oder Folgeschäden die auf diese Studie oder auf mögliche fehlerhafte Angaben zurückgehen.

Dieses Werk ist urheberrechtlich geschützt. Jede Art von Nachdruck, Vervielfältigung, Verbreitung, Wiedergabe, Übersetzung oder Einspeicherung und Verwendung in Datenverarbeitungssystemen, und sei es auch nur auszugsweise, ist nur mit ausdrücklicher Zustimmung der KMU FORSCHUNG AUSTRIA gestattet. 


\section{Inhaltsverzeichnis}

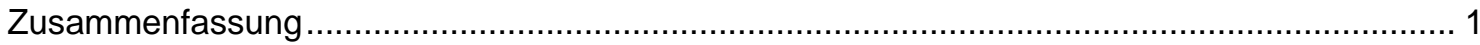

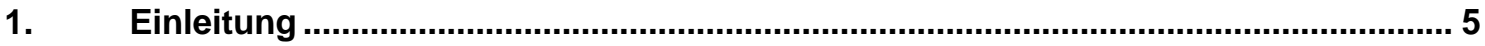

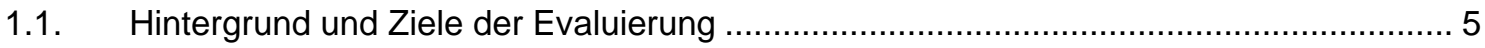

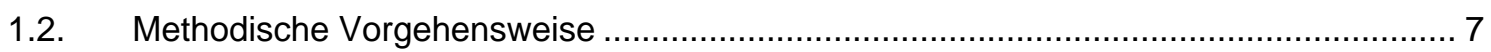

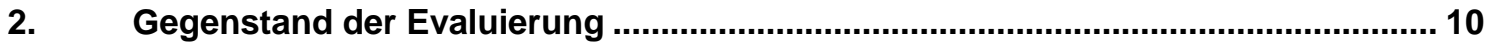

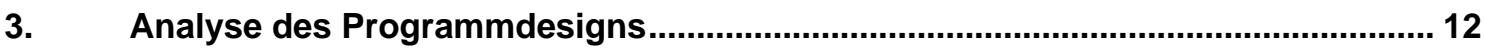

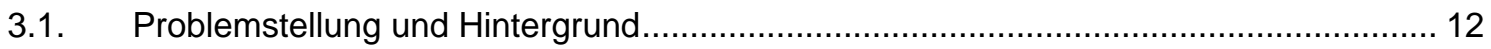

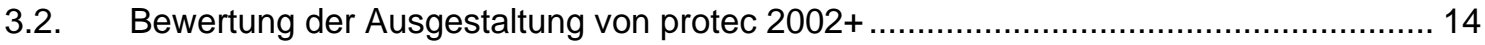

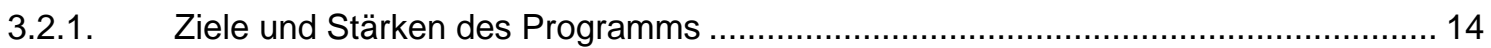

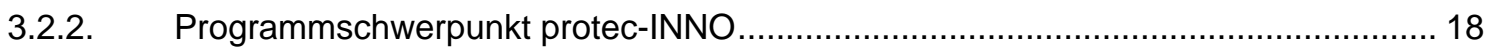

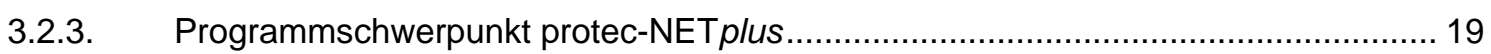

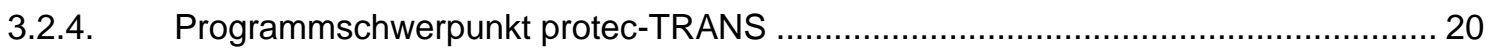

3.3. Einordnung in die österreichische Förderlandschaft ................................................... 21

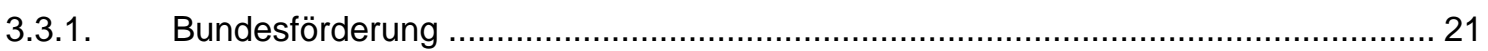

3.3.2. Technologietransferförderung auf der Ebene der Bundesländer ............................... 25

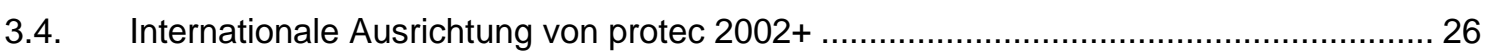

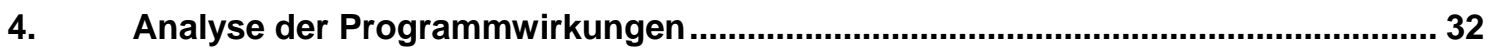

4.1. Zielgruppenerreichung und Struktur der Fördernehmer ......................................... 32

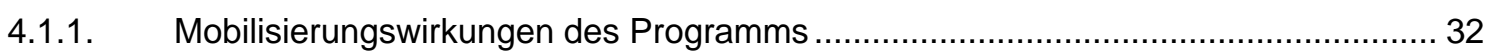

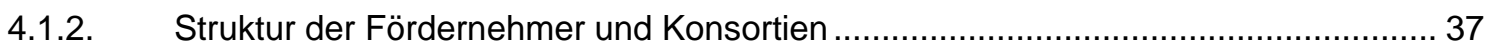

4.1.3. Bestandsaufnahme Gender Mainstreaming ..................................................... 42

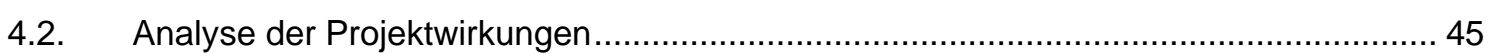

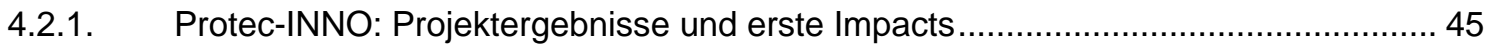

4.2.2. Protec-NETplus: Projektergebnisse und erste Impacts ........................................... 48

4.2.3. Protec TRANS: Projektergebnisse und erste Impacts.............................................. 52

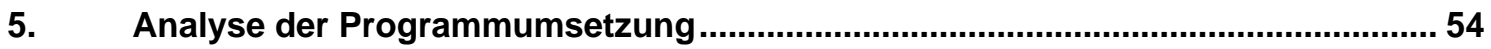

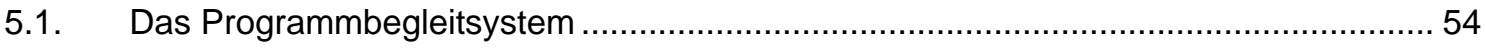

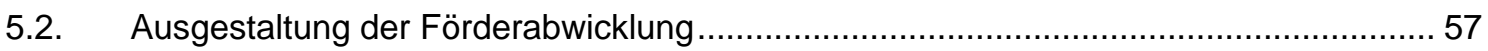

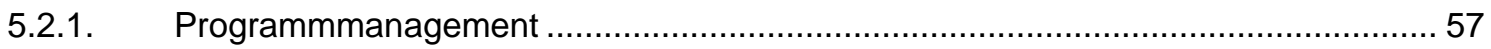

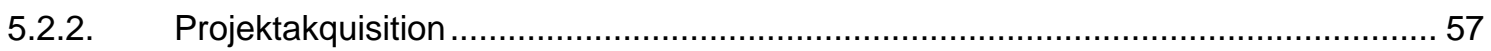

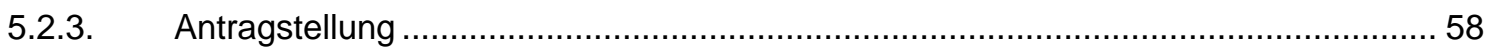

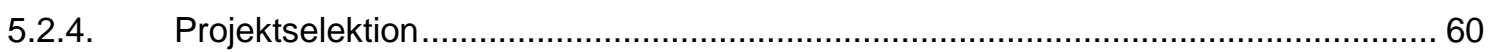

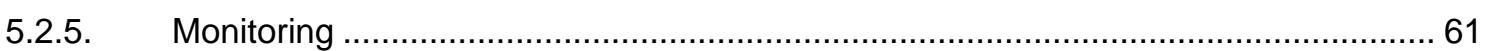

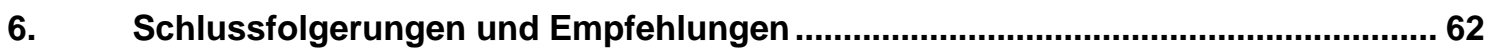

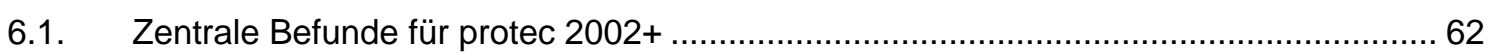

6.2. Herausforderungen für die Weiterentwicklung des Programms .................................. 65

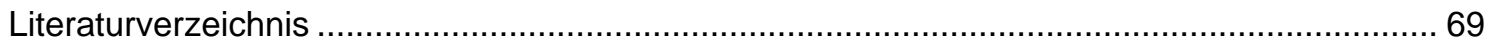

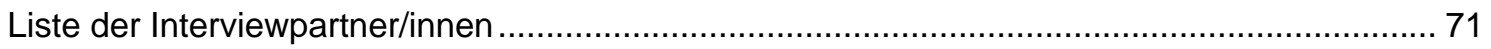




\section{Tabellenverzeichnis}

Tabelle 1: $\quad$ Befragte Programmverantwortliche und ExpertInnen .................................... 8

Tabelle 2: $\quad$ Analysierte Projekte im Rahmen der Befragung der Fördernehmer.................. 9

Tabelle 3: $\quad$ Förderanträge und ausgeschüttete Fördermittel nach

Ausschreibungsrunde ……….......................................................... 11

Tabelle 4: $\quad$ Vergleich ausgewählter österreichischer Netzwerk- und Kooperationsförderprogramme .

Tabelle 5: $\quad$ Übersicht ausgewählter Innovationsnetzwerk- und Innovationskooperationsprogramme anderer Länder

Tabelle 6: $\quad$ Förderanträge und ausgeschüttete Fördermittel nach Ausschreibungsrunde 33

Tabelle 8: $\quad$ Regionale Zuordnung der teilnehmenden Netzwerke

Tabelle 9: $\quad$ Regionale Verteilung der Programmteilnahme nach drei Ausschreibungsrunden, protec-INNO und protec-NETplus

Tabelle 10: Ebenen der Maßnahmengliederung für die Umsetzung von Gender Mainstreaming in der Forschung

Tabelle 11 Frauenbeteiligung an den österreichischen Technologieförderprogrammen

\section{Abbildungsverzeichnis}

Abbildung 1: $\quad$ Vorgehensweise im Rahmen der Zwischenevaluierung von protec $2002+$

Abbildung 2: $\quad$ Bedarf nach Unterstützung von Innovationsaktivitäten

(Mehrfachnennungen möglich), Anteil der Unternehmen in Prozent.

Abbildung 3: Bedarf nach Unterstützung bei Innovationsaktivitäten, Anteil der Unternehmen in Prozent.....

Abbildung $4 \quad$ Verteilung der teilnehmenden Partnertypen in der ersten und zweiten Ausschreibungsrunde, protec-INNO, Anteile in Prozent

Abbildung $5 \quad$ Verteilung der teilnehmenden Partnertypen in der ersten und zweiten Ausschreibungsrunde, protec NETplus, Anteile in Prozent.

Abbildung 6:

Das Programmbegleitsystem von protec $2002+$.... 


\section{Zusammenfassung}

Im vorliegenden Bericht werden die Ergebnisse der Zwischenevaluierung des Programms protec 2002+ - das Programm zur Förderung des Technologietransfers dargestellt, die das Konsortium bestehend aus der KMU FORSCHUNG AUSTRIA (KMFA), dem Fraunhofer Institut System- und Innovationsforschung (ISI) und der ARC systems research GmbH (ARCsys), im Auftrag des Bundesministeriums für Wirtschaft und Arbeit (BMWA) durchgeführt hat. Die Untersuchung erfolgte im Zeitraum September 2005 bis Jänner 2006. Die Zwischenevaluierung basiert im Wesentlichen auf einer qualitativen Analyse der bisherigen Umsetzung und Wirkungen von protec 2002+ auf der Basis von ExpertInneninterviews bzw. Interviews mit Programmverantwortlichen, einer umfangreichen qualitativen Befragung von Fördernehmern und der Analyse verfügbarer Primärdokumente, ergänzt durch eine kleine Bedarfserhebung unter den österreichischen Gewerbe- und Handwerksbetrieben sowie eine Kurzbefragung der Austrian Cooperative Research (ACR) Institute.

Protec 2002+ ist ein Technologietransferprogramm mit dem verstärkt Innovationen in kleinen und mittleren Unternehmen (KMU) ausgelöst bzw. deren Kapazitäten im Bereich Forschung und Entwicklung (FuE) gesteigert werden sollen. Nach der, am 9. April 2002 durch den Rat für Forschung und Technologieentwicklung erfolgten, Empfehlung zur Umsetzung des Programms wurde am 17. Mai 2002 der Startschuss für die Umsetzung gegeben. Für die Gesamtlaufzeit des Programms (2002 - 2006) wurde ein Budget in der Höhe von € 34,8 Mio veranschlagt. Protec 2002+ ist für alle Branchen und Technologiesegmente offen und adressiert neben KMU auch Einrichtungen, die als Know-how Lieferanten für kleine und mittlere Unternehmen aktiv sind. Das umschließt sowohl Forschungseinrichtungen als auch sog. Multiplikatoren, wie spezialisierte Technologietransfereinrichtungen und Unternehmensberater.

Die Umsetzung des Programms erfolgt im Rahmen von drei Programmlinien: „protec-INNO“, „protec-NETplus“ und „protec-TRANS“. Die inhaltiche Klammer von protec 2002+ ist der Technologietransfer und umfasst:

- Projekte, die auf die Verbesserung des unternehmensinternen Innovationsmanagements abzielen (protec-INNO),

- Projekte, die innovative Modelle des Technologietransfers (Kooperationsmodelle, Netzwerke und Plattformen) ausloten und durch ihre überregionale Signalwirkung positive Impulse für die gesamte Technologietransferlandschaft auslösen (protec-NETplus) und

- konkrete Technologietransferprojekte, die auf die effiziente Nutzung externer Ressourcen (Universitäten, außeruniversitäre Forschungseinrichtungen, Fachhochschulen) für $\mathrm{KMU}$ abzielen (protec-TRANS).

Das Programmmanagement der beiden Programmlinien protec-INNO und protec-TRANS erfolgt durch den ERP-Fonds. Für die Abwicklung der Programmlinie protec-NETplus ist die FFG/ Bereich Strukturprogramme verantwortlich. Darüber hinaus wird durch eine sog. MonitoringGruppe, bestehend aus externen ExpertInnen und koordiniert bzw. moderiert von Technopolis Austria, kontinuierlich Feedback zur laufenden Programmumsetzung gegeben und die Feinjustierung des Programms unterstützt.

Bisher wurden drei Ausschreibungsrunden für die Programmlinien protec-INNO und protec NETplus in Form von offenen Calls durchgeführt. Im Rahmen dieser drei Ausschreibungsrunden wurden insgesamt 136 Projekte eingereicht (63 bei protec-INNO und 73 bei protecNETplus) und 49 Projekte gefördert (23 bei protec-INNO und 26 bei protec-NETplus). Bei protec-INNO wurden dabei bisher über alle drei Ausschreibungsrunden hinweg insgesamt $€ 3,25$ Mio an Fördermitteln ausgeschüttet, bei protec-NETplus waren es $€ 4,51$ Mio. Die Programmlinie protec-TRANS wurde am 7. April 2003 nach der Genehmigung durch die Europäische Kommission auf Basis des Gemeinschaftsrahmens für staatliche FuE-Beihilfen gestartet und umfasst bisher 21 erfolgreiche Projekte, die im Rahmen eines Antragsverfahren (open call) gestellt und mit insgesamt € 2,17 Mio gefördert wurden. 
Die Ergebnisse der Zwischenevaluierung des Programms protec 2002+ können folgendermaßen zusammengefasst werden:

Die generelle Ausgestaltung von protec 2002+ bezüglich Ziel- und Schwerpunktsetzung sowie Zielgruppe wird von den befragten Personen (ExpertInnen und Fördernehmern) fast ausnahmslos positiv bewertet. Die zentralen Stärken des Programms lassen sich wie folgt zusammenfassen:

- Protec 2002+ kann bereits in der Beantragungsphase zu wesentlichen Erkenntnissen und Lerneffekten bei den beteiligten KMU führen, da sie sich mit ihren Innovationsbedürfnissen und -kompetenzen auseinandersetzen müssen.

- Die Programmausgestaltung gibt, auch für Kleinstunternehmen, einen Anstoß zur Arbeitsteilung im Innovationsprozess und bietet innen zugleich die Möglichkeit an einem größeren und eventuell interdisziplinären Projekt teilzunehmen. Durch die Einbindung in ein gemeinschaftliches Forschungsprojekt mit entsprechender eigener Investition in FuE-Vorhaben wird zum einen praktische Erfahrung in der Durchführung eigener FuE aufgebaut und weitergegeben sowie durch die Zusammenarbeit ein gewisser Druck auf die Ergebniserzielung ausgeübt.

- Eine wichtige Stärke des Programms ist die Offenheit für unterschiedliche Akteursgruppen und die Unterstützung des Technologietransfers in heterogenen Akteurskonstellationen.

- Die Einbindung von Intermediären gilt als weitere wichtige Stärke von protec 2002+. In ihrer Funktion als Mobilisierer und Multiplikatoren können sie entscheidend dazu beitragen, die Anstoßwirkungen der Fördermaßnahme zu erhöhen, und sind wesentliche Faktoren für die Zielgruppenerreichung.

- Die Ausgestaltung von protec 2002+ als Projektförderung trifft - im Zusammenspiel mit anderen Möglichkeiten des Technologietransfers - die Bedürfnisse der Zielgruppe. Aufgrund der Vielfältigkeit der Ausprägungen, die Technologietransfer haben kann, ist eine ganze Bandbreite von Instrumenten sinnvoll. Die Projektförderung von protec 2002+ füllt hierbei eine wichtige Bedürfnislücke der Zielgruppe.

- Eine wesentliche Stärke des Förderansatzes ist zudem die überregionale Ausrichtung des Programms sowie die Themenoffenheit mit der Möglichkeit der bottom-up Entwicklung neuer und bestehender Technologiefelder.

Da der Bereich Technologietransfer aufgrund seiner Vielfalt ein ausdifferenziertes Förderinstrumentarium notwendig macht, deckt protec 2002+ eine Lücke ab. Für den Technologietransfer im vorwettbewerblichen Bereich füllt protec 2002+ die Lücke zwischen der Forschung/Entwicklung und dem Endprodukt. Über die Einbindung von Technologiezentren und Hochschulen fungiert das Programm auch in der Mobilisierung vorhandener Infrastrukturen. Die FuE- bzw. Technologietransferförderung auf Bundesländerebene zeigt, dass im österreichischen Fördersystem zu protec 2002+ keine nennenswerte Alternative (v. a. im Sinne der Überregionalität, Förderhöhe und Förderdauer) existiert.

Die Analyse der Mobilisierungswirkungen von protec 2002+ hat gezeigt, dass das Technologietransferprogramm vor allem den Unternehmenssektor sehr stark anspricht und dass die beteiligten Klein- und Mittelbetriebe, insbesondere bei protec-NETplus, nicht nur als Pilotanwender bzw. Projektpartner, sondern auch als Konsortialführer eine wichtige Rolle bei protec 2002+ einnehmen. Somit weist das Programm - entsprechend seinen Zielsetzungen - einen eindeutigen KMU-Fokus auf. Darüber hinaus ist die Struktur der Programmteilnehmer sehr heterogen und variiert in den bisherigen drei Ausschreibungsrunden stark, was für die Offenheit des Programms für unterschiedliche Partner- und Netzwerk-Konstellationen spricht. Auch zeigt sich, dass es protec $2002+$ eindeutig gelungen ist, sich erfolgreich als überregionales Technologietransferprogramm zu etablieren. Damit wird das Programm auch der Zielsetzung gerecht, dass vor allem modellhafte Projekte mit überregionaler Signalwirkung initiiert werden sollen. 
Aus Sicht der Fördernehmer stellt die Programmlinie protec-INNO von der Zielsetzung und Konzeption ein gutes Angebot dar, das es in der österreichischen Förderkulisse vorher in dieser Form nicht gegeben hat. Die im Rahmen von protec-INNO geförderten Projekte zielen auf die Entwicklung von Innovationsmanagement-Tools und Beratungsansätzen in Konsortien ab, die darauf folgend durch einzelne Konsortialpartner mit Unternehmen als Pilotanwender getestet werden. Die Konzeption und Entwicklung der geplanten Tools sowie die darauf folgende modellhafte Implementierung konnte bei der überwiegenden Mehrheit der untersuchten Projekte erfolgreich durchgeführt werden. In der Regel wurden dabei jedoch mehr oder minder etablierte Methoden optimiert, adaptiert oder neu kombiniert jedoch wenig völlig neue Ansätze erwirkt. Aus den Beobachtungen lässt sich schließen, dass es sich bei den Ergebnissen der untersuchten protec-INNO Projekte weniger um radikale Innovationen handelt als vielmehr überwiegend um inkrementelle bzw. Anpassungsinnovationen.

Die entwickelten Innovationsmanagement-Tools werden vorwiegend für den „Eigengebrauch“ in einem oder mehreren an den Konsortien beteiligten Beratungsunternehmen bzw. Transferstellen eingesetzt, seltener jedoch darüber hinaus verbreitet. Als wesentliche Auswirkung der Projekte kann bisher demzufolge, neben dem (methodischen) Know-How Aufbau, den die Konsortialpartner für sich erwirken konnten, sowie der Sensibilisierung der beteiligten KMU hinsichtlich des Nutzens von Optionen im Innovationsmanagement, die Erweiterung der Portfolios der Beratungsunternehmen festgestellt werden. Nachvollziehbare Aktivitäten zur Diffusion über das Förderprojekt hinaus lassen sich derzeit nur bei der Minderzahl der analysierten protecINNO Projekte feststellen.

Insgesamt werfen die Analysen zu protec-INNO die Frage auf, ob der Schwerpunkt in der Technologietransferförderung auch zukünftig noch auf der Entwicklung weiterer neuer Innovationsmanagement-Tools liegen sollte. Es gibt Hinweise darauf, dass die Notwendigkeit einer Förderung der Neuentwicklung von Innovationsmanagement-Tools nicht mehr in hohem Maße gegeben ist. Die Programmlinie protec-INNO hat in diesem Bereich in den vergangenen Jahren wertvolle Pionierarbeit geleistet und wichtige Anstöße gegeben. Die Schwerpunktsetzung in der von protec-INNO adressierten Form erscheint jedoch inzwischen ihre Aufgabe erfüllt zu haben. Es sollten jedoch bei einer Weiterentwicklung des Programms protec 2002+ die Stärken von protec-INNO mit übernommen werden. Dazu gehört vor allem die Diffusion und Integration von Innovationsstrategien.

\section{Protec-NETplus}

Die im Rahmen der Evaluierung befragten Fördernehmer gaben ausnahmslos an, mit dem Programmschwerpunkt protec-NETplus in hohem Maße zufrieden zu sein. Dies bezieht sich auf die Zielsetzung und Zielgruppe, die Ausgestaltung, die Förderabwicklung durch die FFG und die Förderintensität. Die zentralen Bedürfnisse der Fördernehmer werden mit dieser Programmlinie deutlich getroffen, die strategische Zielsetzung von protec-NETplus ist nach wie vor gültig. Die Förderung eines Netzwerk- und Kooperationsaufbaus wird auch von ExpertInnen einhellig als eine der wichtigsten Formen des Technologietransfers charakterisiert.

Die technischen Ziele der protec-NETplus Projekte reichen von der Durchführung computergestützter Simulationen, der Erstellung von Planungsgrundlagen für einen spezifischen Technologiebereich und erfolgreich durchgeführten Testverfahren bis hin zur Entwicklung und Optimierung vermarktungsfähiger Produkte. Diese Zielsetzungen wurden in den analysierten abgeschlossenen Projekten größtenteils erreicht, in den sich in der Endphase befindenden Projekten ist die Erreichung in überwiegendem Ausmaß absehbar. Zentrale und als sehr positiv zu beurteilende Lerneffekte, die mit protec-NETplus Projekten erzielt wurden, betreffen dabei vor allem die Netzwerkarbeit. Die Erfahrung des Arbeitens in einem Netzwerk wurde von den befragten Fördernehmern als wichtig eingestuft. Insbesondere bei jenen Projekten in welchen unterschiedliche Institutionen zusammenarbeiteten, zählen der Know-how-Aufbau und -Transfer sowie der Informationsaustausch zu den am stärksten feststellbaren Auswirkungen auf Projektebene. 
Der Bedarf nach einer überregionalen Netzwerkförderung für ein konkretes Innovationsvorhaben, wie protec-NETplus darstellt, scheint entsprechend der Analysen eindeutig gegeben. Vom überwiegenden Teil der ExpertInnen wird der Mehrwert im Bereich protec-Netplus als nach wie vor sehr hoch bewertet, da die Kooperationsbereitschaft zwischen KMU trotz vieler Anstrengungen immer noch als gering einzuschätzen ist. Die Verbindung von Netzwerk- und Innovationsaktivitäten ist zwar anspruchsvoll, angesichts des inzwischen anerkannten Nutzens von Innovationsnetzwerken leitet sich aber gerade daraus ein nach wie vor wichtiger Förderbereich ab. Aus diesen Gründen wird eine Weiterführung der Programmlinie protec-NETplus empfohlen.

\section{Protec-TRANS}

Die befragten Fördernehmer haben den Schwerpunkt protec-TRANS durchwegs positiv hinsichtlich der Erfüllung ihrer Bedürfnisse bewertet. Grundlegendes Element der Programmlinie protec-TRANS ist dabei der Einbezug externer Expertise zur Auslösung substantieller Produktbzw. Verfahrensinnovationen. Die inhaltlichen Zielsetzungen wurden in allen untersuchten Projekten erreicht. Die konkreten Vorhaben (Produkte und Dienstleistungen) wurden plangemäß unter Einbezug externer Expertise entwickelt. Diese Expertise wurde in Form von Beratung, universitären Forschungsarbeiten, spezifischem technologischem Know-how und technischer Infrastruktur in die Projekte eingebracht und hat in den meisten Fällen einen wesentlichen Beitrag zur Erreichung der Projektergebnisse geliefert.

Darüber hinaus zeichnet sich eine sehr positiv zu beurteilende nachhaltige Wirkung der Förderung im Bereich des Kooperationslernens bei den analysierten Projekten ab. Als großer Erfolg der Programmlinie protec-TRANS ist auch zu werten ist, dass die Ergebnisse aus zwei untersuchten Projekten entsprechend patentiert werden konnten.

Insgesamt kann die Förderlinie protec-TRANS als unkomplizierte Einstiegsmöglichkeit für KMU in den Technologietransfer charakterisiert werden. Für eine ganze Reihe der befragten Unternehmen wurde durch die Teilnahme an protec-TRANS zum ersten Mal die Zusammenarbeit mit einer Universität oder Forschungseinrichtung realisiert. Aus diesen Gründen wird eine Weiterführung der Programmlinie protec-TRANS empfohlen.

Folgende Herausforderungen ergeben sich für die Weiterführung des Programms:

Bei protec 2002+ handelt es sich um ein erfolgreiches Förderprogramm, das mit dem Thema Technologietransfer eine zentrale Funktion in der österreichischen Förderlandschaft erfüllt. Dementsprechend wird eine Weiterführung des Förderprogramms über den vorgesehenen Endzeitpunkt in 2006 empfohlen. Die mit einer möglichen Weiterführung verbundenen Herausforderungen sind im Folgenden kurz zusammengefasst:

- Weiterentwicklung der thematischen Schwerpunkte von protec 2002+

- Nutzung von Synergiepotenzialen zu anderen Programmen mit Netzwerklinien

- Förderung der Diffusion und des Markteintritts

- Unterstützung der Internationalisierung der Fördernehmer

- Weiterentwicklung der Promotion des Programms

- Vereinheitlichung des Monitoringsystems

Abschließend ist festzuhalten, dass protec 2002+ eine zentrale Funktion darin einnimmt, KMU den Technologietransfer als Option für die Ausweitung ihrer Innovationsaktivitäten näher zu bringen. Das Förderinstrument kann hierbei deutliche Erfolge in der Verringerung von Hemmnissen dieser Zielgruppe vorweisen. Vor dem Hintergrund, dass auch in Zukunft der wissensund innovationsbasierte Wettbewerb weiter deutlich zunehmen wird, und damit die Bedeutung für kleine und mittlere Unternehmen, in einem größeren Ausmaß mit Wissensinstitutionen zu kooperieren, steigen wird, erscheint eine weitere Förderung von Technologietransfer jedenfalls angebracht. 


\section{Einleitung}

\subsection{Hintergrund und Ziele der Evaluierung}

Die Bedeutung von Innovationen für die zukünftige Wettbewerbsfähigkeit entwickelter Volkswirtschaften steht inzwischen außer Frage. Dabei kommt insbesondere den Innovationsaktivitäten in kleinen und mittleren Unternehmen (KMU) eine besondere Rolle zu. Erstens können sie häufig flexibler als Großunternehmen auf Technologie- und Marktveränderungen reagieren und damit "windows of opportunity" wahrnehmen und zweitens kommt innen insbesondere für die österreichische Volkswirtschaft eine besondere Bedeutung zu. ${ }^{1}$ Gleichzeitig verfügen KMU jedoch häufig nicht über die notwendigen Kapazitäten und Kompetenzen, um regelmäßige Innovationsaktivitäten zu betreiben. An dieser Stelle setzt die öffentliche Innovationsförderung an, zu deren Instrumentarium "protec 2002+ - Das Programm zur Förderung des Technologietransfers" gehört. Das Ziel von protec 2002+ ist es dementsprechend, Innovationen in KMU auszulösen, ihre FuE-Aktivitäten zu verstetigen und FuE-Kapazitäten auszubauen.

Die Innovationspolitik steht dabei zunehmend vor einer dreifachen Herausforderung: Sie muss erstens geeignete Zielsetzungen und Instrumente für die Anpassung und Modernisierung des Innovationssystems bereitstellen, zweitens ihre Interventionen im Geflecht der vielfältigen Akteure abstimmen und sie muss dies drittens auf absehbare Zeit unter der Bedingung knapper werdender staatlicher Mittel tun. Hierbei spielt auch die ab 2007 aufgrund der EU-Osterweiterung absehbare Veränderung der Mittelverfügbarkeit der Europäischen Strukturfonds (vor allem EFRE) eine Rolle. ${ }^{2}$ Damit werden eine weitergehende Priorisierung und eine kontinuierliche Bewertung der bisherigen Innovationspolitik unumgänglich.

Vor diesem Hintergrund hat das Bundesministerium für Wirtschaft und Arbeit (BMWA) das Konsortium bestehend aus der KMU FORSCHUNG AUSTRIA (KMFA), dem Fraunhofer Institut System- und Innovationsforschung (ISI) und der ARC systems research GmbH (ARCsys) mit der Zwischenevaluierung von protec 2002+ - Dem Programm zur Förderung des Technologietransfers beauftragt. Ziel der Zwischenevaluierung ist die Analyse des Programms und des bisherigen Programmverlaufs von protec 2002+ mit den drei Programmlinien protec-INNO, protec-NETplus und protec-TRANS. Da die erste Ausschreibung von protec-INNO und protecNETplus bereits 2002 erfolgte, kann zum jetzigen Zeitpunkt ein erstes Zwischenfazit gezogen werden, um die Ausrichtung, Zielerreichung und Zielgruppenereichung des Programms zu bewerten und etwaige Korrekturen im Hinblick auf die Weiterentwicklung des Förderinstrumentariums zu empfehlen. Den Bewertungsrahmen bilden dabei nicht nur die Effektivität und Effizienz des Programms und seiner drei Linien selbst, sondern auch die Einordnung in das weitere österreichische und internationale Technologietransferunterstützungssystem. In der vorliegenden Zwischenevaluierung werden demnach folgende Themenfelder bearbeitet:

\section{- Programmdesign:}

Schwerpunktsetzung und Förderlinien innerhalb des Programms;

- Signifikanz und Passfähigkeit:

Positionierung in der österreichischen Förderlandschaft und im europäischen

Förderkontext;

- Umsetzung:

Programmmanagement und Effizienz der Abwicklung;

- Struktur der Fördernehmer und Zielgruppe:

Mobilisierungswirkung des Programms, Bestandsaufnahme Gender Mainstreaming;

\footnotetext{
99,6 \% aller österreichischen Unternehmen haben weniger als 250 Beschäftigte.

Siehe auch Kaufmann / Wagner-Luptacik (2005)
} 


\section{- Programmwirkungen:}

Projektergebnisse und erste Impacts;

- Weiterentwicklung:

Empfehlungen für Änderungen in Programmdesign und -umsetzung.

In neuerer Zeit werden von Programmverantwortlichen zur Bewertung staatlicher Interventionsmaßnahmen verstärkt begleitende bzw. Interimevaluierungen eingesetzt. Dies hat den Vorteil, dass strategische Intelligenz frühzeitig in die Programmsteuerung einfließen kann. Die Aussagefähigkeit von Interimsevaluierungen wird jedoch dadurch eingeschränkt, da zum Bewertungszeitpunkt die Programmwirkungen häufig noch nicht in vollem Umfang eingetreten sind. Davon ist auch im vorliegenden Fall der Untersuchung des Technologietransferprogramms protec 2002+ auszugehen. In Bezug auf die Analyse die obigen Themenfelder sei deshalb darauf hingewiesen, dass im Zuge der gegenständlichen Zwischenevaluierung nur die entsprechend kurzfristigen Umsetzungsergebnisse und Auswirkungen erfasst und analysiert werden können. Bisher wurde lediglich ein Viertel der insgesamt 75 in den drei Programmlinien von protec 2002+ geförderten Projekte abgeschlossen. Dabei handelt es sich ausschließlich um Projekte der ersten Ausschreibungsrunde von protec-INNO und protec-NETplus bzw. um einige der frühen protec-TRANS Projekte. Die Projekte der zweiten und dritten Ausschreibungsrunde laufen größtenteils noch bzw. stehen erst kurz vor dem Abschluss. Eine Umsetzung der jeweiligen Projektergebnisse in marktfähige Produkte und Dienstleistungen bzw. eine breite Diffusionswirkung der entwickelten Methoden kann daher noch nicht erwartet werden. Mögliche Anzeichen in Hinblick auf die mittel- bzw. längerfristigen Auswirkungen der geförderten Projekte können jedoch zum gegebenen Zeitpunkt bereits einen wichtigen Input für mögliche Weichenstellungen und Korrekturen in der zukünftigen Programmgestaltung sowie in Bezug auf das zukünftige Programmdesign geben.

Im folgenden Kapitel (1.2) wird demzufolge die methodische Vorgehensweise, die der vorliegenden Zwischenevaluierung zugrunde liegt, beschrieben und Kapitel 2 gibt das zu evaluierende Programm in seinen Grundzügen wieder und liefert einen kurzen Überblick über den derzeitigen Status Quo von protec 2002+. Erste Ergebnisse in Bezug auf die Analyse des Programmdesigns werden in Kapitel 3 dargestellt. Kapitel 4 gibt die Analyse der bisherigen Programmwirkungen wieder und Kapitel 5 widmet sich der Analyse der Programmumsetzung. Kapitel 6 fasst schließlich die wichtigsten Ergebnisse der Zwischenevaluierung zusammen und leitet entsprechende Schlussfolgerungen und Empfehlungen in Hinblick auf die zukünftige Programmgestaltung und -umsetzung ab. 


\subsection{Methodische Vorgehensweise}

Die Bearbeitung der oben genannten Themenfelder im Rahmen der Zwischenevaluierung basiert im Wesentlichen auf einer qualitativen Analyse der bisherigen Umsetzung und Wirkungen von protec 2002+ auf der Basis von ExpertInneninterviews bzw. Interviews mit Programmverantwortlichen, einer umfangreichen Befragung von Fördernehmern und der Analyse verfügbarer Primärdokumente, ergänzt durch eine kleine Bedarfserhebung unter den österreichischen Gewerbe- und Handwerksbetrieben sowie eine Kurzbefragung der Austrian Cooperative Research (ACR) Institute. Abbildung 1 gibt einen Überblick über die Vorgehensweise im Rahmen der Evaluierung.

Abbildung 1: Vorgehensweise im Rahmen der Zwischenevaluierung von protec 2002+

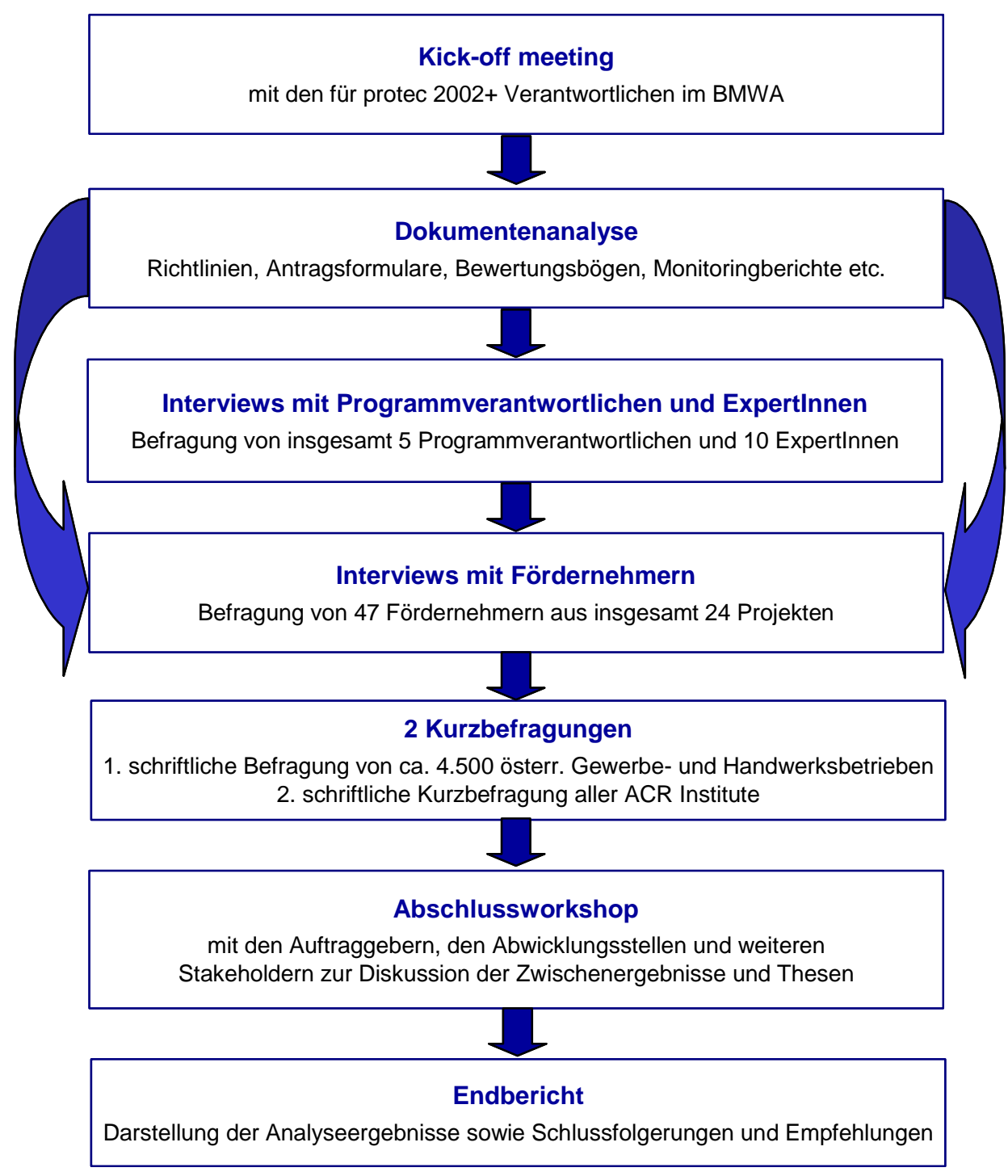

Den Auftakt für die Evaluierung bildete ein Kick-off Meeting mit den VertreterInnen des auftraggebenden Ministeriums, zu dem auch die Programmverantwortlichen Stellen geladen waren. Ziel des Kick-off Meetings war einerseits der Austausch von Informationen und Erwartungen hinsichtlich der Evaluierung sowie die methodische und inhaltliche Konkretisierung der Evaluierungsaktivitäten, andererseits wurden im Rahmen des Kick-off Meetings bereits erste relevante Informationen in Bezug auf die Hintergründe, Ziele und Funktionsweisen von protec $2002+$ erhoben. 
Die Dokumentenanalyse erfolgte über die gesamte Laufzeit der Evaluierung parallel zu den anderen Evaluierungsschritten. Bestandteil der Dokumentenanalyse war dabei neben einer Auswertung der von den Abwicklungsstellen zur Verfügung gestellten Programmdokumente (Konzepte, Richtlinien, Antragsformulare, Bewertungsbögen, Zwischen- und Endberichte der geförderten Projekte etc.) sowie der jährlichen Monitoringberichte auch eine erweiterte desk research zur Einordnung von protec 2002+ in den nationalen und internationalen Förderrahmen im Bereich Technologietransferförderung.

Da protec 2002+ durch die Einbindung unterschiedlicher und z. T. sehr heterogener Akteursgruppen gekennzeichnet ist (siehe Kapitel 4.1.2), bildeten darüber hinaus die Interviews mit den Programmverantwortlichen und ExpertInnen des österreichischen Innovationssystems einen wesentlichen Bestandteil der Evaluierung, insbesondere in Hinblick auf die Analyse des Programmdesigns, des Programmmanagements und der Positionierung von protec 2002+ in der österreichischen Förderlandschaft und im europäischen Förderkontext. Tabelle 1 gibt einen Überblick über die in diesem Zusammenhang befragten Akteursgruppen, wobei insgesamt 15 Personen im Rahmen von z. T. mehreren Interviews befragt wurden (für eine Liste der Interviewpartner siehe Anhang).

Tabelle 1: $\quad$ Befragte Programmverantwortliche und ExpertInnen

\begin{tabular}{l|l|c}
\hline & \multicolumn{1}{|c|}{ Bereich } & \multicolumn{1}{c}{ Anzahl } \\
\hline Programmverantwortliche & verantwortliches Bundesministerium & 2 \\
& Programmabwicklungsstellen & 2 \\
„programmunabhängige“ ExpertInnen & begleitendes Monitoring & 1 \\
& $\begin{array}{l}\text { Nationale Förderstellen / Programmverantwort- } \\
\text { liche im Technologie- und Innovationsbereich }\end{array}$ & 4 \\
& Regionale Förderstellen im Technologie- und & 3 \\
& Innovationsbereich & 2 \\
& KMU-Experten & 1 \\
\hline \hline Gesamt & Gender Mainstreaming & 15 \\
\hline
\end{tabular}

In Hinblick auf die Analyse der bisherigen Programmwirkungen (d.h. Projektergebnisse und erste Impacts) sowie die Zufriedenheit mit dem Programm bzw. dem Programmmanagement aus Sicht der Fördernehmer wurden Interviews mit insgesamt 47 Fördernehmern durchgeführt. Diese 47 Fördernehmer waren an insgesamt 24 Projekten beteiligt, d. h. im Durchschnitt wurden 2 Organisationen pro gefördertem Projekt befragt, i.d.R. jeweils der Projektkoordinator sowie ein weiterer Projektpartner. Die Auswahl der zu analysierenden Projekte erfolgte nach Ausschreibungsrunde und Programmlinie, dabei wurden jene Projekte bevorzugt behandelt, deren Beginn bereits länger zurücklag. Aufgrund der geringen Zeitspanne seit Beginn des Programms im Jahr 2002 sind wirtschaftliche Effekte im Sinne einer Umsetzung der Projektergebnisse in marktfähige Produkte und Dienstleistungen erst in geringem Maße zu erwarten und dann vor allem bei Projekten der ersten Ausschreibungsrunde. Um jedoch einen zu starken Bias zugunsten der ersten Ausschreibungsrunde von protec 2002+ zu vermeiden, wurden auch Projekte der zweiten Ausschreibungsrunde in die Analyse mit einbezogen. Folgende Tabelle 2 zeigt die Auswahl der Projekte, die im Rahmen der vorliegenden Zwischenevaluierung näher analysiert wurden. 
Tabelle 2: $\quad$ Analysierte Projekte im Rahmen der Befragung der Fördernehmer ${ }^{*}$

\begin{tabular}{l|c|c|c}
\hline & $\begin{array}{c}\text { erste } \\
\text { Ausschreibungsrunde }\end{array}$ & $\begin{array}{c}\text { zweite } \\
\text { Ausschreibungsrunde }\end{array}$ & Gesamt \\
\hline protec-INNO & $\mathbf{8}$ (von insgesamt 9) & $\mathbf{4}$ (von insgesamt 8) & $\mathbf{1 2}$ (von 17) \\
protec-NETplus & $\mathbf{3}$ (von insgesamt 5) & $\mathbf{4}$ (von insgesamt 6) & $\mathbf{7}$ (von 11) \\
Protec-TRANS & $\mathbf{5}$ (von insgesamt 21) & $\mathbf{5}$ (von 21) \\
\hline \hline Gesamt & & & $\mathbf{2 4}$ (von 49) \\
\hline${ }^{\star}$ Stand: Juni 2005 & \multicolumn{3}{l}{} \\
\hline
\end{tabular}

Ergänzend zu den obigen Analysemethoden wurden im Rahmen der Zwischenevaluierung zwei Kurzbefragungen durchgeführt. In der ersten Kurzbefragung ging es darum, die Bekanntheit von protec 2002+ sowie die Innovationsbedürfnisse der österreichischen Gewerbe- und Handwerksbetriebe zu erheben, die eine der Zielgruppen des Programms darstellen. Zu diesem Zweck wurden im Rahmen der vierteljährlichen Konjunkturbeobachtung der KMU FORSCHUNG AUSTRIA unter knapp 4.000 Gewerbe- und Handwerksbetrieben zwei entsprechende Zusatzfragen gestellt. Zwar wird dadurch nicht die gesamte Zielgruppe von protec $2000+$ abgebildet, da jedoch knapp 30 \% aller österreichischen KMU der Sparte Gewerbe- und Handwerk zuzuordnen sind, ${ }^{3}$ gibt die Befragung einen guten Hinweis auf den Unterstützungsbedarf bei KMU im Bereich der öffentlichen Technologietransferförderung. Darüber hinaus wurde eine schriftliche Kurzbefragung unter den kooperativen Forschungseinrichtungen (ACR) durchgeführt, die insbesondere Aufschluss über die Gründe der Beteiligung bzw. Nicht-Beteiligung der ACR Institute an protec 2002+ als wichtige Intermediäre für KMU im Bereich der angewandten außeruniversitären Forschung geben sollte.

Die Ergebnisse der durchgeführten Evaluierungsschritte wurden im Rahmen von zwei internen Workshops zusammengeführt, analysiert und in Form von Thesen und Szenarien insbesondere in Hinblick auf die Weiterführung von protec 2002+ aufbereitet und im Rahmen eines Abschlussworkshops mit den Programmverantwortlichen und weiteren Stakeholdern von protec 2002+ diskutiert. Kommentare, Unterstützungen, Abgleiche und kritische Anmerkungen der Workshop-TeilnehmerInnen wurden in den vorliegenden Endbericht aufgenommen beziehungsweise zur Argumentationsschärfung berücksichtigt.

3 Von den verbleibenden $70 \%$ sind etwa $27 \%$ im Handel, $19 \%$ in der Tourismus und Freizeitwirtschaft, $16 \%$ im Bereich Information und Consulting, $6 \%$ im Transport und Verkehr und $2 \%$ in der Industrie tätig. 


\section{Gegenstand der Evaluierung}

Insbesondere kleine und mittlere Unternehmen (KMU) sind bei der Anwendung neuer Techniken sowie der Entwicklung und Vermarktung neuer Sach- und Dienstleistungen mit Ressourcenengpässen konfrontiert. Um diese Defizite auszugleichen, gewinnt die Erschließung externer Quellen für KMU zunehmend an Bedeutung. Mit dem Technologietransferprogramm protec 2002+ sollen verstärkt Innovationen in kleinen und mittleren Unternehmen ausgelöst bzw. deren Kapazitäten im Bereich Forschung und Entwicklung (FuE) gesteigert werden. Der Rat für Forschung und Technologieentwicklung hat das Programm am 9. April 2002 zur Umsetzung empfohlen. Mit der darauf folgenden Genehmigung der Richtlinien durch den zuständigen Bundesminister am 17. Mai 2002 wurde der Startschuss für die Umsetzung gegeben. Für die Gesamtlaufzeit des Programms $(2002$ - 2006) wurde ein Budget in der Höhe von $€ 34,8$ Mio veranschlagt.

Protec 2002+ ist für alle Branchen und Technologiesegmente offen und adressiert neben mittelständischen Unternehmen auch Einrichtungen, die als Know-how Lieferanten für kleine und mittlere Unternehmen aktiv sind. Das umschließt sowohl Forschungseinrichtungen als auch sog. Multiplikatoren, wie spezialisierte Technologietransfereinrichtungen und Unternehmensberater. Die inhaltliche Klammer von protec 2002+ ist der Technologietransfer. Dieser umfasst:

- Projekte, die auf die Verbesserung des unternehmensinternen Innovationsmanagements abzielen sowie

- Projekte, die innovative Modelle des Technologietransfers (Kooperationsmodelle, Netzwerke und Plattformen) ausloten und durch ihre überregionale Signalwirkung positive Impulse für die gesamte Technologietransferlandschaft auslösen,

- konkrete Technologietransferprojekte, die auf die effiziente Nutzung externer Ressourcen (Universitäten, außeruniversitäre Forschungseinrichtungen, Fachhochschulen) für KMU abzielen.

Die Umsetzung von protec 2002+ erfolgt im Rahmen von drei Programmlinien:

protec-INNO

Das übergeordnete Ziel der Programmlinie protec-INNO ist die Verbesserung des unternehmensinternen Innovationsmanagements. Projekte in dieser Programmlinie haben die Entwicklung, Diffusion und Implementierung von geeigneten Innovationsmanagement-Instrumenten, sowie die Entwicklung, Erprobung und Anwendung neuer Beratungsansätze und -mechanismen (Good Practice Modelle) und die Verbreitung von Tools und Good Practice Modellen zur Unterstützung der Beschleunigung und Professionalisierung der ökonomischen Verwertung geistigen Eigentums zum Gegenstand.

protec-NETplus

Mit protec-NETplus werden Projekte gefördert, die eine Anhebung des Innovationsniveaus (neue Produkte, Verfahren und Produktionslinien sowie Marktneuheiten) von Unternehmen (v.a. KMU) im Wege von Kooperationsmodellen, Netzwerken und Plattformen zum Ziel haben. Im Vordergrund stehen bei protec-NETplus Projekte, die innovative und modellhafte Formen des Technologietransfers aufzeigen. Die geförderten Projekte sollen Demonstrationscharakter mit überregionaler Signalwirkung haben.

protec-TRANS

Ziel der Programmlinie protec-TRANS ist die Verbesserung der Nutzung externer Ressourcen für KMU, insbesondere die Verbesserung des Zugangs zur Expertise in Forschungseinrichtungen (Universitäten, außeruniversitären Forschungseinrichtungen, Fachhochschulen) zur Auslösung von substantiellen Produkt- und Verfahrensinnovationen und Dienstleistungen sowie zum Aufbau von Forschungs- und Entwicklungskapazitäten. 
Das Programmmanagement der beiden Programmlinien protec-INNO und protec-TRANS erfolgt durch den ERP-Fonds. Für die Abwicklung der Programmlinie protec-NETplus ist die FFG/Bereich Strukturprogramme verantwortlich. Um den programmverantwortlichen Stellen kontinuierlich Feedback zur laufenden Programmumsetzung zu geben und bei der Feinjustierung des Programms zu unterstützen wurde darüber hinaus eine sog. Monitoring-Gruppe eingerichtet. Die in die Monitoringgruppe eingebundenen externen Expertlnnen sollen insbesondere die Außenwahrnehmung des Programms einbringen und die unabhängige Bewertung des Umsetzungsfortschritts gewährleisten. Die Koordination und Moderation der Monitoringgruppe obliegt Technopolis Austria (siehe auch Kapitel 5.

Bisher wurden drei Ausschreibungsrunden für die Programmlinien protec-INNO und protec NETplus in Form von offenen Calls durchgeführt. Im Rahmen dieser drei Ausschreibungsrunden wurden insgesamt 136 Projekte eingereicht (63 bei protec-INNO und 73 bei protecNETplus) und 49 Projekte gefördert (23 bei protec-INNO und 26 bei protec-NETplus). Bei protec-INNO wurden dabei bisher über alle drei Ausschreibungsrunden hinweg insgesamt $€$ 3,25 Mio. an Fördermitteln ausgeschüttet, bei protec-NETplus waren es $€ 4,51$ Mio. Tabelle 3 gibt die Entwicklung der Förderanträge und Fördermittel bei protec-INNO und protec-NETplus nach Ausschreibungsrunde wieder.

Tabelle 3: $\quad$ Förderanträge und ausgeschüttete Fördermittel nach Ausschreibungsrunde

\begin{tabular}{|c|c|c|c|c|}
\hline & \multicolumn{2}{|c|}{ protec-INNO } & \multicolumn{2}{|c|}{ protec-NETplus } \\
\hline & eingereicht & gefördert & eingereicht & gefördert \\
\hline 1. Ausschreibungsrunde & \multicolumn{2}{|c|}{ 15. Sept. bis 16. Dez. 2002} & \multicolumn{2}{|c|}{ 1. Sept. bis 29. Nov. 2002} \\
\hline $\begin{array}{l}\text { Anzahl der Förderanträge } \\
\text { Fördermittel in } €\end{array}$ & 28 & $\begin{array}{c}9 \\
1.161 .944\end{array}$ & 27 & $\begin{array}{c}5 \\
969.157\end{array}$ \\
\hline 2. Ausschreibungsrunde & \multicolumn{4}{|c|}{ 3. Nov. 2003 bis 30. Jan. 2004} \\
\hline $\begin{array}{l}\text { Anzahl der Förderanträge } \\
\text { Fördermittel in } €\end{array}$ & 20 & $\begin{array}{c}8 \\
1.275 .168 \\
\end{array}$ & 17 & $\begin{array}{c}6 \\
1.093 .396 \\
\end{array}$ \\
\hline 3. Ausschreibungsrunde & \multicolumn{4}{|c|}{ 2. Nov. 2004 bis 31. Jan. 2005} \\
\hline $\begin{array}{l}\text { Anzahl der Förderanträge } \\
\text { Fördermittel in } €\end{array}$ & 15 & $\begin{array}{c}6 \\
815.752\end{array}$ & 29 & $\begin{array}{c}15 \\
2.450 .065\end{array}$ \\
\hline $\begin{array}{l}\text { Summe Förderanträge } \\
\text { Summe Fördermittel }\end{array}$ & 63 & $\begin{array}{c}23 \\
3.252 .864\end{array}$ & 73 & $\begin{array}{c}26 \\
4.512 .618\end{array}$ \\
\hline
\end{tabular}

Die Programmlinie protec-TRANS wurde am 7. April 2003 nach der Genehmigung durch die Europäische Kommission auf Basis des Gemeinschaftsrahmens für staatliche FuE-Beihilfen gestartet und umfasst bisher 21 erfolgreiche Projekte, die im Rahmen eines Antragsverfahren (Open Call) gestellt und mit insgesamt $€ 2,17$ Mio gefördert wurden. ${ }^{4}$ 


\section{Analyse des Programmdesigns}

\subsection{Problemstellung und Hintergrund}

Technologietransfer ist ein wesentliches Instrument zur Erhöhung der Wirksamkeit von Innovationssystemen. Er trägt zur wirtschaftlichen Verwendung von FuE-Ergebnissen bei, stärkt die Innovationskraft von Unternehmen und unterstützt FuE-Einrichtungen beim Erkennen industrieller FuE-Problemstellungen sowie beim Rückfluss von Erfahrungen aus der Nutzung der FuE-Ergebnisse. Besonders für kleine und mittlere Unternehmen ist Technologietransfer aufgrund der geringen eigenen FuE-Potenziale und der begrenzten Finanzierungskraft unumgänglich. Die Aneignung von externem technologischen Wissen ist notwendig, um Innovationen hervorbringen und vermarkten zu können. ${ }^{5}$

Das österreichische Innovationssystem ist durch eine Vielzahl kleiner und kleinster Unternehmen gekennzeichnet, die angesichts des zunehmenden internationalen Konkurrenzdrucks externe Technologiequellen benötigen, um langfristig auf den Märkten mit neuen Produkten und Dienstleistungen zu bestehen. Obgleich der österreichische Unternehmenssektor seine Ausgaben im Bereich FuE in den letzten Jahren deutlich gesteigert hat, besteht nach wie vor ein Nachholbedarf im Vergleich zu Ländern wie Finnland, Deutschland, Japan oder den USA, wenn Österreich einen signifikanten Beitrag zur Erreichung des „wettbewerbsfähigsten und dynamischsten wissensbasierten Wirtschaftsraum der Welt" in Europa leisten soll ${ }^{6}$

Vor diesem Hintergrund ist das übergeordnete Ziel von protec 2002+ - dem Programm zur Förderung des Technologietransfers, das in der "Stärkung der Innovationskraft und Hebung der FuE-Leistung der Unternehmen der mittelständischen Wirtschaft (v. a. KMU)"7 besteht, nach wie vor von hoher Bedeutung für das österreichische Innovationssystem. Allgemein wird unter Technologietransfer eine Vielzahl von Aktivitäten und Interaktionen zwischen verschiedenen Akteuren subsumiert. Diese lassen sich grob in zwei Arten von Aktivitäten unterscheiden:

- Technologietransfer zwischen Wissenschaft und Wirtschaft: Übertragung und Anwendung wissenschaftlichen Wissens auf kommerzielle Zwecke.

- Technologietransfer innerhalb der Wirtschaft: Übertragung von Technologie von einem Anwendungs-/Verwendungskontext in einen anderen.

Der Technologietransfer innerhalb der Wirtschaft kann sowohl vertikal, d.h. zwischen Kunde und Zulieferer, als auch horizontal, also zwischen Unternehmen ähnlicher Wertschöpfungsstufen, oder zwischen Unternehmen, deren Märkte in keinerlei Zusammenhang stehen, durchgeführt werden. Die Abgrenzung zu Technologiediffusion ist daher fließend. Dies gilt auch für die Abgrenzung zum Wissenstransfer. Aufgrund der neueren Debatte in der Wissenstheorie zur Bedeutung impliziten Wissens wird inzwischen davon ausgegangen, dass zum Verständnis einer Technologie in erheblichem Umfang implizites Wissen erforderlich ist, das personengebundenes Wissen und Know-how umfasst, welches nicht oder nur schwer in eine kodifizierte Form zu bringen und deshalb nur schwer transferierbar ist. ${ }^{8}$ Das Verständnis von Technologietransfer hat sich auch wesentlich durch den Übergang vom linearen Modell des Innovationsprozesses auf rekursive, systemische Innovationsmodelle gewandelt. $\mathrm{Da}$ sich inzwischen die Erkenntnis durchgesetzt hat, dass es keine sequenzielle Abfolge der verschiedenen Phasen des Innovationsprozesses (Grundlagenforschung, angewandte Forschung, Entwicklung, Innovation) gibt, sondern diese rekursiv miteinander vernetzt sind, ist die Bedeutung längerfristiger, wechselseitiger Interaktion zwischen den Beteiligten in der Technologietransferförderung in den Vordergrund getreten. ${ }^{9}$

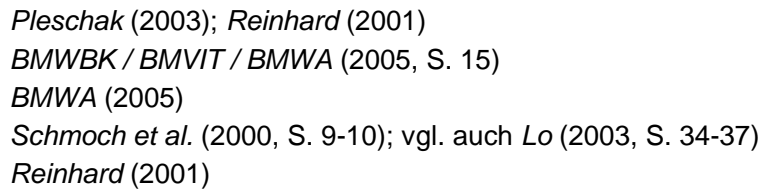


In der Evaluierung einer vorangegangenen Maßnahme zur Förderung des Technologietransfers (ITF-Schwerpunktprogramm Technologietransfer) wird folgende Definition von Technologietransfer verwendet, die diesen Erkenntnissen Rechnung trägt und daher auch der vorliegenden Evaluierung zugrunde gelegt wird:

"Technologietransfer beinhaltet die Übertragung von technologischem Wissen im weiteren Sinne, von einem Anwendungskontext in den anderen. Hierbei ist es unerheblich, welche Form das technologische Wissen besitzt und mit welchen Übertragungsinstrumenten es transferiert wird. Wesentlich ist, dass der Transfer einen vermehrten Technologiebestand des Technologieempfängers zur Folge hat. Unter technologischem Wissen wird das gesamte Spektrum von wissenschaftlichen Erkenntnissen bis hin zu kommerziell verwertbaren Technologien verstanden". ${ }^{10}$

Beim Transfer von Wissen im Gegensatz zur Information wird immer auch neues Wissen geschaffen, weil es in den neuen Erfahrungs- und Anwendungskontext verankert wird. Damit ist intelligenter Technologietransfer auch immer mit innovativer Weiterentwicklung des enthaltenen Wissens verbunden. Da beim Transfer von Technologie praktisch immer auch ein Transfer von implizitem Wissen erforderlich ist, sind grundsätzlich alle Transferformen besonders wirksam, die einen direkten Kontakt zwischen Transfergeber und -nehmer beinhalten, wie z.B. in der Kooperationsforschung. ${ }^{11}$ Das Förderprogramm protec $2002+$ zielt in seiner Ausrichtung auf eine Unterstützung genau dieser direkten Transferformen ab, die kontinuierliche, rekursive Interaktion zwischen den Akteuren im Innovationsprozess unterstützen. Als zentrale Hemmnisse für den Technologietransfer im KMU-Bereich können folgende Barrieren identifiziert werden: ${ }^{12}$

- Fehlendes Bewusstsein der KMU für ihre Bedürfnisse hinsichtlich neuer Technologien bzw. fehlende Kenntnis entsprechender Technologien;

- Identifizierung entsprechender Technologiegeber;

- Geringe Kooperationsbereitschaft und fehlende Kompetenzen sowie Kapazitäten für Vernetzung;

- Fehlen von Kapazitäten für langfristige Planung über das Altagsgeschäft hinaus, die dazu führen, dass im Gegensatz zu größeren Unternehmen häufig keine Innovationsstrategie entwickelt wird;

- Überwindung unterschiedlicher Kulturen, die Kommunikation, Verständnis und Vertrauensbildung entgegenstehen, z.B. in der Interaktion von KMU und Hochschulen.

Das Förderprogramm protec 2002+ ist darauf ausgerichtet, diese Schwierigkeiten zu verringern. Als Unterziele der Fördermaßnahme werden dementsprechend die folgenden Punkte genannt: ${ }^{13}$

- Verbesserung der Nutzung externer Ressourcen für KMU, insbesondere die Verbesserung des Zuganges zur Expertise in Forschungseinrichtungen (Universitäten, außeruniversitäre Forschungseinrichtungen, Fachhochschulen) zur Verbesserung des qualitativen Outputs der Unternehmen;

- Verbesserung des unternehmensinternen Innovationsmanagements;

- Verbesserung von Transferprozessen durch die Etablierung und Erprobung innovativer Transfermodelle (Kooperationsmodelle, Netzwerke und Plattformen) mit überregionaler Signalwirkung, die auf die Hebung des Innovationsniveaus von Unternehmen, Unternehmensgruppen und Sektoren (v. a. KMU) abzielen.

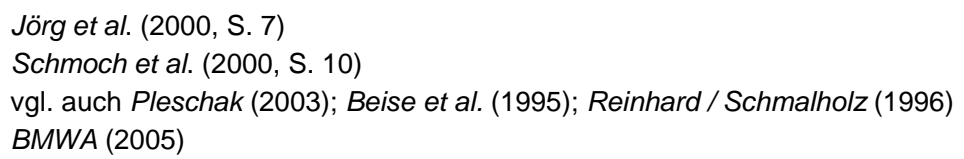


Der Programmansatz soll die Kooperationsbereitschaft und -fähigkeit zwischen den Akteuren des Innovationssystems auf nationaler und internationaler Ebene verbessern. ${ }^{14}$ Damit zielt das Programm nicht nur auf die Durchführung einzelner Projekte (Output), sondern darüber hinaus auf eine langfristige Verhaltensänderung der Zielgruppe (Impact), die sich nachhaltig in einer Verbesserung der Interaktion und Diffusion im österreichischen Innovationssystem auswirken soll. Im direkten Technologietransfer spielt neben der Technologiekompetenz auch die Kooperationskompetenz eine zentrale Rolle. Idealerweise weisen die beteiligten Unternehmen beide Kompetenzen bzw. Funktionen auf. Aufgrund der bekannten Kapazitäts- und Kompetenzengpässe bei KMU werden jedoch auch häufig Intermediäre (Berater, Technologiezentren) eingeschaltet, die keine Technologiefunktion besitzen, sondern nur Kooperationsfunktion. Eine Trennung dieser beiden Funktionen kann jedoch zu Effektivitätsverlusten im Technologietransfer führen, da die Problemorientierung fehlt. Für langfristigen Technologietransfer ist es daher notwendig, KMU dazu anzuregen und zu befähigen (enabling), dass sie beide Funktionen wahrnehmen können - sowohl die Technologie- als auch die Kooperationsfunktion. Aufgrund dieser doppelten Anforderung ist der Anspruch an das Innovations- und Kooperationsniveau der Projekte entsprechend zu relativieren, da die Lernfunktion, d.h. das Sammeln entsprechender Erfahrungen für die KMU, im Vordergrund steht.

\subsection{Bewertung der Ausgestaltung von protec 2002+}

\subsubsection{Ziele und Stärken des Programms}

Entsprechend der hohen Bedeutung des Technologietransfers für KMU und den genannten Schwierigkeiten wird das grundlegende Ziel einer staatlichen Förderung des Technologietransfers vor dem Hintergrund der kleinbetrieblichen Struktur Österreichs von den befragten ExpertInnen als außerordentlich wichtig bewertet. Technologietransfermechanismen werden als zentral für den Einstieg von KMU in neue Technologiefelder angesehen. Ein Experte hierzu:

"Viele KMU haben Technologie- und Wissenstransfer noch nicht als Ziel erkannt. Es gibt noch genügend Raum für entsprechende Initiativen. Die österreichische Industrie ist sehr kleinteilig strukturiert. Es ist ein harter Wettbewerb mit Unternehmen in mittel- und osteuropäischen Ländern in Gang gekommen. U. a. aus diesem Grunde muss das Technologieniveau insgesamt angehoben werden. Das gesamte Thema Technologietransfer muss den Unternehmen nahe gebracht werden. Kooperationsinitiativen und andere Maßnahmen sind extrem wichtig. Technologietransfer ist ein Muss in Österreich".

Die generelle Ausgestaltung von protec 2002+ bzgl. Ziel- und Schwerpunktsetzung sowie Zielgruppe wird von den befragten Personen fast ausnahmslos positiv bewertet. Ein Befragter beschreibt das Programm als "konkurrenzlos in der österreichischen Förderlandschaft". Sowohl von den befragten ExpertInnen als auch von den Fördernehmern wurde insgesamt eine hohe Zufriedenheit mit der Zielrichtung und prinzipiellen Ausgestaltung der Fördermaßnahme protec $2002+$ geäußert.

Mit der Zielgruppe eher kleiner und innovationsschwächerer Akteure, denen der Einstieg in verwandte und neue Technologiefelder ermöglicht werden soll, trifft das Programm den Bereich, der von den ExpertInnen als die wichtigste Aufgabe von Technologietransfer angesehen wird. Vor dem Hintergrund einer Stärkung der technologischen Leistungsfähigkeit sowie der Innovationskompetenz wird insbesondere der Adressatenkreis KMU als entscheidend bewertet. Dabei wurden mehrfach die Multiplikationseffekte und Vorbildfunktionen von erfolgreich an protec 2002+ teilnehmenden Unternehmen betont, die dazu beitragen die Scheu vor den Herausforderungen des Technologietransfers zu überwinden. Dass neben KMU auch wissenschaftliche Einrichtungen, Technologietransfer-Einrichtungen und Technologiezentren in der protec-Förderung eingebunden sind, wird positiv hervorgehoben (vergleiche hierzu auch die Analyse der protec-Projekte in Kapitel 4.2).

14 BMWA (2005) 
Die Förderung von Netzwerken bzw. des arbeitsteiligen Innovationsprozesses wird als wesentliche Stärke des Programms angesehen, insbesondere vor dem Hintergrund einer vielfach fehlenden Kooperationsbereitschaft kleiner und mittlerer Unternehmen insbesondere mit wissenschaftlichen Einrichtungen. Hier setzt protec 2002+, nach Aussagen der ExpertInnen, mit der Förderung vernetzten, strategischen und projektorientierten Denkens genau richtig an. Eine der Grundideen von protec 2002+ - nämlich die Herstellung oder Initiierung des Transfers zwischen Hochschule und Praxis - wird als gelungen bewertet. Positiv wird hervorgehoben, dass protec 2002+ auch in der Forschung Lerneffekte hervorruft, nämlich dass Unternehmen wichtige technologische Impulsgeber sein können. Der Praxisbezug des Programms wurde als eine weitere Stärke bezeichnet.

Bezüglich der grundlegenden Ausgestaltung von protec 2002+ als Projektförderung gaben die Befragten in der großen Mehrheit an, dass dies eine sinnvolle und - insbesondere im Kontext der Netzwerkorientierung - effiziente Form des Technologietransfers darstelle. Die finanziellen, von protec 2002+ ausgehenden Anreize wurden als sehr wichtig eingestuft. Betont wurde allerdings auch, dass dies nicht die einzige Form des Transfers darstellen würde, sondern immer in Kombination mit anderen Möglichkeiten Technologien und Wissen zu transferieren zu verstehen sei. Mehrfach hervorgehoben wurde die motivierende und verhaltensändernde Wirkung der Projektförderung in dem Sinne, dass Unternehmen gezwungen werden, sich mit den Anforderungen von Ausschreibungen auseinanderzusetzen, Projektideen und Zeitpläne zu entwickeln sowie Kooperationspartner zu suchen. Damit gibt protec 2002+ den Impuls zum Lernen eines strukturierten Projektvorgehens. Ein Experte und ein Fördernehmer hierzu:

"Es wird der Zwang zur systematischen Auseinandersetzung mit dem Thema Technologietransfer erzeugt - zum Beispiel indem ein kleiner Businessplan erstellt werden muss. Das strategische Element im Rahmen der Antragserstellung würde ich ganz hoch einstufen."

„Wenn wir ein neues Geschäftsfeld anfangen, dann steht die Langfristigkeit im Vordergrund. Wenn sie investieren streben sie irgendwann mal eine kommerzielle Umsetzung an. Eine andere Art von Förderung, z.B. einen Studenten oder Dissertanten, der für begrenze Zeit etwas macht, bringt uns ungleich weniger als im Falle einer langfristig angelegten Partnerschaft."

Von den Fördernehmern wurden zum einen die Vorteile eines direkten Technologietransfers "über Köpfe" angesprochen. Zum anderen wird der Anstoß zur Entwicklung langfristig angelegter Strukturen, Prozesse und Abläufe über den eigentlichen Förderzeitraum hinaus hervorgehoben:

"Wir sehen in diesem protec-TRANS Projekt eher die Initialunterstützung, die es uns ermöglicht hat, finanziell und natürlich auch unter Mitarbeit von unseren Partnern, das ganze erst einmal anzugehen. Und jetzt, nach Abschluss des Projekts, müssen wir mit eigenem Know-how und mit weiterer Zusammenarbeit mit den beiden, die Ergebnisse zu einem kommerziellen, nutzbaren Produkt machen." 
Die zentralen Stärken des Programms lassen sich wie folgt zusammenfassen:

- Bereits in der Beantragungsphase kann die Fördermaßnahme zu wesentlichen Erkenntnissen und Lerneffekten bei den beteiligten KMU führen, da sie sich mit ihren Innovationsbedürfnissen und -kompetenzen auseinandersetzen müssen. Die dafür notwendige systematische Bestandsaufnahme ihrer Kapazitäten, Fähigkeiten, Ressourcen, Schwächen und Lücken ist wesentliche Voraussetzung für ein zukünftiges aktives Innovationsmanagement, unabhängig von dem jeweils beantragten Förderprojekt.

- Die Programmausgestaltung gibt einen Anstoß zur Arbeitsteilung im Innovationsprozess auch für Kleinstunternehmen und bietet ihnen zugleich die Möglichkeit an einem größeren und eventuell interdisziplinären Projekt teilzunehmen. Durch die Einbindung in ein gemeinschaftliches Forschungsprojekt mit entsprechender eigener Investition in FuE-Vorhaben wird zum einen praktische Erfahrung in der Durchführung eigener FuE aufgebaut und weitergegeben, zum anderen übt die Zusammenarbeit Druck auf die Ergebniserzielung aus.

- Eine wichtige Stärke des Programms ist die Offenheit für unterschiedliche Akteursgruppen und die Unterstützung des Technologietransfers in heterogenen Akteurskonstellationen. Dabei ist der Neuigkeitsgrad naturgemäß insbesondere im Transfer zwischen Wissenschaft und Wirtschaft hoch, wenn Erkenntnisse aus der Grundlagenforschung erstmalig in den Anwendungsbereich transferiert werden. Hier entstehen radikale Innovationen mit potenziell hohem Technologiesprung.

- $\quad$ Aufgrund der oben genannten Barrieren für KMU im Technologietransfer zählt die Einbindung von Intermediären als wichtige Stärke von protec 2002+. In ihrer Funktion als Mobilisierer und Multiplikatoren können sie entscheidend dazu beitragen, die Anstoßwirkungen der Fördermaßnahme zu erhöhen, und sind wesentliche Faktoren für die Zielgruppenerreichung.

- $\quad$ Die Ausgestaltung von protec 2002+ als Projektförderung trifft - im Zusammenspiel mit anderen Möglichkeiten des Technologietransfers - die Bedürfnisse der Zielgruppe. Aufgrund der Vielfältigkeit der Ausprägungen, die Technologietransfer haben kann, ist eine ganze Bandbreite von Instrumenten sinnvoll. Die Projektförderung von protec 2002+ füllt hierbei eine wichtige Bedürfnislücke der Zielgruppe.

- $\quad$ Eine wesentliche Stärke des Förderansatzes ist zudem die überregionale Ausrichtung des Programms sowie die Themenoffenheit mit der Möglichkeit der bottom-up Entwicklung neuer und bestehender Technologiefelder.

Von den befragten Fördernehmern wurde vor diesem Hintergrund nur ein Bereich genannt, der von der Programmausrichtung nicht abgedeckt wird: die Unterstützung bei der Markteinführung. Aufgrund der EU-Beihilferegelung ist es trotz des Fokus' auf Innovation und Marktumsetzbarkeit der Projektergebnisse nicht möglich, in protec 2002+ die Markteinführung neuer Produkte und Dienstleistungen zu fördern. Diese Problematik gilt nicht nur für protec $2002+$, sondern generell für Innovationsförderprogramme. Gerade kleine und Kleinstunternehmen stehen jedoch oft vor der Schwierigkeit nach gelungenem Abschluss eines Forschungsprojekts die kostenintensive Markteinführung zu bewältigen. Häufig fehlt Know-how zur Abschätzung der Marktchancen und des Marktzugang insbesondere auf internationalen Märkten.

Die schriftliche Kurzbefragung unter den österreichischen Gewerbe- und Handwerksbetrieben bestätigt, dass der Bedarf nach Unterstützung im Bereich Innovationsaktivitäten vor allem im Abschätzen von Marktchancen und der Markteinführung besteht (Abbildung 2). Fast die Hälfte der Befragten (46\%) im Gewerbe- und Handwerksbereich sehen in diesem Bereich eigene Defizite. 
Abbildung 2: Bedarf nach Unterstützung von Innovationsaktivitäten (Mehrfachnennungen möglich), Anteil der Unternehmen in Prozent

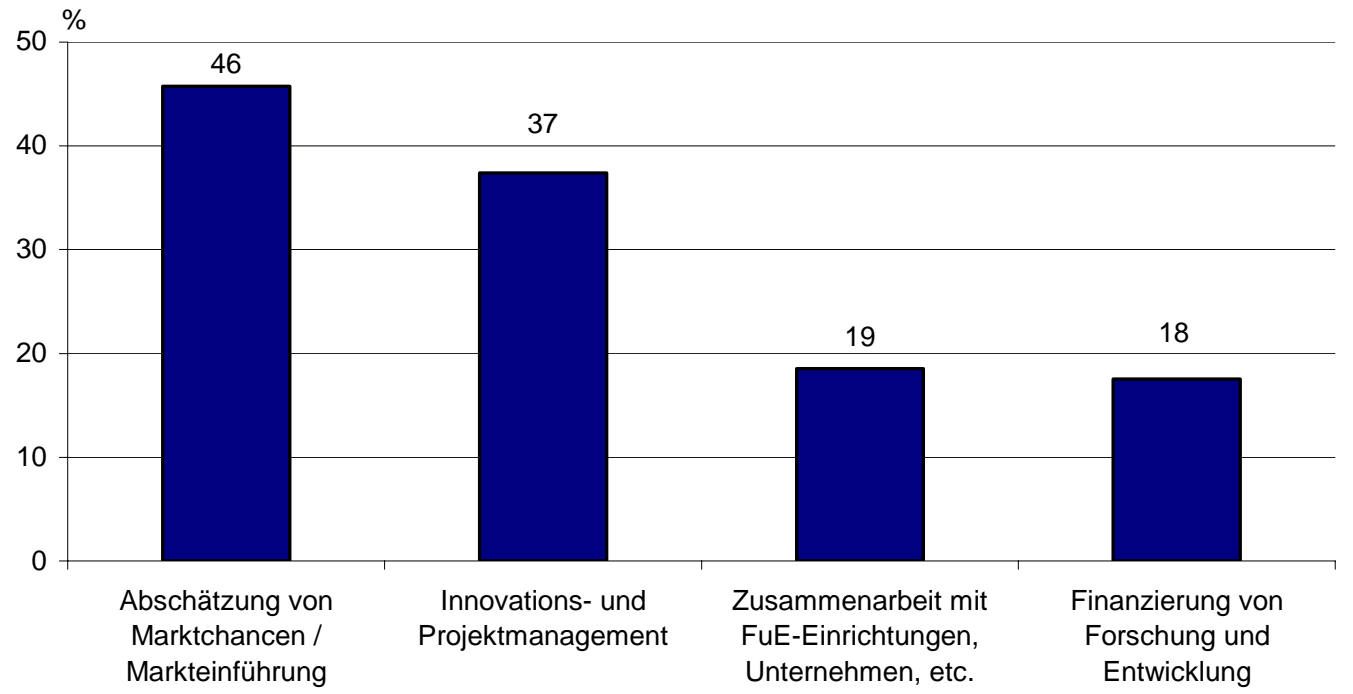

Quelle: Kurzbefragung Gewerbe- und Handwerksbetriebe, $N=600$ (nur Betriebe mit Unterstützungsbedarf)

Unterstützungsbedarf wird zudem von $37 \%$ der Betriebe im Bereich des Innovations- und Projektmanagement angemerkt. Die im Vergleich dazu relativ niedrige Zahl von Nennungen hinsichtlich eines Bedarfs im Bereich Zusammenarbeit mit FuE-Einrichtungen, Unternehmen, etc. (19\%) sowie der Finanzierung von Forschung und Entwicklung (18\%) lässt sich vermutlich darauf zurückführen, dass es sich bei der Befragtengruppe um Branchen handelt, die tendenziell durch eine geringe Forschungsintensität charakterisiert sind und deren Forschung eine geringe Kostenintensität aufweist. Zum Teil werden diese Schwächen vermutlich von den Unternehmen auch nicht wahrgenommen, obgleich sie bestehen. Kleine Unternehmen verfügen häufig nur über begrenzte Möglichkeiten, eigene Schwächen und Bedürfnisse hinsichtlich einer Weiterentwicklung des Unternehmens zu reflektieren.

Die Zielrichtung der Fördermaßnahme protec 2002+ wird ganz entscheidend durch die Schwerpunktsetzung in den drei Programmlinien protec-INNO, protec-NETplus und protec-TRANS bestimmt. Ein wesentliches Ziel der Zwischenevaluierung war daher, zu überprüfen

- ob diese Schwerpunktsetzung und die Ausgestaltung in den drei Förderlinien den Ansprüchen und Bedürfnissen der Zielgruppe entsprechen,

- welche Anreizwirkungen davon ausgehen und

- inwieweit sie dazu beitragen, Hemmnisse der Zielgruppe im FuE- und Innovationsbereich zu verringern.

Die drei Programmlinien innerhalb des Überbaus protec 2002+ wurden konzipiert, um nicht drei kleinteilige Programme zu schaffen, sondern die projektorientierte Technologietransferförderung in einer Maßnahme zu bündeln. Laut den Programmverantwortlichen im BMWA stand bei der Entwicklung der protec-INNO Linie vor allem die Förderung der Diffusion im Vordergrund. Damit sollte einer der zentralen Punkte des ausgelaufenen FINT-Programms weitergeführt werden. Die Programmlinie protec-NETplus wurde entwickelt, um den Hinweisen von Studien zur mangelnden Kooperation von KMU Rechnung zu tragen. Durch die Unterstützung der Zusammenführung wird auf positive Zusatzeffekte der Förderlinie im Innovationsprozess gesetzt. Der Programmschwerpunkt protec-TRANS ist von der Konzeption her stark an das ITF-Schwerpunktprogramm Technologietransfer angelehnt. Mit dieser Linie sollen KMU dazu motiviert werden, Technologietransferprojekte aus eigener Initiative heraus zu initiieren. 
Der überwiegenden Mehrheit der befragten Fördernehmer waren die drei Programmlinien im Großen und Ganzen bekannt. Über Detailkenntnisse verfügten allerdings meist nur die ProjektleiterInnen. Die Bereitschaft zur Übernahme einer Projektleitungsfunktion bzw. die Mobilisierung möglicher Partner für eine Projektidee geht logischerweise einher mit einer detaillierten Durchdringung der Programmrichtlinien. Institutionen und Organisationen, die lediglich als Konsortialpartner ohne Leitungsfunktion aktiv waren, sind über die genauere Ausgestaltung der Schwerpunkte häufig nicht informiert. In einigen wenigen Fällen war nur die eigene Programmlinie bekannt. Aus Sicht der Fördernehmer spielte die Aufteilung in Programmlinien nur eine geringe Rolle für ihren Zufriedenheitsgrad mit protec 2002+. Hier ist allerdings zu bedenken, dass es sich bei den Befragten durchwegs um erfolgreiche Fördernehmer handelt.

Kritischer wurde die Aufteilung von protec 2002+ in drei Programmlinien hingegen aus ExpertInnensicht gesehen. Hierbei wurde weniger die Zielrichtung der Linien in Frage gestellt als vielmehr die Schwierigkeit für KMU, sich angesichts der immer ausgefeilteren Förderlandschaft zu orientieren. Die damit einhergehende Erhöhung der Informationskosten verringert insgesamt gesehen den Nutzen, den KMU aus einer nur möglicherweise erfolgreichen Antragstellung ziehen, und führt damit zu unerwünschten Barrieren. Mit der Zahl unterschiedlicher Programme nimmt auch die Bedeutung von Informationsbrokern zu, die sich z.T. als Intermediäre an protec $2000+$ beteiligen. Von einigen ExpertInnen wurde daher die Schwierigkeit dieser Balance angesprochen: Intermediäre sind notwendig, um die KMU an eine (vom Gegenstand her komplexe) Antragstellung in einer vielteiligen Förderlandschaft heranzuführen, gleichzeitig kann eine zu hohe Intermediärsbeteiligung jedoch auch dazu führen, dass die primäre Zielgruppe der KMU in den Hintergrund tritt und die Förderung an Effektivität verliert. Angesichts der Vielzahl an technologie- und innovationspolitischen Programmen (siehe Kapitel 3.3) wurde zudem von einer ganzen Reihe von ExpertInnen - nicht zuletzt auch unter Hinweis auf das Volumen von protec 2000+ - darauf hingewiesen, dass die Aufteilung in drei Programmschwerpunkte die Sichtbarmachung des Programms nach außen und damit die Zugänglichkeit für die Zielgruppe KMU erschwert.

\subsubsection{Programmschwerpunkt protec-INNO}

Gegenstand von protec-INNO sind Projekte, die die Entwicklung, Diffusion und Implementierung von geeigneten Innovationsmanagement-Instrumenten sowie die Entwicklung, Erprobung und Anwendung neuer Beratungsansätze und -mechanismen zum Gegenstand haben. Ferner werden die Entwicklung und Verbreitung von Tools und "Good Practice" Modellen zur Unterstützung der Beschleunigung und Professionalisierung der ökonomischen Verwertung geistigen Eigentums gefördert. Im Monitoringbericht 3 wird zur Programmlinie protec-INNO die Einschätzung der Jury vermerkt, dass die eingereichten Projekte der dritten Ausschreibungsrunde relativ wenig neue Ansätze gezeigt hätten. Dies wirft die Frage auf, inwieweit die Entwicklung und Validierung neuer Innovationsmanagement-Tools in der von protec-INNO angesprochenen Form nach wie vor mit hoher Förderpriorität in einer eigenen Linie behandelt werden sollte. Die Herausforderung scheint in Zukunft eher in der Entwicklung von neuen Ansätzen für die Anwendung und Diffusion bereits existierender Instrumente zu bestehen (siehe auch Kapitel 4).

Aus Sicht der Fördernehmer stellt die Programmlinie protec-INNO von der Zielsetzung und Konzeption ein gutes Angebot dar, das es in der österreichischen Förderkulisse vorher in dieser Form nicht gegeben hat. Entsprechend der Ausgestaltung der Programmlinie handelt es sich dabei überwiegend um Beratungsdienstleister und Intermediäre, die InnovationsmanagementTools für die Zielgruppe KMU entwickeln. Neben dem Netzwerkgedanken und dem Anstoß eines Lernprozesses unter den Konsortialpartnern wird dementsprechend die in protec-INNO manifestierte Offenheit für neue Beratungsansätze und Möglichkeit der Methodenentwicklung hervorgehoben. Einer der Berater führt hierzu aus:

"Bezüglich der Förderung einer methodischen Weiterentwicklung von Beratungsangeboten gibt es nichts Vergleichbares. Dies ist auch die zentrale Stärke der Programmlinie. Sonst werden ja eher technologische Entwicklungen gefördert. Protec-INNO war für uns ein geniales Angebot." 
Trotz der insgesamt großen Zufriedenheit mit der Programmlinie wurde auch eine Reihe von Herausforderungen angesprochen. So wurden mehrfach Schwierigkeiten in der Antragstellung erwähnt, die sich aus dem Fördergegenstand von protec-INNO ergeben. Die Fördernehmer verweisen auf die Probleme der Darstellung des Nutzens bzw. Mehrwerts einer Methodenentwicklung, der Vermittlung der Ergebnisse und der Wirkungen einer neuen Methode. In einzelnen Fällen ist es darüber hinaus zu Problemen bei der Identifizierung von geeigneten KMU für die Testphase der Methodenentwicklung gekommen (siehe auch Kapitel 4.2.1). Die Schwierigkeiten haben sich hierbei dadurch ergeben, dass die Ansprüche und Komplexitätsgrade der entwickelten Tools und die Innovationskompetenz der KMU nicht kompatibel waren. Hierbei wurde auch von Fördernehmerseite die Frage aufgeworfen, inwieweit eine wissenschaftlich-theoretische Weiterentwicklung von Konzepten für die Bedürfnisse von KMU notwendig ist, da häufig einfache Innovationsmanagement-Tools ausreichen.

Von den befragten ExpertInnen wurde der Fokus auf professionelle Beratungsunternehmen in protec-INNO kritisch bewertet. Zudem wurde die Frage aufgeworfen, ob der Schwerpunkt in der Technologietransferförderung auch zukünftig noch auf der Entwicklung weiterer neuer Innovationsmanagement-Tools liegen sollte. Die Programmlinie protec-INNO hat in diesem Bereich in den vergangenen Jahren wertvolle Pionierarbeit geleistet und wichtige Anstöße gegeben. Die Schwerpunktsetzung erscheint jedoch inzwischen ihre Aufgabe erfüllt zu haben. Hierzu äußerte sich ein Experte:

"Protec-INNO ist meines Erachtens ausgereizt. Es gibt viele Tools für Innovationsstrategien, und in Österreich hat man durch diese Förderungen erreicht, dass noch weitere Tools zustande kommen. Es gibt inzwischen eine so hohe Zahl von Werkzeugen und Tools, dass man die nicht wieder neu erfinden muss."

Die Verbreitung der „Tools" zur Entwicklung von Innovationsstrategien und die Beseitigung bzw. Minderung der in diesem Bereich vielfach zu beobachtenden strukturellen Schwächen bei KMU, war einer der wesentlichen Erfolgsfaktoren dieser Programmlinie. Es gibt jedoch Hinweise darauf, dass die Diffusion an die Zielgruppe KMU in den letzten beiden Jahren nachgelassen hat (z.B. Schwierigkeiten in der Gewinnung von KMU-Projektpartner). Der Dominanz von Beratungsunternehmen in protec-INNO wurde im Zuge der nunmehr vierten Ausschreibungsrunde mit der Einforderung einer höheren KMU-Beteiligung, die entsprechend durch höhere Firmenbeiträge dokumentiert werden müssen, begegnet.

\subsubsection{Programmschwerpunkt protec-NETplus}

Mit protec-NETplus werden Projekte gefördert, die eine Anhebung des Innovationsniveaus (neue Produkte, Verfahren und Produktionslinien sowie Marktneuheiten) von Unternehmen (v. a. KMU) im Wege von Kooperationsmodellen, Netzwerken und Plattformen zum Ziel haben. Besonderes Augenmerk wird auf Projekte gerichtet, die einen technologischen und innovativen Impulscharakter haben (Hebelwirkung). Im Monitoringbericht 3 wird hervorgehoben, dass protec-NETplus besondere Anforderungen an die Antragsteller stellt, da zum einen die Netzwerkarchitektur und die Qualität der Zusammenarbeit innovativ sein sollen und zum anderen gleichzeitig ambitionierte Innovationsansprüche an die Unternehmenspartner hinsichtlich der zu entwickelnden Produkte und Dienstleistungen gestellt werden. Dazu führt die Monitoringgruppe aus: "Die bisherigen Ausschreibungen haben gezeigt, wie schwierig es ist, beiden Ansprüchen gerecht zu werden. Insbesondere die Innovativität der Projektinhalte kommt nicht selten zu kurz." Nichtsdestotrotz fällt die Bewertung der dritten Ausschreibungsrunde durch die Jury von protec-NETplus insgesamt positiv aus. Die Jury empfiehlt die Fortführung der Programmlinie. 
Die im Rahmen der Evaluierung befragten Fördernehmer gaben ausnahmslos an, mit dem Programmschwerpunkt protec-NETplus in hohem Maße zufrieden zu sein. Dies bezieht sich auf die Zielsetzung und Zielgruppe, die Ausgestaltung, die Förderabwicklung durch die FFG und die Förderintensität. Die zentralen Bedürfnisse der Fördernehmer werden mit dieser Programmlinie deutlich getroffen, die strategische Zielsetzung von protec-NETplus ist nach wie vor gültig. Die Förderung eines Netzwerk- und Kooperationsaufbaus wird einhellig als eine der wichtigsten Formen des Technologietransfers charakterisiert. Folgendes Zitat des Geschäftsführers eines im Rahmen von protec-NETplus geförderten KMU steht für eine ganze Reihe weiterer positiver Bewertungen, die sowohl von Fördernehmern als auch von ExpertInnen kommen:

"Ich muss sagen, ich habe es eigentlich sehr gut gefunden. Neben den anderen zwei Programmschienen hat mich protec-NETplus vom Inhalt her am besten angesprochen. Von der Art, wie es aufgebaut war: Produkte, Netzwerkaufbau, Kooperationsaufbau. Das hat mich persönlich am besten angesprochen. Also ich hätte hier keine Verbesserungsanregungen."

Auch die Intermediäre, die ja einen beträchtlichen Anteil der Fördernehmer ausmachen, betonen ihre Zufriedenheit mit protec-NETplus. Da das Initiieren von Kooperationen sowie Kontaktanbahnungen - insbesondere auch vor dem Hintergrund der Kenntnisse und Erfahrungen auf dem Gebiet der Fördermöglichkeiten - zu den zentralen Aufgaben der befragten Technologieund Innovationszentren sowie der Cluster-Initiativen gehört, schätzen sie die zusätzlichen Möglichkeiten, die innen protec-NETplus in der Projektarbeit bietet. Damit integriert sich aus Sicht der befragten Fördernehmer die Programmlinie hervorragend in die österreichische Förderlandschaft. Vom überwiegenden Teil der ExpertInnen wird der Mehrwert im Bereich protec-NETplus als nach wie vor sehr hoch bewertet, da die Kooperationsbereitschaft zwischen $\mathrm{KMU}$ trotz vieler Anstrengungen immer noch als gering einzuschätzen ist.

Analog zur großen Zufriedenheit der befragten Fördernehmer mit protec-NET plus wurden keine wesentlichen Verbesserungsvorschläge bezüglich dieser Programmlinie benannt. Dies trifft sowohl auf die Fördernehmer als auch auf die ExpertInnen zu. Konsequenterweise haben sich alle Befragten für die Fortsetzung dieser Programmlinie ausgesprochen. Festzuhalten bleibt, dass die Verbindung von Netzwerk- und Innovationsaktivitäten zwar anspruchsvoll ist, sich aber angesichts des inzwischen anerkannten Nutzens von Innovationsnetzwerken ${ }^{15}$ gerade daraus ein wichtiger Förderbereich ableitet.

\subsubsection{Programmschwerpunkt protec-TRANS}

Protec-TRANS fördert Technologietransfer-Projekte, die zur Stärkung der Forschungs- und Entwicklungskapazität von KMU beitragen. Die Transferprojekte sollen FuE-Aktivitäten von KMU durch die Bereitstellung von externem Wissen und vorhandenen Technologien mit dem Ziel der Auslösung von substantiellen Produktinnovationen (Marktneuheiten) und Dienstleistungen unterstützen. Protec-TRANS ermöglicht somit einen strukturell und finanziell unterstützten Tecnologietransfer. Im Monitoringbericht 3 wird die Programmlinie protec-TRANS aus Sicht der abwickelnden Stelle als „Nischenprodukt" für "besondere Situationen“ bezeichnet. Auf Grund der besonderen Projektkontexte und Inhalte besteht ein erhöhter Beratungsbedarf für die Abwicklungsstelle in dieser Programmlinie. Auffallend ist der relativ hohe Anteil forschungsintensiver Kleinstunternehmen.

15 vgl. z. B. Koschatzky et al. (2001) 
Die befragten Fördernehmer haben den Schwerpunkt protec-TRANS durchwegs positiv hinsichtlich der Erfüllung ihrer Bedürfnisse bewertet. Für eine ganze Reihe der befragten Unternehmen wurde durch die Teilnahme an protec-TRANS zum ersten Mal die Zusammenarbeit mit einer Universität oder Forschungseinrichtung realisiert. Folgendes Zitat steht stellvertretend für eine ganze Reihe ähnlicher Äußerungen:

"Wir haben das erste Mal sehr konkrete Zusammenarbeiten mit einer Universität gesucht und im Sinne von protec-TRANS ist wirklich Wissen von der Uni an uns geflossen. In der Form ist es ein sehr wichtiges Instrument, um die Verbindung zwischen Theorie und Praxis herzustellen. Also so gesehen hat das gepasst. Ich muss ganz ehrlich sagen, protec 2002+ hat von den Kosten her eine sehr hohe Bedeutung. Ich als KMU habe protec 2002+ als gute Chance für eine Förderung gesehen und als relativ angenehme, unkomplizierte Zusammenarbeit empfunden."

Gelobt wurde ebenfalls die intensive Betreuung durch die Förderstelle bzw. die Spezialberatung vor Ort. Diese habe dazu beigetragen, dass man überhaupt auf die Fördermöglichkeit aufmerksam geworden ist und man sich im Rahmen der Antragstellung stärker auf die eigentlichen Inhalte konzentrieren konnte. Der Aspekt der Spezialberatung Vor-Ort wurde auch von einigen ExpertInnen thematisiert und in einen Zusammenhang zum Bekanntheitsgrad der Programmlinie sowie der Notwendigkeit einer "strategischen Intelligenz" Vor-Ort gebracht (siehe Kapitel 5.1). Hierunter wird die (finanzielle, personelle und organisatorische) Fähigkeit der Vor-Ort ansässigen Intermediäre zur Information über protec 2002+ sowie der programmlinienspezifischen Betreuung und Beratung potentieller Antragsteller verstanden. Da diese "Ankopplungskompetenz" in den österreichischen Bundesländern unterschiedlich ausgeprägt ist, genießt die Programmlinie protec-TRANS trotz kontinuierlicher Ausschreibung nur einen begrenzten Bekanntheitsgrad, die Anzahl der Anträge erscheint ausbaufähig. Zudem erscheint eine Vor-Ort Lotsenfunktion auch durch den Umstand gerechtfertigt, dass nur eine begrenzte Mobilisierung der Zielgruppe durch den offenen Aufruf ("Open Call") möglich ist.

\subsection{Einordnung in die österreichische Förderlandschaft}

\subsubsection{Bundesförderung}

Eine Bewertung des Förderprogramms protec 2002+ muss vor dem Hintergrund der Entwicklung der österreichischen Förderlandschaft erfolgen. Im Bereich Technologietransfer dominierten Ende der 1980er, Anfang der 1990er Jahre vor allem die Technologie-, Innovations- und Gründerzentren. Hierbei sind insbesondere die Steiermark, Salzburg und Oberösterreich zu nennen. Eine 1998 durchgeführte Evaluierung der Regionalen Innovationsförderung (RIF) hat jedoch gezeigt, dass die Technologiezentren nicht die in sie gesetzten Erwartungen erfüllen konnten. In den 1990er Jahren wurden verstärkt Förderprogramme eingesetzt, um Wissensund Technologietransfer zu unterstützen. Ein Hauptaugenmerk lag dabei auf der Kooperation zwischen Wissenschaft und Wirtschaft, der besseren Nutzung der wissenschaftlichen Forschungsbasis und einer raschen Verwertung neuer wissenschaftlicher Erkenntnisse für Innovationen. ${ }^{16}$ In der zweiten Hälfte der 1990er Jahre ist insbesondere das ITF-Schwerpunktprogramm Technologietransfer zu nennen. Aufgrund einiger Überschneidungen mit anderen Fördermöglichkeiten und -instrumenten wurde das Programm nicht fortgeführt. Es erfüllte dennoch eine zentrale Funktion, indem es die Bedeutung von Technologietransfer als spezifische Aufgabe der Politik sichtbar machte. ${ }^{17}$ 
Der zunehmende Fokus auf die Förderung von Kooperationen, Netzwerken und Clustern in der Forschungs-, Technologie- und Innovationspolitik lässt sich nicht nur in Österreich, sondern generell in den OECD-Staaten feststellen. ${ }^{18}$ Die Verbesserung der Beziehungen zwischen Universitäten, Forschungseinrichtungen und Wirtschaft gehört inzwischen zu den zentralen Zielen der österreichischen Innovationspolitik. ${ }^{19}$ Charakteristisch für diese Art von Maßnahmen ist, dass eine Förderung nur für Zusammenschlüsse von Akteuren (Unternehmen, Forschungseinrichtungen, Intermediäre) gewährt wird beziehungsweise für Projekte, die Kooperationen, Netzwerke oder Cluster vorantreiben. Kurzfristiges Ziel ist dabei die Bündelung von Forschungskapazitäten, mittelfristiges Ziel eine schnellere Umsetzung von Forschungsergebnissen in Innovationen in Unternehmen und langfristiges Ziel die Entwicklung einer dauerhaften Zusammenarbeit zwischen Wissenschaft und Wirtschaft. Merkmal dieser Form der Kooperationsförderung ist der Verzicht auf die Schaffung institutioneller Infrastrukturen. Der Zusammenhalt der Netzwerke soll stattdessen durch das gemeinsame Forschungsinteresse gesichert werden. ${ }^{20}$

Ein wichtiges Ergebnis einer aktuellen Studie zu Instrumenten der Technologieförderung ${ }^{21}$ ist, dass sich in der Innovationsförderung, d.h. auch im Bereich Technologietransfer, keine Lücken im Förderangebot feststellen lassen. Es wird im Gegenteil eine beachtliche Programmvielfalt festgestellt. Die Autoren vermuten daher eine zunehmende Konkurrenz der Programme untereinander, die sich nicht nur auf die finanziellen Mittel, sondern auch auf die Aufmerksamkeit bei den adressierten Zielgruppen bezieht. ${ }^{22}$ Vor dem Hintergrund der österreichischen Innovationspolitik mit Bezug zum Technologietransfer ist ein wichtiges Merkmal von protec 2002+ die Möglichkeit, Projekte in ganz unterschiedlichen Konstellationen (bedingt durch die drei Programmlinien und Beteiligung verschiedener Akteure) einreichen zu können. Das Programm bietet daher den FördernehmerInnen eine breite Möglichkeit, die Förderung komplementär zu ihren eigenen Bedürfnissen einzusetzen. Eine Positionierung von protec 2002+ in der österreichischen Technologieförderungslandschaft kann daher nicht trennscharf erfolgen, sondern erschließt sich über Ähnlichkeiten in „verwandten“ Programmen wie z. B. kein thematischer Fokus, die adressierten Zielgruppen oder ein relativ heterogenes Projektportfolio betreffend der Partnerkonstellationen:

a) Innovationsförderung: Basisprogramme der FFG

b) Technologietransferförderung: BRIDGE - Das Brückenschlagprogramm

c) Netzwerkförderung:

- CIR-CE Cooperation in Innovation and Research with Central and Eastern Europe,

- REGplus - Ein Modul der Regionalen Impulsförderung,

- Impulsprogramm FHplus,

- prokis Querschnittstechnologien - ein Pilot in Ergänzung zu prokis - Programm zur Förderung von Kompetenzaufbau, Innovation und Strukturverbesserung der Kooperativen Forschungseinrichtungen in Österreich

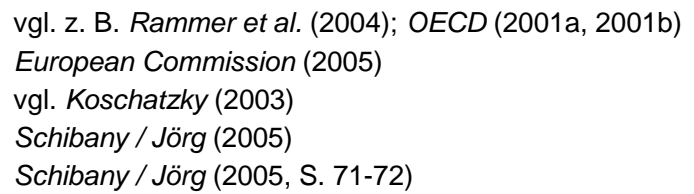


Im Unterschied zu diesen Fördermaßnahmen zeichnet sich protec 2002+ durch folgende Alleinstellungsmerkmale aus:

- Mit dem Kooperationsansatz werden mit protec 2002+ nach wie vor bestehende Hemmnisse der KMU in Bezug auf arbeitsteilige FuE-Aktivitäten angesprochen. Aufgrund der höheren Anforderungen, die Kooperationen an die beteiligten Akteure stellen, sind die Obergrenzen für Förderquoten in protec 2002+ höher als Z.B. bei den einzelbetrieblichen Basisprogrammen der FFG, die nicht direkt auf den Technologietransfer ausgerichtet sind.

- $\quad$ Explizites Ziel von protec 2002+ ist der Technologietransfer: darunter wird nicht nur der Technologietransfer Wissenschaft - Wirtschaft, sondern auch zwischen Unternehmen verstanden. Protec 2002+ ist damit deutlich anwendungsorientierter als z.B. der stärker grundlagenorientierte Technologietransfer, der im Rahmen von BRIDGE gefördert wird.

- $\quad$ Protec 2002+ fördert Technologietransfer ohne regionale Schwerpunkte - und damit auch bundesländerübergreifende Zusammenarbeit - in Österreich. Damit unterscheidet sich das Programm sowohl von länderspezifischen Maßnahmen als auch vom Förderprogramm CIR-CE, dessen Fokus auf Netzwerken mit Partnern aus Mittel- und Osteuropa liegt.

- Das Programm zielt auf eine spezifische Unterstützung der Zielgruppe KMU. Damit unterscheidet es sich von Förderprogrammen wie REGplus, FHplus oder prokis, bei denen zwar auch Netzwerke mit heterogenen Akteursstrukturen gefördert werden können, sich jedoch die primäre Zielgruppe unterscheidet (regionale Technologie-/ Innovationszentren, $\mathrm{FH}, \mathrm{ACR}$ ). Obgleich alle diese Fördermaßnahmen auf einen wesentlichen KMU-Nutzen zielen, ist protec 2002+ wesentlich stärker direkt auf diese Zielgruppe gerichtet und nicht im Umweg über die Intermediäre.

- Protec 2002+ fördert Technologietransferprojekte und keine Infrastrukturprojekte wie z.B. REGplus oder FHplus. Damit richtet sich das Förderprogramm auf die Unterstützung des direkten Technologietransfers in Form von innovationsorientierten Projekten, die auf interaktivem Austausch von Wissen und Technologien aufbauen. Dabei stehen Kompetenzaufbau, Kooperationserfahrung und die praktische Überwindung von Technologietransferbarrieren in konkreten Projekten im Vordergrund. Durch die Projekte müssen sich KMU mit ihren eigenen Bedürfnissen und Schwächen hinsichtlich der Technologieentwicklung auseinandersetzen, was eine längerfristige Verhaltensänderung hinsichtlich der Durchführung eigener und kooperativer FuE bewirken kann. Zudem ermöglicht die Zusammenarbeit das Entwickeln eines Verständnisses zwischen unterschiedlichen Akteuren (z.B. KMU, Forschungseinrichtung).

Nach Aussagen der befragten ExpertInnen stellt protec 2002+ damit eine "qualitativ wichtige Säule im österreichischen Technologietransfersystem" dar.

Eine gewisse Überschneidung zeigt sich in der Programmlinie protec-NETplus mit den Netzwerklinien der Programme REGplus und FHplus. Trotz unterschiedlicher Zielrichtungen entstehen zum Teil (in einzelnen Programmlinien) ähnliche Akteurskonstellationen - KMU-Netzwerke unter Einbindung regionaler Transfereinrichtungen oder Fachhochschulen - mit einem jeweils thematisch äußerst heterogenen Projektportfolio. Insgesamt sind die finanziellen Mittel, die in Österreich in technologie- und innovationspolitischen Programmen im Bereich Kooperation und Vernetzung eingesetzt werden, im internationalen Vergleich jedoch relativ niedrig. ${ }^{23}$ Die Vielfalt an Programmen heißt dementsprechend nicht, dass es eine zu hohe Förderung in dem Bereich gibt, aber möglicherweise eine zu kleinteilige (vgl. Tabelle 4).

23 Schibany / Jörg (2005) 
Tabelle 4: Vergleich ausgewählter österreichischer Netzwerk- und Kooperationsförderprogramme

\begin{tabular}{|c|c|c|c|c|c|c|}
\hline Programm & $\begin{array}{l}\text { erste } \\
\text { Aus- } \\
\text { schrei- } \\
\text { bung }\end{array}$ & Ziele des Programms & Zielgruppe & $\begin{array}{l}\text { Förderlinien I } \\
\text { Projektarten }\end{array}$ & $\begin{array}{c}\text { Zahl der geförderten } \\
\text { Projekte }\end{array}$ & Fördervolumen \\
\hline protec $2002+$ & 2002 & $\begin{array}{l}\text { Stärkung der } \\
\text { Innovationskraft und } \\
\text { Hebung der FuE- } \\
\text { Leistung der } \\
\text { Unternehmen in der } \\
\text { mittelständischen } \\
\text { Wirtschaft durch } \\
\text { Technologietransfer }\end{array}$ & KMU & $\begin{array}{l}\text { protec-INNO, } \\
\text { protec-NETplus, } \\
\text { protec-TRANS }\end{array}$ & $\begin{array}{l}\text { 2002/03: } 9 \text { INNO, } \\
5 \text { NETplus, } \\
\text { 2003/04: } 8 \text { INNO, } \\
6 \text { NETplus, } \\
\text { 2004/05: } 6 \text { INNO, } \\
\text { 15 NETplus, } \\
\text { seit 2003: } 21 \text { TRANS }\end{array}$ & $\begin{array}{l}\text { 2002/03: 2,1 Mio } € \text {, } \\
\text { 2003/04: 2,4 Mio } €, \\
\text { 2004/05: 3,3 Mio } €, \\
\text { TRANS: 2,5 Mio } € \\
\text { (per Juni 2005) }\end{array}$ \\
\hline REGplus & 2000 & $\begin{array}{l}\text { Erhöhung der } \\
\text { Leistungsfähigkeit der } \\
\text { Zentren, ihrer Aktivitäten } \\
\text { in der Region und für die } \\
\text { Unternehmen der } \\
\text { Region }\end{array}$ & Impulszentren & $\begin{array}{l}\text { Aufbauprojekte, } \\
\text { Erweiterungs- } \\
\text { projekte }\end{array}$ & $\begin{array}{l}\text { 2000: } 9 \text { Projekte, } \\
\text { 2003: } 15 \text { Projekte (7 } \\
\text { Aufbau, } 7 \text { Erweiterung, } \\
1 \text { Machbarkeitsunter- } \\
\text { suchung), } \\
\text { 2004: } 12 \text { Projekte }\end{array}$ & $\begin{array}{l}\text { 2003: 2,7 Mio € vom } \\
\text { Bund, 2,7 Mio € von } \\
\text { Ländern } \\
\text { 2004: 1,95 Mio €, } \\
\text { Kofinanzierung } \\
\text { durch Länder }\end{array}$ \\
\hline CIR-CE & 2005 & $\begin{array}{l}\text { Aufbau und Vertiefung } \\
\text { transnationaler } \\
\text { unternehmensge- } \\
\text { steuerter Innovations- } \\
\text { netzwerke (insbes. } \\
\text { KMU) zwischen } \\
\text { Österreich und Mittel-/ } \\
\text { Ost- und Südeuropa }\end{array}$ & $\begin{array}{l}\text { Unternehmen, } \\
\text { Intermediäre }\end{array}$ & $\begin{array}{l}\text { Innovationsprojekte, } \\
\text { Netzwerkprojekte, } \\
\text { Ausbildungs- } \\
\text { projekte }\end{array}$ & $\begin{array}{l}\text { 2005: } 3 \\
\text { Innovationsprojekte } \\
(0,8 \text { Mio. €), } 11 \\
\text { Netzwerkprojekte (1,3 } \\
\text { Mio. €), } 1 \\
\text { Ausbildungsprojekt } \\
(0,05 \text { Mio. } €)\end{array}$ & 2005: 2 Mio $€$ \\
\hline FHplus & 2002 & $\begin{array}{l}\text { Aufbau und Erhöhung } \\
\text { der FUE-Kapazität und - } \\
\text { Kompetenz an } \\
\text { Fachhochschulen und } \\
\text { FH-Studiengängen }\end{array}$ & $\begin{array}{l}\text { Fachhoch- } \\
\text { schulen }\end{array}$ & $\begin{array}{l}\text { Strukturaufbau- } \\
\text { vorhaben, } \\
\text { Kooperationsvor- } \\
\text { haben }\end{array}$ & $\begin{array}{l}\text { 2002: } 8 \\
\text { Kooperationsvorhaben } \\
\text { von } 20 \text { genehmigten } \\
\text { Projekten, } \\
2004: 14 \\
\text { Kooperationsvorhaben } \\
\text { von } 23 \text { genehmigten } \\
\text { Projekten }\end{array}$ & 2002: 10,6 Mio €, \\
\hline prokis & $\begin{array}{l}2003 / \\
2004 \\
2005\end{array}$ & $\begin{array}{l}\text { Verbesserung des } \\
\text { Dienstleistungs- } \\
\text { angebotes und Stärkung } \\
\text { der Kompetenz der } \\
\text { österreichischen } \\
\text { Kooperativen } \\
\text { Forschungseinrichtun- } \\
\text { gen als Partner der } \\
\text { Wirtschaft, insbesondere } \\
\text { KMU }\end{array}$ & $\begin{array}{l}\text { Österr. } \\
\text { Kooperative } \\
\text { Forschungs- } \\
\text { einrichtungen } \\
\text { ACR }\end{array}$ & \begin{tabular}{|l} 
prokis $^{04}$ \\
\\
Prokis-Querschnitts- \\
technologien \\
(Pilotprogramm zur \\
Verstärkung der \\
Netzwerkaktivitäten)
\end{tabular} & $\begin{array}{l}\text { 2003/2004: } \\
\text { 14 Projekte } \\
\text { 2005: } 4 \text { Projekte }\end{array}$ & $\begin{array}{l}\text { 2003/2004: } \\
\text { 18,3 Mio € } \\
\text { 2005: 0,5 Mio € }\end{array}$ \\
\hline BRIDGE & 2005 & $\begin{array}{l}\text { Gemeinsame Weiter- } \\
\text { entwicklung der } \\
\text { Potenziale der } \\
\text { Grundlagenforschung } \\
\text { und angewandten } \\
\text { Forschung }\end{array}$ & $\begin{array}{l}\text { Forschungs- } \\
\text { institute, } \\
\text { Unternehmen }\end{array}$ & Brücke 1, Brücke 2 & $\begin{array}{l}\text { 2. Ausschreibung } \\
\text { 2005: } 42 \text { Projekte }\end{array}$ & $\begin{array}{l}\text { 2. Ausschreibung } \\
\text { 2005: 13,8 Mio } €\end{array}$ \\
\hline
\end{tabular}

Protec 2002+ wird als "wertvolle, gute und stimmige Aktivität" in der Förderlandschaft des österreichischen Innovationssystems mit seinen spezifischen Anforderungen bewertet. Da der Bereich Technologietransfer aufgrund seiner Vielfalt ein ausdifferenziertes Förderinstrumentarium notwendig macht, deckt protec 2002+ eine Lücke ab. Für den Technologietransfer im vorwettbewerblichen Bereich füllt protec 2002+ nach ExpertInnenaussagen die Lücke zwischen der Forschung/Entwicklung und dem Endprodukt. Über die Einbindung von Technologiezentren und Hochschulen fungiert das Programm auch in der Mobilisierung vorhandener Infrastrukturen. 
Das etablierte Portfolio in Österreich weist einen starken Fokus auf eine breitenwirksame "Grundversorgung" aus, zusammengesetzt aus indirekter Förderung und einzelbetrieblicher bottom-up Projektförderung. Beide Förderansätze zeichnen sich durch die Gefahr relativ hoher Mitnahmeeffekte aus, die durch den weitgehenden Verzicht auf zielgruppenspezifische Selektionsmechanismen verstärkt wird ${ }^{24} \mathrm{Im}$ Vergleich dazu ist die finanzielle Ausstattung in der verhaltenssteuernden Innovationsförderung, d.h. in den Förderinstrumenten, die stärker auf eine bestimmte Verhaltensänderung der Fördernehmer wie z.B. die Durchführung von FuE in Kooperationen abzielen, in Österreich eher gering ausgeprägt. Dementsprechend kommt auch kleineren Fördermaßnahmen wie protec 2002+ ein entscheidendes Gewicht zu.

\subsubsection{Technologietransferförderung auf der Ebene der Bundesländer}

Die Alleinstellungsmerkmale von protec 2002+ und die angesprochene Förderlücke in der österreichischen Förderlandschaft werden auch beim Betrachten der Förderkulisse auf der Ebene der Bundesländer deutlich. Zwar gibt es auf regionaler Ebene kleinere Technologietransferprogramme - z.B. das KWF-Programm "Technologietransfer" in Kärnten - jedoch sind diese von der Förderhöhe und Laufzeit nicht oder nur bedingt mit protec 2002+ zu vergleichen. In einigen Bundesländern wird die Zielprojektion "Stimulierung des Technologietransfers" im weiteren Sinne zwar im Kontext der jeweiligen einzelbetrieblichen FuE-Förderprogramme der Bundesländer formuliert (so. z.B. im "Wirtschaftsimpulsprogramm" (WIP) Oberösterreichs oder im Rahmen der "Wirtschaftsbezogenen Innovations- und Forschungsförderung" des Landes Salzburg), jedoch handelt es sich hierbei meist um Anschlussförderungen an FuE-Förderungen des Bundes. Zu den auf Technologietransfer im weiteren Sinne zielenden Maßnahmen gehören z.B. allgemeine Beratungsdienstleistungen bezüglich des Erwerbs von Patentrechten und Lizenzen, Zukauf von Know-how und nicht patentierten technischen Kenntnissen, etc. Eine eigenständige FuE-Förderung - mit einzelnen Elementen der Unterstützung des Technologietransfers - praktizieren nur die Bundesländer Salzburg und Steiermark.

Im Gegensatz zur projektorientierten Transferförderung wird der Technologie- und Wissenstransfer in zahlreichen Bundesländern durch "Innovationsassistenten-Förderprogramme" realisiert. Mit dem Ziel eines Wissenstransfers "über Köpfe" beinhalten die Landesförderungen in der Regel Zuschüsse zu den Personalkosten der/des InnovationsassistentenIn (einschl. Personalnebenkosten). Gefördert werden $50 \%$ der Personalkosten im ersten Jahr und $25 \% \mathrm{im}$ zweiten Jahr (so beispielsweise in Oberösterreich). Darüber hinaus beinhalten einige InnovationsassistentInnen-Förderprogramme als ergänzendes Modul die Übernahme von Beratungskosten externer Dienstleister. Damit sollen KMU vor allem im Bereich der Entwicklung von Innovationsstrategien und des Managements von Innovationen unterstützt werden.

Die an dieser Stelle nur knapp konturierte FuE- bzw. Technologietransferförderung auf der Ebene der Bundesländer zeigt, dass im österreichischen Fördersystem zu protec 2002+ keine nennenswerte Alternative (v. a. im Sinne der Überregionalität, Förderhöhe und Förderdauer) existiert. Angeführte Beispiele können lediglich als komplementäre Förderansätze begriffen werden. Dies bedeutet jedoch nicht, dass die Aktivitäten auf der Ebene der Bundesländer bzw. auf regionaler Ebene unwichtig sind. Im Gegenteil: räumliche Nähe stellt eines der wesentlichen Elemente eines regionalen Innovationssystems dar. Der nicht nur in Österreich zu beobachtende "Trend" des vermehrten Einsatzes von Fördermitteln, die auf die Schaffung lokaler und regionaler Innovationsnetzwerke, Infrastrukturen, Plattformen und Cluster abzielen und die das gegenseitige Lernen der am Innovationsprozess involvierten Akteure (wie Transfer- und Beratungseinrichtungen, Forschungsinstitutionen, KMU usw.) unterstützen sollen, zielt auf regionale Besonderheiten berücksichtigende Förderpraktiken ab. Obwohl als Bundesprogramm konzipiert, ist mit Blick auf protec 2002+ - und hier spielt die regionale Ebene eine entscheidende Rolle - auf die Notwendigkeit einer "strategischen Intelligenz" Vor-Ort hinzuweisen. Offensichtlich können jedoch die Wirtschaftskammern bzw. Technologie- und Marketinggesellschaften, die Wirtschaftsförderungsgesellschaften oder auch die Gründungs-

24 Schibany / Jörg (2005) 
und Impulszentren - die als erste Anlaufstellen für mögliche Fördernehmer infrage kommen aus finanziellen/personellen Gründen nicht in allen Bundesländern eine optimale Betreuung der KMU gewährleisten.

\subsection{Internationale Ausrichtung von protec 2002+}

Wenngleich internationaler Austausch kein explizites Ziel von protec 2002+ ist, soll angesichts der Bedeutung der Internationalisierung für die Wettbewerbsfähigkeit auch von KMU, hier die grenzüberschreitende Bedeutung des Förderprogramms kurz skizziert werden. Bisher hat protec 2002+ noch nicht in großem Maße dazu beigetragen, Internationalisierungsaktivitäten von KMU zu forcieren. Zentrale Fragen sind, welche Anforderungen sich aus der zunehmenden Signifikanz von Internationalisierung für die Weiterentwicklung des Programms ergeben und welche Unterstützung Unternehmen im Rahmen des Technologietransfers benötigen.

Das EU-Beihilferecht spielte nach Aussage der befragten Fördernehmer für sie selbst und die Konsortiumbildung keine Rolle, die Deminimis-Regel wird von den Befragten nicht als Hürde wahrgenommen. Hier ist jedoch zu beachten, dass bei den Befragten der "survivor bias" greift, es bleibt demnach unsicher, wie hoch die Quote an potenziellen Antragstellern ist, die aufgrund der Deminimis-Regel auf die Antragstellung verzichtet haben.

Nach drei Ausschreibungsrunden nehmen an den Programmlinien protec-INNO und protecNETplus insgesamt 13 ausländische Partner teil (von insgesamt 337). Bei den eingereichten Anträgen waren es 24 von insgesamt 934 . Wenig überraschend dominieren dabei deutsche Partner (9). Neben der räumlichen Nähe und der Größe der deutschen Volkswirtschaft ist dies vermutlich vor allem auf die Sprache zurückzuführen. Daneben gibt es Teilnehmer aus der Schweiz, Großbritannien und den USA

Eine verstärkte Möglichkeit der Internationalisierung im Rahmen von protec 2002+ wurde von einer ganzen Reihe Fördernehmer befürwortet:

"Es wäre durchaus zu überlegen, ob man nicht internationale Netzwerke stärker fördert. Ich glaube, dass wir in Österreich nicht nur ein Kooperationsdefizit haben, sondern ein ganz, ganz großes Internationalisierungsproblem. (...) Das hielte ich schon für eine überdenkenswerte Variante, dass auch ein nationales Förderprogramm dann noch etwas dazulegt oder etwas davon abhängig macht, dass ausländische Partner dabei sind. In der Wissensgesellschaft werden wir immer fokussierter und spezialisierter. Und je spezialisierter wir sind, desto internationaler müssen wir werden. Mir schiene es daher schon sehr sinnvoll, Internationalität in Förderprogrammen zu einem wichtigen Punkt zu machen."

Neben der Einbindung ausländischer Partner wurde der Wunsch nach Internationalisierungsaktivitäten vor allem mit dem Ziel des Marktzugangs in anderen Ländern verbunden. Der wesentliche Unterstützungsbedarf wird dabei in der Förderung der internationalen Partner gesehen. Dies überrascht, da zu vermuten gewesen wäre, dass die Identifikation geeigneter Partner im Ausland ein zentrales Hemmnis für den internationalen Technologietransfer ist. Die Aussagen der befragten Fördernehmer lassen jedoch vermuten, dass dort, wo ein internationaler Austausch erwünscht ist, der Partner schon bekannt ist und es daher im wesentlichen um die Frage geht, wie dieser Partner in das Projekt eingebunden werden kann. Aufgrund des Aufwands internationaler Kooperationen waren einige Fördernehmer jedoch etwas zurückhaltender und wiesen darauf hin, dass man zunächst die regionale Kompetenz aus- bzw. aufbauen sollte. Eine Unterstützung internationaler Aktivitäten sei daher nicht von Beginn des Projekts, sondern erst in einer späteren Phase sinnvoll. 
Beispiel für eine gezielte Förderung transnationaler Projekte: PRO INNO II (Deutschland)

Die Fördermaßnahme "Programm Innovationskompetenz mittelständische Unternehmen" (PRO INNO II) des deutschen Bundesministeriums für Wirtschaft und Technologie zielt auf eine stärkere Vernetzung kleiner und mittlerer Unternehmen (KMU) untereinander und mit Forschungsinstitutionen im Bereich von Forschung und Entwicklung (FuE). Diese Kooperationen sind jedoch kein Selbstzweck, sondern vielmehr Mittel, um die Wettbewerbsfähigkeit von KMU auf internationalen Märkten durch Erhöhung der Innovationskraft sicherzustellen. PRO INNO ist zu verstehen als Instrument zur Förderung eines nachfrageorientierten Technologietransfers an KMU. Zusammen mit ihren Vorgängermaßnahmen (FoKo, PRO INNO) zählt PRO INNO II aufgrund der hohen Nutzung durch die Zielgruppe zu den wenigen Mittelstandsprogrammen der Bundesregierung mit einer spürbaren Breitenwirkung. Durch die Förderung sollen das technologische Leistungsniveau und die Kompetenz der beteiligten KMU deutlich erhöht werden (z.B. durch Einstieg in FuE, neues Technologiegebiet, neue Technologiekombination, Systemlösungskompetenz) oder die Kooperationserfahrungen wesentlich erweitert werden (z. B. durch mehrere Partner oder ausländische Partner). ${ }^{25}$

Zur stärkeren Unterstützung transnationaler Kooperationen wurden in PRO INNO II eine erhöhte Förderobergrenze (+50.000 €) und spezifische Förderboni eingeführt. Förderberechtigt sind nach wie vor lediglich die beteiligten deutschen KMU. Sowohl für Projekte mit EUREKAStatus als auch für Kooperationen mit Partner(n) aus anderen EU-Ländern wird jedoch der Fördersatz jeweils um $10 \%$ erhöht bei einem maximalen Fördersatz von $50 \%$ für KMU. Auf den oder die ausländischen Partner müssen mindestens $25 \%$ der Projektkosten aller Partner entfallen, um als transnationales Projekt zu zählen. Durch diese Anreize stieg die Anzahl transnationaler Projekte deutlich an. War es bei PRO INNO noch jedes zehnte Projekt, das in transnationaler Kooperation durchgeführt wurde, so ist es bei PRO INNO II jetzt jedes siebte Vorhaben. Dabei erlangen Kooperationsprojekte mit Partnern in den EU-Mitgliedsländern aus West- wie Osteuropa eine herausragende Stellung.

Neben den günstigen Förderkonditionen für transnationale Vorhaben, die nach Einschätzung der meisten Intermediäre ganz eindeutig zu dieser Entwicklung beitrugen, ist die internationale Ausrichtung und Präsenz von KMU auf Auslandsmärkten generell deutlich gestiegen. Die Anreize innerhalb von PRO INNO II verstärken diesen Prozess. Solche Vorhaben werden gerade auch dazu genutzt, den Zugang zu Auslandsmärkten zu stärken. Dies trifft jedoch nicht auf viele Kleinst- und Kleinunternehmen zu, für die internationale Vorhaben keinen Weg zur Stärkung ihrer Innovationsfähigkeit darstellen, da der Aufwand (monetär wie nicht-monetär) zu hoch ist und sie eher ihre gewachsenen, regionalen Kontakte nutzen. So werden in PRO INNO II auch ganz überwiegend Kooperationen zwischen Partnern gefördert, die bereits in verschiedenen Feldern zusammenarbeiteten. Die ExpertInnen betonten, dass transnationale Vorhaben mit erheblichen Kosten verbunden sind, die die höhere Förderobergrenze und den Förderbonus rechtfertigen. 
Bereits jetzt können internationale Partner in Projekte im Rahmen von protec 2002+ eingebunden werden, allerdings erhalten sie bisher keine Förderung. Die Möglichkeiten einer direkten Förderung internationaler KMU und Intermediäre werden derzeit im Rahmen von ERA-SME ausgelotet. Die Programmlinie protec-NETplus ist seit 2004 an dem ERA-net zusammen mit anderen europäischen KMU-Kooperationsprogrammen beteiligt. Ziel von ERA-SME ist die Vernetzung nationaler und regionaler Kooperationsprogramme zwischen KMU und Forschungseinrichtungen. Der Nutzen von ERA-SME liegt im Austausch von Best Practices zwischen den beteiligten Programmen und Organisationen in Bezug auf Programmdesign und -management zur Verbesserung der Programmeffizienz und -effektivität. ERA-SME ist eine Form von Demonstrationsprojekt für die Entwicklung und Implementierung neuer Strategien für transnationale Kooperationen zwischen Programmen, um einen Mehrwert sowohl für die Zielgruppen als auch für die Fördergeber zu schaffen. Es soll eine operationale Plattform für Fördergeber und Programmmanager bilden, um nachhaltige Kooperation zwischen Programmen der EU-Mitgliedsstaaten zu ermöglichen. Der Großteil der europäischen Staaten hat spezifische Fördermaßnahmen entwickelt, um die charakteristischen Hemmnisse für Technologietransfer von KMU zu verringern. Diese Maßnahmen fokussieren zum Teil direkt auf den KMU, zum Teil auf den Hochschulen und Forschungseinrichtungen, jedoch immer mit dem Ziel, die Kooperation zwischen diesen beiden Akteursgruppen zu verbessern.

Ein wesentliches Pilotprojekt im Rahmen von ERA-SME ist der Ende 2005 beendete gemeinsame Call von sechs nationalen Netzwerkförderprogrammen zum Thema KMU-Netzwerke, an dem auch die Programmlinie protec-NETplus beteiligt war. Dabei handelte es sich um ein experimentelles Programm mit einer begrenzten Zahl an Projekten, die den Weg zur Organisation und Durchführung eines durchgehend transnationalen Programms im Bereich Forschungs-, Technologie- und Innovationskooperation zwischen KMU und Hochschulen bzw. Forschungseinrichtungen aufzeigen sollen. Beteiligt sind neben Österreich Dänemark, Belgien (Flandern), Deutschland und Schweden. Fördernehmer aus diesen Ländern werden nach ihren jeweiligen nationalen Programmen finanziert. Tabelle 5 zeigt einen Überblick über die beteiligten Programme ergänzt um das Kooperationsprogramm PRO INNO II und das Netzwerkprogramm NEMO aus Deutschland.

Mindestanforderung für eine Teilnahme an dem Joint Call Pilotprojekt ist die aktive Kooperation zwischen mindestens zwei Forschungseinrichtungen aus unterschiedlichen Ländern mit unterschiedlichem Forschungsansatz (z.B. Grundlagenforschung und angewandte Forschung) und vier KMU. Primäres Ziel des Pilotprojekts ist die Stärkung der Wettbewerbskapazität von KMU durch:

- Zugang zum Wissen europäischer Forschungseinrichtungen;

- Verstärkung der Kooperations- und Wettbewerbskompetenz von KMU in der Beteiligung in internationalen FuE-Netzwerken;

- Verbesserung der Kooperationsfähigkeit europäischer Forschungseinrichtungen mit $\mathrm{KMU}$;

- gegenseitiger Lernprozesse über nationale Grenzen hinaus.

Die Projekte müssen Aktivitäten für die Diffusion der Projektergebnisse über das Projektteam hinaus enthalten. Trotz - oder möglicherweise aufgrund - gezielter Informationsausstreuung liegt die österreichische Beteiligung über dem erhofften Wert. 
Tabelle 5: Übersicht ausgewählter Innovationsnetzwerk- und Innovationskooperationsprogramme anderer Länder

\begin{tabular}{|c|c|c|c|c|c|c|c|c|c|}
\hline Land & Programm & Laufzeit & $\begin{array}{l}\text { Ziele des } \\
\text { Programms }\end{array}$ & Förderfähig & Zielgruppe & $\begin{array}{l}\text { Gegenstand der } \\
\text { Förderung }\end{array}$ & $\begin{array}{l}\text { Phasen im } \\
\text { Innovations- } \\
\text { prozess }\end{array}$ & $\begin{array}{l}\text { Zahl der } \\
\text { geförderten } \\
\text { Projekte }\end{array}$ & $\begin{array}{l}\text { Förder- } \\
\text { volumen }\end{array}$ \\
\hline $\begin{array}{l}\text { Belgien } \\
\text { (Flandern) }\end{array}$ & TETRA Fonds & seit 2004 & $\begin{array}{l}\text { Förderung des Tech- } \\
\text { nologietransfers } \\
\text { zwischen HS und } \\
\text { KMU }\end{array}$ & HS & $\begin{array}{l}\text { KMU, soziale } \\
\text { Organisa- } \\
\text { tionen }\end{array}$ & $\begin{array}{l}\text { Einzel- oder } \\
\text { Verbundprojekte, } \\
\text { die von einer HS } \\
\text { geleitet werden }\end{array}$ & $\begin{array}{l}\text { Vorwettbewerbliche } \\
\text { Forschung, indus- } \\
\text { trielle Forschung, } \\
\text { Entwicklung/Pro- } \\
\text { totyp, Technologie- } \\
\text { diffusion }\end{array}$ & $\begin{array}{l}\text { 2004: } 23 \\
\text { geförderte } \\
\text { Projekte mit } \\
\text { 324 Partnern }\end{array}$ & $\begin{array}{l}€ 6 \text { Mio. per } \\
\text { annum }\end{array}$ \\
\hline Dänemark & $\begin{array}{l}\text { Innovation } \\
\text { Consortiums }\end{array}$ & seit 2003 & $\begin{array}{l}\text { Stärkung der Kooper- } \\
\text { ation zwischen } \\
\text { Unternehmen, öffent- } \\
\text { lichen FE und tech- } \\
\text { nischen DL, um neue } \\
\text { Technologieplatt- } \\
\text { formen zu schaffen }\end{array}$ & $\begin{array}{l}\text { FE, HS, } \\
\text { Techno- } \\
\text { logiedienst- } \\
\text { leister }\end{array}$ & $\begin{array}{l}\text { Unternehme } \\
\text { n (insbes. } \\
\text { KMU), } \\
\text { Technologie- } \\
\text { dienstleister }\end{array}$ & $\begin{array}{l}\text { Innovationsprojekte } \\
\text { mit mind. } 2 \text { Unter- } \\
\text { nehmen, } 1 \text { FE/HS, } 1 \\
\text { Technologiedienst- } \\
\text { leister (Aufgabe: } \\
\text { Diffusion) }\end{array}$ & $\begin{array}{l}\text { Entwicklung/Pro- } \\
\text { totyp, Technologie- } \\
\text { diffusion, Koopera- } \\
\text { tionsförderung }\end{array}$ & $\begin{array}{l}\text { 2005: } 3 \text { Inno- } \\
\text { vationskon- } \\
\text { sortien, 2004: } \\
5 \text { Innovations- } \\
\text { konsortien }\end{array}$ & $\begin{array}{l}\text { 2005: } \\
\text { € } 5 \text { Mio., } \\
\text { 2004: } \\
€ \text { 6,5 Mio. }\end{array}$ \\
\hline Deutschland & $\begin{array}{l}\text { Innovative } \\
\text { Netzwerke } \\
\text { (InnoNet) }\end{array}$ & 1999-2009 & $\begin{array}{l}\text { Stärkung der Innova- } \\
\text { tionskraft und Wettbe- } \\
\text { werbsfähigkeit von } \\
\text { KMU sowie Be- } \\
\text { schleunigung des } \\
\text { Weges zur Markt- } \\
\text { fähigkeit von FuE- } \\
\text { Ergebnissen, stärkere } \\
\text { Ausrichtung der FE } \\
\text { auf KMU }\end{array}$ & HS, FE & KMU & $\begin{array}{l}\text { Netzwerkprojekte } \\
\text { zwischen mind. } 2 \\
\text { FE und mind. } 4 \\
\text { KMU; indirekte Zu- } \\
\text { schüsse für For- } \\
\text { schungsvorhaben, } \\
\text { für die die KMU } \\
\text { Verwertungsrechte } \\
\text { haben; gefördert } \\
\text { werden nur die FE }\end{array}$ & $\begin{array}{l}\text { Von FuE-Phase bis } \\
\text { zum Markteintritt, } \\
\text { Kooperations- } \\
\text { förderung }\end{array}$ & \begin{tabular}{|l|} 
2005: von der \\
Jury wurden \\
30 Projekte \\
zur Förderung \\
vorgeschlagen
\end{tabular} & $\begin{array}{l}\text { 2005: } \\
€ \text { 17,5 Mio. }\end{array}$ \\
\hline
\end{tabular}




\begin{tabular}{|c|c|c|c|c|c|c|c|c|c|}
\hline Deutschland & $\begin{array}{l}\text { Netzwerk- } \\
\text { management- } \\
\text { Ost (NEMO) }\end{array}$ & $\begin{array}{l}2002-2005 \\
\text { (Modell- } \\
\text { projekte } \\
\text { bereits 1999) }\end{array}$ & \begin{tabular}{|l|} 
Bildung und Ent- \\
wicklung leistungs- \\
fähiger Netzwerke \\
für Forschung, \\
Produktion und \\
Vermarktung
\end{tabular} & $\begin{array}{l}\text { Netzwerk- } \\
\text { management }\end{array}$ & $\begin{array}{l}\text { KMU (Unter- } \\
\text { nehmen, HS, } \\
\text { FE) }\end{array}$ & \begin{tabular}{|l} 
Zuschüsse für \\
Netzwerkmanage- \\
ment innovativer \\
Netzwerkprojekte \\
von mind. $6 \mathrm{KMU}$ \\
vorrangig aus \\
Ostdeutschland
\end{tabular} & $\begin{array}{l}\text { Kooperations- } \\
\text { förderung }\end{array}$ & \begin{tabular}{|l|} 
2005: 29 \\
Netzwerke mit \\
246 KMU, 4 HS, \\
35 FE und 1 \\
Verband
\end{tabular} & $€ 6$ Mio. \\
\hline Deutschland & $\begin{array}{l}\text { Programm } \\
\text { Innovations- } \\
\text { kompetenz } \\
\text { mittelstän- } \\
\text { dische Unter- } \\
\text { nehmen (PRO } \\
\text { INNO II) }\end{array}$ & $\begin{array}{l}2004-2008 \\
\text { (in ähnl. } \\
\text { Form seit } \\
1993 \text { ) }\end{array}$ & \begin{tabular}{|l|} 
Verstärkung der \\
technologischen \\
Basis von KMU \\
und deren FuE- \\
Kooperationen mit \\
Unternehmen im \\
In- und Ausland
\end{tabular} & KMU, HS, FE & KMU, HS, FE & $\begin{array}{l}\text { Zuschüsse zu FuE- } \\
\text { Kooperations- } \\
\text { vorhaben zwischen } \\
\text { KMU und zwischen } \\
\text { KMU und HS/FE } \\
\text { sowie zu } \\
\text { Unteraufträgen und } \\
\text { Personalaustausch } \\
\text { von KMU an FE }\end{array}$ & $\begin{array}{l}\text { Von FuE-Phase } \\
\text { bis zum Markt- } \\
\text { eintritt, Koopera- } \\
\text { tionsförderung }\end{array}$ & $\begin{array}{l}\text { 2005: } 1.596 \\
\text { Akteure geför- } \\
\text { dert, davon } \\
1.255 \mathrm{KMU}, \\
\text { überwiegend } \\
\text { bilaterale } \\
\text { Kooperationen }\end{array}$ & $\begin{array}{l}\text { 2005: } \\
€ 156 \text { Mio. }\end{array}$ \\
\hline Schweden & $\begin{array}{l}\text { Active } \\
\text { Industrial } \\
\text { Collaboration }\end{array}$ & seit 1998 & \begin{tabular}{|l|} 
Stärkere Ausrich- \\
tung der For- \\
schung an Univer- \\
sitäten an den \\
Bedürfnissen der \\
Wirtschaft und \\
Verbesserung der \\
Zusammenarbeit, \\
Etablierung eines \\
aktiven Technolo- \\
gietransfers, Ver- \\
ringerung "kultu- \\
reller" Barrieren
\end{tabular} & $\begin{array}{l}\text { Unternehmen, } \\
\text { HS, FE }\end{array}$ & $\begin{array}{l}\text { Unternehmen, } \\
\text { HS, FE }\end{array}$ & $\begin{array}{l}\text { Netzwerkprojekte } \\
\text { zwischen mind. } 1 \\
\text { HS, mind. } 1 \text { FE, } \\
\text { mind. } 6 \text { Unterneh- } \\
\text { men davon mind. } 1 \\
\text { KMU }\end{array}$ & $\begin{array}{l}\text { Industrielle For- } \\
\text { schung, Entwick- } \\
\text { lung/Prototyp }\end{array}$ & \begin{tabular}{|l|} 
Pro Jahr werden \\
etwa 30 Pro- \\
jekte gefördert, \\
der Eigenanteil \\
der Unterneh- \\
men liegt bei \\
$50 \%$ (Eigen- \\
leistung)
\end{tabular} & $\begin{array}{l}€ 10 \text { Mio. } \\
\text { per annum }\end{array}$ \\
\hline
\end{tabular}

Quelle: KMU FORSCHUNG AUSTRIA 
Für die Fortführung von protec 2002+ werden derzeit darüber hinaus auch andere Finanzierungsquellen auf europäischer Ebene geprüft. Hierbei ist jedoch auch auf die Schwierigkeiten einer derartigen Öffnung des Programms hinzuweisen. Dazu gehört vor allem der Verlust an Eigensteuerung. Werden internationale Förderquellen angesprochen, so wird es nicht mehr im gleichen Maße wie jetzt möglich sein, eigene Ziele hinsichtlich der Zielgruppen und Programmgestaltung zu verfolgen, da die möglicherweise divergierenden Ziele der ausländischen Fördergeber zu berücksichtigen wären. Von einigen Fördernehmern wurde daher in diesem Zusammenhang darauf verwiesen, dass sie protec 2002+ in erster Linie als österreichische Förderung verstehen. Obgleich diese Position verständlich ist, erscheint eine verstärkte Berücksichtigung internationaler Austauschbeziehungen in dem Förderprogramm vor dem Hintergrund der allgemeinen Entwicklungen jedoch notwendig. Auch österreichische KMU befinden sich zunehmend im internationalen Wettbewerb und werden sowohl ausländische Wissenspartner als auch den Zugang auf internationale Märkte dringend benötigen, wenn sie in diesem Wettbewerb bestehen wollen. Gleichzeitig darf jedoch nicht vernachlässigt werden, dass es sich bei der Zielgruppe KMU um Akteure handelt, die häufig kaum über die notwendigen Kapazitäten und Kompetenzen verfügen, um nationale FuE-Kooperationen durchzuführen, und dementsprechend der Anspruch an Internationalisierung nicht zu hoch angesetzt werden darf.

Im Förderprogramm CIR-CE, dessen explizites Ziel es ist, die Internationalisierung von KMU voranzutreiben, wird daher eine Trennung in unterschiedliche Projekttypen mit unterschiedlichen Zielgruppen vorgenommen. Dahinter steht ein Stufenmodell der Internationalisierung. Den ersten Schritt stellen die so genannten Netzwerkprojekte dar, in denen Intermediäre (Cluster, Forschungskompetenzzentren, kooperative Forschungsinstitute, Technologieparks) unter Einbindung von KMU die Zusammenarbeit mit den nicht-österreichischen Partnern im Netzwerk organisieren. Erst in der nächsten Stufe, den Innovationsprojekten, in denen konkrete FuE-Projekte im Zentrum stehen, soll der Unternehmenssektor die steuernde Rolle übernehmen. Dieses Modell hat den Vorteil, dass KMU an internationale Zusammenarbeit schrittweise herangeführt werden, die Möglichkeit zum Kooperationslernen in den Netzwerkprojekten gegeben wird und die Intermediäre durch ihre größere Erfahrung zum Abbau von Barrieren beitragen können. Allerdings besteht auch die Gefahr, dass eine solche Fördermöglichkeit vor allem zu einer Internationalisierung der Intermediäre beiträgt und weniger der Zielgruppe KMU. Angesichts der inhaltlichen Nähe zu CIR-CE und dessen internationaler Ausrichtung ist für jede weitere Entwicklung im Bereich Internationalisierung eine intensive Absprache mit CIR-CE zu empfehlen. Die Erfahrungen von CIR-CE - insbesondere hinsichtlich des Erreichens nachhaltiger Kooperationseffekte bei den KMU - sollten beim zukünftigen Programmdesign berücksichtigt werden. 


\section{Analyse der Programmwirkungen}

\subsection{Zielgruppenerreichung und Struktur der Fördernehmer}

\subsubsection{Mobilisierungswirkungen des Programms}

Zielgruppe des Programms protec 2002+ für den Technologietransfer in direkten Kooperationen sind in erster Linie kleine und mittlere Unternehmen (KMU). Aufgrund der Schwierigkeiten, vor denen KMU in der Beantragung und Durchführung komplexerer Kooperationsprojekte stehen, werden zusätzlich Einrichtungen angesprochen, deren Know-how für KMU eine wichtige Rolle spielt bzw. die den gewünschten Technologietransfer unterstützen. Dies umfasst sowohl Forschungseinrichtungen als auch Multiplikatoren wie spezialisierte Technologietransfereinrichtungen und Unternehmensberater. Hinsichtlich der Branchen und Technologiesegmente steht das Programm allen Akteuren offen und ist demnach nach dem bottom-up Prinzip angelegt, in dem die Bedürfnisse der Zielgruppen im Vordergrund stehen und nicht die Steuerung durch den Fördergeber. Gleichzeitig stellt das Programm jedoch zum Teil hohe Ansprüche an die Vernetzungskompetenz, das Diffusionskonzept und den innovativen Gehalt der zu fördernden Projekte und Akteure. Bevor die Evaluation sich mit den Wirkungen des Programms in konkreten Projekten beschäftigt, wird daher im Folgenden zunächst die Mobilisierungswirkung analysiert, das heißt die Erreichung der Zielgruppe und damit auch die Breitenwirkung des Programms.

\section{Öffentlichkeitsarbeit im Rahmen von protec 2002+}

Für die Bekanntmachung des Programms protec 2002+ wurde auf ein breites Spektrum an Informationskanälen zurückgegriffen, um sowohl der Vielfalt des Themas Technologietransfer als auch der Heterogenität der Zielgruppe gerecht werden zu können. Im Zuge der ersten Ausschreibungsrunde wurde dabei neben der Versendung von Foldern und der Durchführung entsprechender E-Mailaussendungen auf Basis des Adressmaterials des BMWA, der Abwicklungsstellen sowie der Wirtschaftskammer Österreich, vor allem die direkte Kommunikation mit den Zielgruppen vor Ort gesucht. In diesem Zusammenhang wurde eine Veranstaltungsreihe absolviert bzw. relevante Veranstaltungen genutzt, um protec 2002+ direkt vor Ort zu kommunizieren. Insgesamt konnten dabei ca. 400 Personen angesprochen werden. Darüber hinaus wurden Artikel und Beiträge in diversen Fachmedien (z.B. Austria Innovativ, Kammer Nachrichten etc.) platziert und Informationsplattformen für die Bekanntmachung von protec 2002+ genutzt.

Die breite Öffentlichkeitsarbeit im Rahmen von protec 2002+ in der ersten Ausschreibungsrunde brachte ein vergleichsweise hohes Echo. Insgesamt wurden 55 Projekte für die beiden Programmlinien protec-INNO und protec-NETplus eingereicht, an denen sich insgesamt 390 Organisationen beteiligten (siehe Tabelle 6), davon 274 kleine und mittlere Unternehmen aus unterschiedlichen Branchen.

Im Lichte der Erfahrungen aus der ersten Ausschreibungsrunde wurde die Kommunikationsarbeit im Zuge der zweiten Ausschreibungsrunde noch weiter verstärkt und die Ausschreibungsunterlagen für protec-INNO und protec-NETplus adaptiert. Insbesondere die Einführung von sog. Proposers Days für die beiden Programmlinien protec-INNO und protec-NETplus zur Unterstützung der Antragsteller hat sich dabei als sehr zielführend herausgestellt. Insgesamt wurden bisher 6 Proposers Days abgehalten, auf denen den Teilnehmerlnnen die Ziele und Inhalte der jeweiligen Programmlinien, Detailinformationen zur Einreichung von Förderanträgen sowie zu den jeweiligen Förderkriterien und „Good Practice“ Beispiele erfolgreicher Projekte präsentiert wurden. Darüber hinaus boten die Proposers Days die Gelegenheit, Fragen bzgl. der Antragstellung an das Programmmanagement zu stellen. Das Angebot des Proposers Days wurde vielfach genutzt und von den Fördernehmern auch im Rahmen der durchgeführten Interviews als sehr positiv und hilfreich hervor gestrichen. 
Die Resonanz auf die zweite Ausschreibungsrunde war gemessen an der Zahl der Projektanträge mit insgesamt 37 eingereichten Projekten deutlich geringer als in der ersten Runde. Dies kann möglicherweise dadurch erklärt werden, dass zu Beginn eines Programms häufig bereits bestehende Projektideen eingereicht werden. Die vergleichsweise höhere Erfolgsquote in der zweiten Ausschreibungsrunde - durch die die Zahl der zur Förderung vorgeschlagenen Projekte mit insgesamt 14 auf dem Niveau der ersten Runde blieb - verdeutlicht dementsprechend auch, dass die in der zweiten Ausschreibungsrunde eingereichten Projekte stärker dem Ziel des Programms entsprochen haben und die Antragsqualität sich erhöht hat. Gleichzeitig ist auch die durchschnittliche Projektgröße in den beiden Programmlinien gestiegen (siehe Tabelle 6).

Tabelle 6: $\quad$ Förderanträge und ausgeschüttete Fördermittel nach Ausschreibungsrunde

\begin{tabular}{|c|c|c|c|c|}
\hline & \multicolumn{2}{|c|}{ protec-INNO } & \multicolumn{2}{|c|}{ protec-NETplus } \\
\hline & eingereicht & gefördert & eingereicht & gefördert \\
\hline 1. Ausschreibungsrunde & \multicolumn{2}{|c|}{ 15. Sept. bis 16. Dez. 2002} & \multicolumn{2}{|c|}{ 1. Sept. bis 29. Nov. 2002} \\
\hline Anzahl der Förderanträge & 28 & 9 & 27 & 5 \\
\hline teilnehmende Partner & 144 & 60 & 246 & 35 \\
\hline $\begin{array}{l}\text { Partner pro Netzwerk } \\
\text { (Durchschnitt) }\end{array}$ & 5,1 & 6,7 & 9,1 & 7 \\
\hline Projektvolumen in $€$ & 8.392 .266 & 2.727 .306 & 9.667 .586 & 1.940 .314 \\
\hline Fördermittel in $€$ & & 1.161 .944 & & 969.157 \\
\hline 2. Ausschreibungsrunde & \multicolumn{4}{|c|}{ 3. Nov. 2003 bis 30. Jan. 2004} \\
\hline Anzahl der Förderanträge & 20 & 8 & 17 & 6 \\
\hline teilnehmende Partner & 105 & 49 & 130 & 52 \\
\hline $\begin{array}{l}\text { Partner pro Netzwerk } \\
\text { (Durchschnitt) }\end{array}$ & 5,3 & 6,1 & 7,6 & 9 \\
\hline Projektvolumen in $€$ & 6.519 .082 & 2.567 .737 & 6.489 .646 & 2.688 .451 \\
\hline Fördermittel in $€$ & & 1.275 .168 & & 1.093 .396 \\
\hline 3. Ausschreibungsrunde & \multicolumn{4}{|c|}{ 2. Nov. 2004 bis 31. Jan. 2005} \\
\hline Anzahl der Förderanträge & 15 & 6 & 29 & 15 \\
\hline teilnehmende Partner & 117 & 44 & 192 & 97 \\
\hline $\begin{array}{l}\text { Partner pro Netzwerk } \\
\text { (Durchschnitt) }\end{array}$ & 7,8 & 7,3 & 6,6 & 6,5 \\
\hline Projektvolumen in $€$ & 4.945 .653 & 1.650 .756 & 13.471 .127 & 5.600 .643 \\
\hline Fördermittel in $€$ & & 815.752 & & 2.450 .065 \\
\hline \multicolumn{5}{|l|}{ Insgesamt } \\
\hline Anzahl der Förderanträge & 63 & 23 & 73 & 26 \\
\hline teilnehmende Partner & 366 & 153 & 568 & 184 \\
\hline Projektvolumen in $€$ & 19.857 .001 & 6.945 .799 & 29.628 .359 & 10.229 .408 \\
\hline Fördermittel in $€$ & & 3.252 .864 & & 4.512 .618 \\
\hline Quelle: protec $2002+$, Monitorin & cht 3 (2005) & & & \\
\hline
\end{tabular}


Im Zuge der dritten Ausschreibungsrunde wurde die Kommunikation des Programms über Presse- und E-Mailaussendungen, Informationsveranstaltungen sowie entsprechende Proposers Days fortgesetzt. Darüber hinaus erfolgte eine Adaption der Ausschreibungsunterlagen und Richtlinien. Dazu gehören u. a. eine Anpassung an die neue KMU-Definition ab 1.1.2005 ${ }^{26}$ sowie eine neue Darstellung von protec 2002+ auf der Homepage des BMWA und der Homepage der beiden Programmabwicklungsstellen.

Die dritte Ausschreibungsrunde hat mit insgesamt 44 eingereichten Projektanträgen eine stärkere Resonanz gefunden als die vorhergehende, insbesondere für die Programmlinie protec-NETplus wurden mit 29 Projektanträge nahezu doppelte so viele Projekte eingereicht wie in der zweiten Ausschreibungsrunde. Demgegenüber ist das Projektaufkommen bei protecINNO deutlich zurückgegangen (siehe Tabelle 6). Auch die durchschnittliche Projektgröße hat sich in beiden Programmlinien gegenüber der zweiten Ausschreibungsrunde wieder deutlich reduziert und liegt sowohl bei protec-INNO als auch bei protec-NETplus unter jener der ersten Ausschreibungsrunde. Dies ist jedoch in erster Linie darauf zurückzuführen, das die Jurys in der dritten Ausschreibungsrunde bei beiden Programmlinien tendenziell eher kleinere Projekte favorisiert haben; die durchschnittliche Projektgröße der geförderten Projekte liegt bei beiden Programmlinien deutlich unter jener der abgelehnten.

Insgesamt haben sich an den drei Ausschreibungen von protec-INNO und protec-NETplus 934 Organisationen und Unternehmen beteiligt, 337 (36\%) davon erfolgreich. Die erfolgreichen Teilnehmer haben 49 Projekte im Gesamtvolumen von $€ 17,2$ Mio eingereicht und $€ 7,7$ Mio Förderung zugesprochen bekommen. Die dritte Programmlinie protec-TRANS wurde im offenen Antragsprinzip abgewickelt (siehe Kapitel 5.2.2). Seit Programmstart am 7. April 2003 wurden per 30. Juni 2005 insgesamt 21 protec-TRANS Projekte mit einem Gesamtvolumen von $€ 5,2$ Mio und einer Fördersumme in Höhe von insgesamt € 2,17 Mio gefördert. In Summe waren in den 21 protec-TRANS Projekten 56 Teilnehmer involviert.

In Bezug auf die Öffentlichkeitsarbeit von protec 2002+ haben die Analysen gezeigt, dass vor allem die im Internet verfügbaren Programmausschreibungen und Informationsmöglichkeiten für den Großteil der befragten Institutionen und Unternehmen eine wichtige Rolle spielen, um sich über Fördermöglichkeiten zu informieren. Als wesentliche Informationsquellen wurden v. a. das Wirtschaftsministerium, die Wirtschaftskammern, Intermediäre sowie die FFG und der ERPFonds genannt. Die Informationsbereitstellung der entsprechenden Förderinstitutionen mittels E-Mail-Verteiler war dabei für eine ganze Reihe der befragten Fördernehmer ebenfalls bedeutend. Insgesamt fühle man sich von allen relevanten Einrichtungen rechtzeitig, ausreichend und gut informiert. Deutlich wurde jedoch auch, dass die meisten der geförderten Institutionen bzw. Unternehmen durchaus einen gewissen Erfahrungsschatz bezüglich Fördermöglichkeiten haben. Dies trifft tendenziell stärker auf Intermediäre sowie Beratungsunternehmen zu. In diesem Kontext wurde zudem das Vorhandsein persönlicher Kontakte in den entsprechenden Institutionen betont.

Bei den befragten KMU ist das Bild weniger einheitlich. Von einem regelmäßigen und systematischen "Screening" der Fördermöglichkeiten kann seitens der KMU nicht gesprochen werden. Zeitknappheit und eine große Arbeitsbelastung im Alltagsgeschäft wurden als wesentliche Gründe hierfür genannt. Nur die wenigsten Unternehmen haben eigene MitarbeiterInnen, die sich mit Fördermöglichkeiten beschäftigen. Für diese Unternehmen - so geht aus den Analysen hervor - spielen Intermediäre (Technologie- und Innovationszentren, Technologieimpulsegesellschaften, regionale Innovationsmanager/-agenturen u. ä.) in ihrer Funktion als Multiplikatoren eine entscheidende Rolle. Eine beträchtliche Anzahl der befragten Unternehmen, die in der Regel als (Kern-)Partner in den Konsortien agieren, wurden von intermediären Institutionen auf die Möglichkeit der Förderung durch protec 2002+ aufmerksam gemacht und somit mobilisiert. Folgendes Zitat eines befragten Technologiezentrumsmanager verdeutlicht dies:

26 Die wichtigsten Kriterien der neuen KMU-Definition: Anzahl der Mitarbeiterlnnen < 250; Umsatz $\leq € 50$ Millionen oder Bilanzsumme $\leq € 43$ Millionen. Es werden nur eigenständige Unternehmen an diesen Kriterien gemessen, d.h. verbundene Unternehmen müssen insgesamt der KMU-Definition entsprechen, um als KMU zu gelten. 
"Ich als Technologiezentrumsmanager bin natürlich in einem Informations- und Kontaktnetzwerk drinnen, in dem auch die Förderprogramm-Thematik eine Rolle spielt. Wir haben immer wieder Mails bekommen, die auf Fördermöglichkeiten aufmerksam machten und dann ist auf einmal mit protec diese neue Förderschiene gekommen. Ich hab gesehen, da ist etwas dabei, wo wir als Impulszentrum etwas beitragen können. (...) Die Partner habe demzufolge ich informiert. Ich weiß, dass zwar sehr viele Firmen auch auf diesen Verteilern stehen, aber die Firmen - zumindest die kleineren - haben selber keine Kapazitäten bzw. Personen, die sich mit solchen Dingen beschäftigen. Hier können wir als Impulszentren etwas bewirken. Die kriegen zwar die Infomails, aber an denen geht dies vorbei, die nutzen diese Informationsquellen zu wenig."

Grundsätzlich lässt sich auf Grund von drei Ausschreibungsrunden kein eindeutiger Trend in Bezug auf die Entwicklungen in den beiden Förderlinien protec-INNO und protec-TRANS feststellen. Dennoch ist auf die stetig abnehmende Zahl von Einreichungen in der Programmlinie protec-INNO hinzuweisen, die mit 15 eingereichten Projektanträgen in der dritten Ausschreibungsrunde das bisher schwächste Echo erzielt hat. Auch wird in den durchgeführten Analysen der im dritten Monitoringbericht artikulierte Eindruck der Jury bestätigt, wonach die Projekte in der Programmlinien protec-INNO einen nur vergleichsweise geringen Innovationsgehalt aufweisen (siehe auch Kapitel 4.2.1). Insgesamt deuten die Analysen darauf hin, dass sich der Zusatznutzen der Entwicklung und Erprobung immer neuer InnovationsmanagementTools in der von protec-INNO adressierten Form langsam zu erschöpfen scheint. Die Herausforderung liegt laut ExpertInnen in der Zukunft eher darin, neue Ansätze für die Verbreitung und Diffusion von bereits existierenden Innovationsmanagement-Tools zu entwickeln, als weiterhin die Entwicklung und Erprobung neuer Tools zu forcieren. Hingegen scheint der Bedarf nach einer überregionalen Netzwerkförderung um ein konkretes Innovationsvorhaben, wie es protecNETplus darstellt, sowohl entsprechend der Analyse der Mobilisierungswirkungen von protec 2002+ als auch den ExpertInnen zu Folgedeutlich stärker gegeben.

\section{Bekanntheit von protec $2002+$}

Trotz der vergleichsweise positiven Mobilisierungswirkungen von protec 2002+ (das ausgelöste Projektvolumen ist in der Größenordnung vergleichbar mit jenem von REGplus) weist das Programm laut Expertlnnenmeinung eine relativ geringe Bekanntheit insbesondere innerhalb der Zielgruppe der KMU auf. Dies wird auch durch die Kurzbefragung der KMU FORSCHUNG AUSTRIA unter den österreichischen Gewerbe- und Handwerksbetrieben bestätigt. Von den knapp 4.000 befragten Unternehmen aus den Sektoren Bau, baunahe Gewerbe, Holz, Metall und Elektro, Chemie und Kunststoff, Nahrungs- und Genussmittel, Textil und Bekleidung sowie persönliche Dienstleistungen gaben nur knapp $3 \%$ an, das Technologietransferprogramm protec 2002+ bzW. dessen Programmlinien protec-INNO, protec-NETplus oder protec-TRANS zu kennen. Bei Betrieben mit weniger als 10 Mitarbeiterlnnen sind dies sogar nur 2,4\%, bei Betrieben mit mehr als 20 Beschäftigten ist protec 2002+ immerhin 4,2\% der UnternehmerInnen bekannt. Ähnliche Unterschiede zeigen sich auch bei der Betrachtung nach Sektoren. Während protec 2002+ bei den persönlichen Dienstleistungen (Friseure, Textilreiniger, Wäscher und Färber) mit 1,4\% aufgrund der geringen Notwendigkeit von FuE erwartungsgemäß die geringste Bekanntheit aufweist, kennen immerhin 4,8\% der Betriebe im Sektor Chemie und Kunststoff (Kunststoffverarbeiter) das Programm.

Einer der Gründe für die vergleichsweise geringe Bekanntheit von protec 2002+ unter den österreichischen Gewerbe- und Handwerksbetrieben dürfte wohl darin liegen, dass insgesamt immerhin knapp $77 \%$ der befragten Unternehmen angeben, keinen Bedarf nach Unterstützung bei ihren Innovationsaktivitäten zu haben. Dabei nimmt der Unterstützungsbedarf mit zunehmender Unternehmensgröße zu und liegt bei den Betrieben mit mehr als 20 Beschäftigten bei immerhin über einem Fünftel. Deutliche Unterschiede zeigen sich dabei auch nach Sektoren. Während beispielsweise knapp ein Viertel der Betriebe im Sektor Chemie Kunststoff angibt, Bedarf nach Unterstützung bei seinen Innovationsaktivitäten zu haben, liegt dieser Anteil im Sektor Textil und Bekleidung bei nur $8 \%$ (siehe Abbildung 3). 
Abbildung 3: Bedarf nach Unterstützung bei Innovationsaktivitäten, Anteil der Unternehmen in Prozent

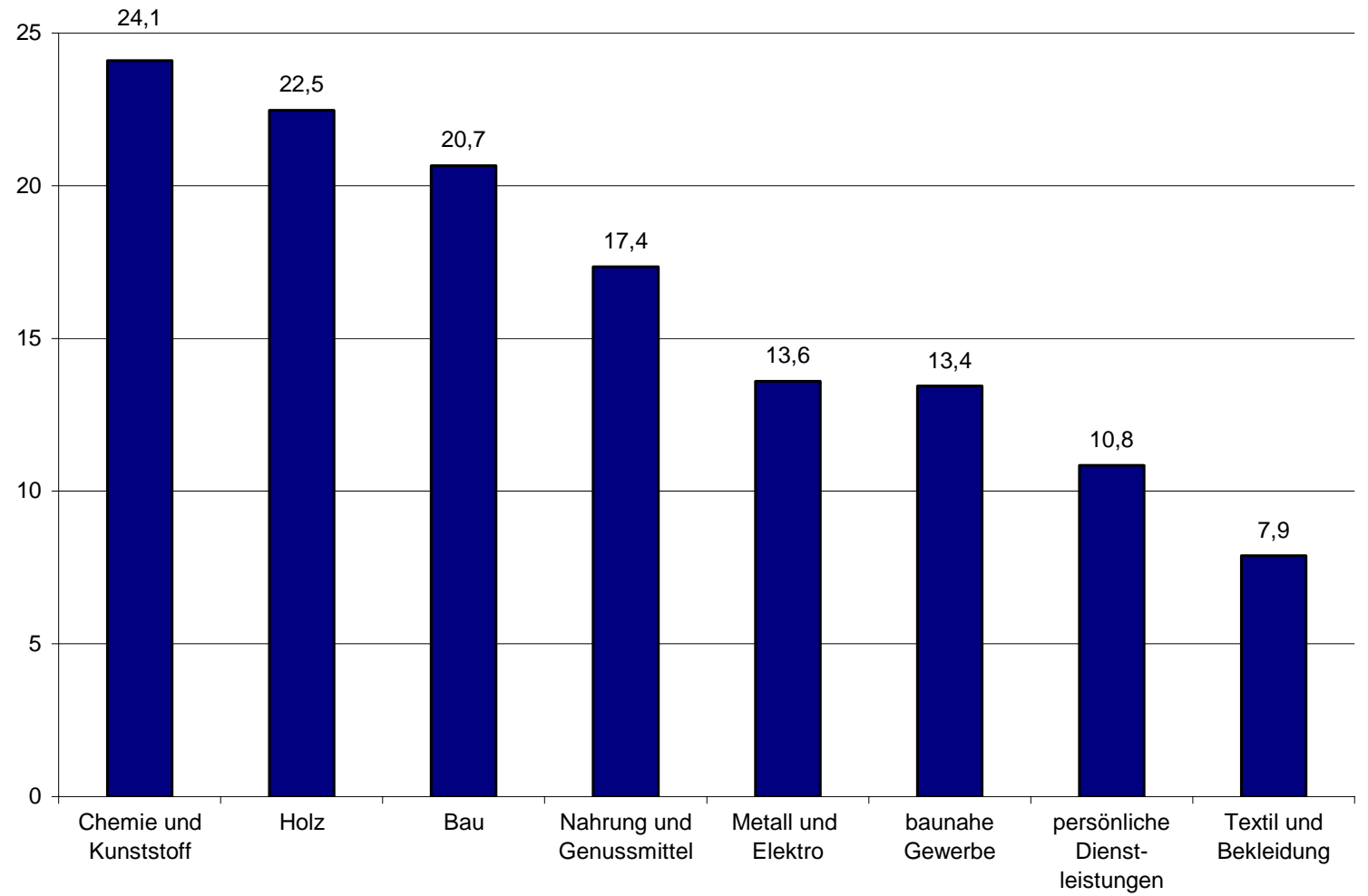

Wird die Bekanntheit von protec 2002+ nur unter jenen Unternehmen betrachtet, die einen Unterstützungsbedarf bei ihren Innovationsaktivitäten aufweisen, so ergibt sich zwar ein geringfügig optimistischeres Bild, dennoch scheint allein unter den österreichischen Gewerbe- und Handwerksbetrieben noch erhebliches Mobilisierungspotenzial für protec 2002+ zu bestehen. Von den $77 \%$ der befragten Unternehmen, die einen Bedarf an Unterstützung bei ihren Innovationsaktivitäten angeben, kennen nur knapp $4 \%$ das Technologietransferprogramm.

Die vergleichsweise geringe Bekanntheit von protec 2002+ im breiten Feld wird von ExpertInnen zum einen auf die relativ geringe Größe des Programms zurückgeführt, zum anderen auch auf die Tatsache, dass es sich bei protec 2002+ auf Grund der ausgeprägten Netzwerkund Kooperationskomponente (siehe Kapitel 3) um kein Breitenprogramm handelt. Umso bedeutender scheint die Einbindung von Multiplikatoren bzw. entsprechenden Intermediären in der „Vermarktung“ von protec 2002+ (insbesondere auch vor Ort in den Bundesländern), um zielgerichtet über das Programm informieren zu können. Mit der Wirtschaftskammer Österreich konnte diesbezüglich bereits ein wichtiger Partner gewonnen werden. Dennoch kommt insbesondere bei einem Technologietransferprogramm wie protec 2002+, das keine klar definierte und damit adressierbare Community vorfindet, sondern je nach Timing der Ausschreibung bzw. Verfügbarkeit alternativer Förderangebote unterschiedliche „Szenen“ anspricht, ${ }^{27}$ der Einbindung und Schaffung einer „strategischen Intelligenz" - insbesondere Vor-Ort in den Bundesländern - die über einen direkten Zugang zu KMU verfügt, eine besondere Bedeutung zu.

27 Siehe auch protec $2002+$, Monitoringbericht 3 (2005, S. 9) 
Wichtig scheint laut ExpertInnenmeinung dabei, dass die entsprechenden Akteure nicht nur als erste Anlaufstelle für potenzielle Antragsteller fungieren, sondern darüber hinaus auch eine sog. Erstberatung anbieten können. Studien haben gezeigt, dass eine schnelle und kontextangemessene Beratung vor Ort erheblich zur Mobilisierung der österreichischen Forschungsakteure beitragen kann. Insbesondere für unerfahrene Projektantragsteller bzw. „Neukunden“ im Bereich der Innovations- und Forschungsförderung kommt der regionalen Beratung und Betreuung eine besondere Bedeutung $\mathrm{zu}^{28}$. Darüber hinaus könnte eine verstärkte Einbindung regionaler Fördereinrichtungen in die Informations- und Beratungsaktivitäten für protec 2002+ auch einen wichtigen Beitrag zu der mehrfach geforderten sorgfältigeren Abstimmung der unterschiedlichen Förderangebote auf Bundes- und Landesebene leisten.

In diesem Zusammenhang wird von den befragten ExpertInnen sowie von den Fördernehmern auch der Mangel eines einheitlichen "Auftritts“ von protec 2002+ angesprochen. Zwar finden sich Informationen zu dem Programm auf zahlreichen Homepages (BMWA, FFG, ERP-Fonds), jedoch gibt es hier keinen Wiedererkennungswert bzw. weist protec 2002+ keine eigene Marken-Identität auf. Dies mag nicht zuletzt auch darin begründet sein, dass es keine eindeutig definierte Anlaufstelle für Informationen zu dem Technologietransferprogramm gibt. Auch eine bessere Informationsbereitstellung zu den geförderten Projekten, eine schneller erfolgende Veröffentlichung der Projektinhalte, die Bereitstellung von Informationen über die beteiligten Institutionen sowie eine stärkere Außenkommunikation mit PR-Leistungen für die einzelnen Projekte werden von den ExpertInnen angeregt.

\subsubsection{Struktur der Fördernehmer und Konsortien}

Eine Analyse der Struktur der Programmteilnehmer in Bezug auf die unterschiedlichen Partnertypen zeigt, dass protec 2002+ ein breites Spektrum von Akteuren anspricht. Dabei nehmen in der Programmlinie protec-INNO vor allem Unternehmensberater eine besondere Rolle ein (siehe Abbildung 4), die hier explizit als Zielgruppe angesprochen sind und häufig auch als Projektkoordinatoren und damit als treibende Kraft hinter der Antragstellung auftreten. Darüber hinaus sind KMU eine vergleichsweise dominante Gruppe von Teilnehmern bei protec-INNO, wobei sich deren Anteil von der zweiten zur dritten Ausschreibungsrunde sogar deutlich erhöht hat $^{29}$ - KMU beteiligen sich an protec-INNO jedoch kaum als Koordinatoren. Demgegenüber kam den Impuls- und Technologiezentren zumindest in der zweiten Ausschreibungsrunde eine wichtige Rolle nicht nur als Projektpartner, sondern vor allem auch als treibende Kraft hinter der Projektantragstellung zu, immerhin 4 der 8 geförderten Projekte wurden von dieser Gruppe angeführt. In der dritten Ausschreibungsrunde haben die Impuls- und Technologiezentren jedoch deutlich an Bedeutung verloren.

28 siehe z. B. Sheikh et al. (2004)

29 Für die erste Ausschreibungsrunde liegen keine derartigen Daten vor. 
Abbildung $4 \quad$ Verteilung der teilnehmenden Partnertypen in der zweiten und dritten Ausschreibungsrunde, protec-INNO, Anteile in Prozent ${ }^{30}$

\section{zweite Ausschreibungsrunde}

105 teilnehmende Partner

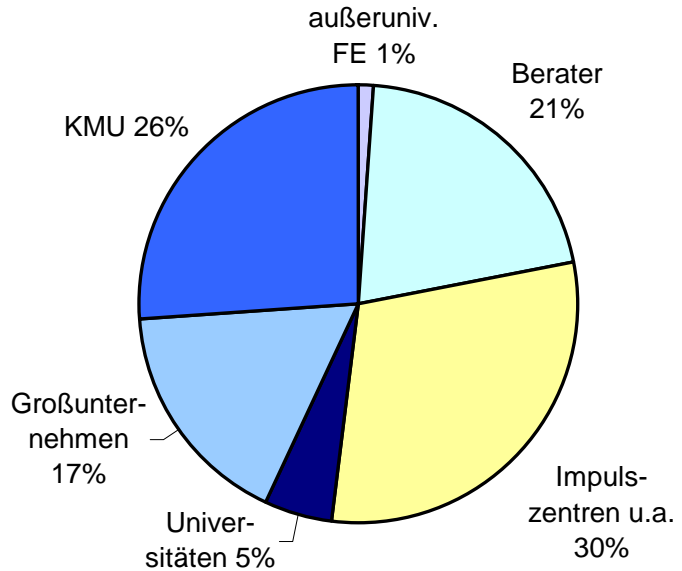

Quelle: protec 2002+, Monitoringbericht 2 (2004) dritte Ausschreibungsrunde

117 teilnehmende Partner

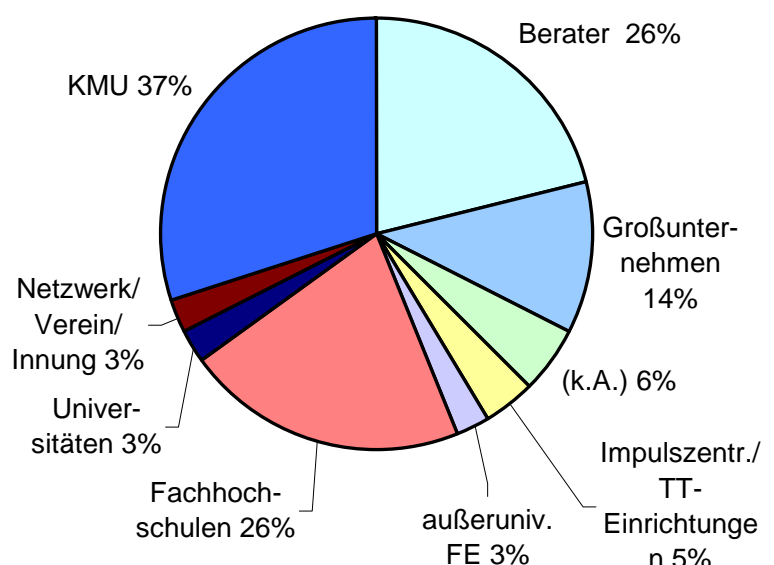

Quelle: protec 2002+, Monitoringbericht 3 (2005)

Abbildung 5 Verteilung der teilnehmenden Partnertypen in der zweiten und dritten Ausschreibungsrunde, protec NETplus, Anteile in Prozent ${ }^{31}$

\section{zweite Ausschreibungsrunde}

130 teilnehmende Partner

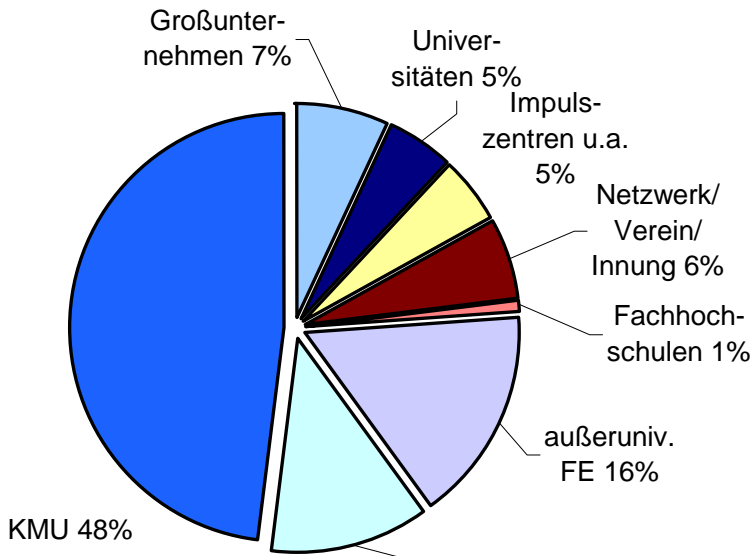

Berater 12\% dritte Ausschreibungsrunde

192 teilnehmende Partner

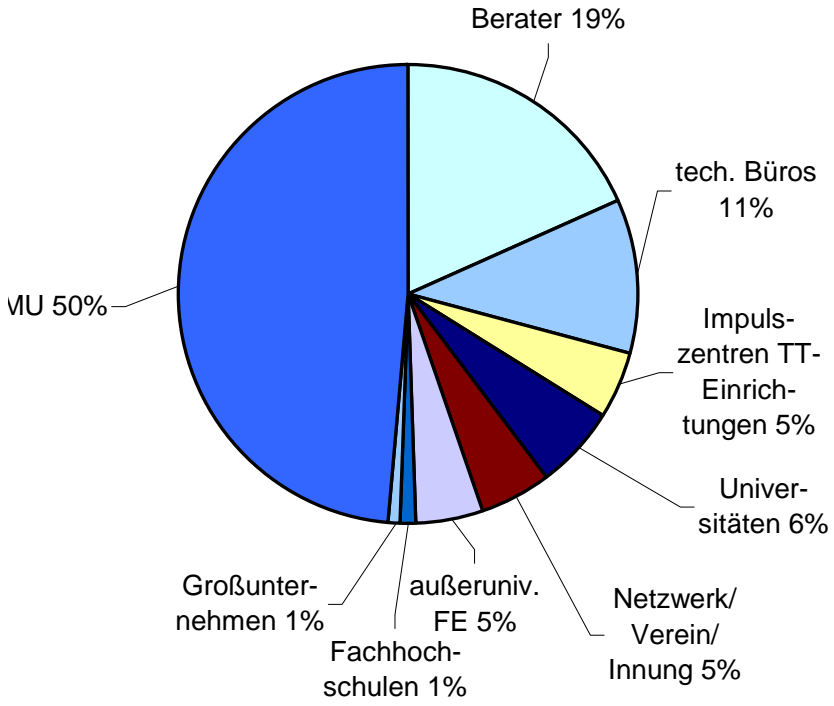

Quelle: protec 2002+, Monitoringbericht 2 (2004)

Quelle: protec 2002+, Monitoringbericht 3 (2005)

30 Entsprechende Daten für die erste Ausschreibungsrunde liegen nicht vor.

${ }^{31}$ Entsprechende Daten für die erste Ausschreibungsrunde liegen nicht vor. 
Bei protec-NETplus dominieren eindeutig kleine und mittlere Unternehmen (KMU) als Programmteilnehmer, sowohl in der zweiten als auch in der dritten Ausschreibungsrunde (siehe Abbildung 5). Dabei stellen die KMU oft auch die treibende Kraft hinter der Antragstellung bei protec-NETplus dar und sind in immerhin 3 (von 6) der geförderten Projekte der zweiten Ausschreibungsrunde und in 4 (von 15) der geförderten Projekte der dritten Ausschreibungsrunde als Konsortialführer tätig. Eher an Bedeutung verloren haben die außeruniversitären Forschungseinrichtungen; wurden in der zweiten Ausschreibungsrunde noch immerhin 2 der geförderten Projekte von solchen geleitet, gilt dies nicht mehr für die dritte Ausschreibungsrunde. Hingegen haben die Impuls- und Technologiezentren ihr Engagement an protecNETplus deutlich intensiviert. Während sie in der zweiten Ausschreibungsrunde noch primär als Projektpartner auftraten, werden in der dritten Ausschreibungsrunde immerhin 5 der insgesamt 15 geförderten protec-NETplus Projekte von Impuls- und Technologiezentren angeführt. Deutlich erhöht hat sich auch der Anteil der Berater und technischen Büros an protec-NETplus ${ }^{32}$ Insgesamt ist knapp ein Drittel (30\%) der Teilnehmer an der dritten Ausschreibungsrunde diesen beiden Gruppen zuzuordnen, wobei sogar 4 der insgesamt 15 geförderten Projekte von Beratern (3) bzw. technischen Büros (1) geleitet werden. Diese Trends sind jedoch mit Vorsicht zu beachten, da sich aufgrund der geringen Zahlen schon leichte Veränderungen stark auswirken.

Die Projekte der Programmlinie protec-TRANS weisen im Vergleich zu protec-INNO und protecNETplus einen relativ hohen Anteil an Projektpartnern aus dem universitären Bereich auf. Zudem zeigt sich eine Häufung von Akteuren aus dem Segment der FuE-Dienstleister und der Software Branche..$^{33}$

Auffällig ist in diesem Zusammenhang, dass die österreichischen kooperativen Forschungseinrichtungen kaum an protec 2002+ beteiligt sind. Die sog. ACR (Austrian Cooperative Research) Institute bilden eine Plattform für die mittelständische angewandte außeruniversitäre Forschung, Entwicklung und Innovation in Österreich und unterstützen in erster Linie Klein- und Mittelbetriebe bei ihren Forschungs- und Innovationsaktivitäten. Sie stellen somit wichtige Intermediäre für KMU im Bereich der angewandten außeruniversitären Forschung dar. Umso mehr verwundert die geringe Beteiligung dieser Institute an dem Technologietransferprogramm protec 2002+. Eine Kurzbefragung unter 11 der 18 ordentlichen Mitglieder des ACR hat ergeben, dass vier der Institute zwar Anträge für protec 2002+ gestellt haben, diese jedoch nicht zur Förderung empfohlen wurden. Weitere fünf hatten von einer Antragstellung abgesehen, weil ihnen der Aufwand für die Antragstellung bzw. für die Zusammenstellung eines entsprechenden Konsortiums zu groß erschien und je ein Institut hat auf Grund der Deminimis-Regel nicht eingereicht bzw. hatte zum gegebenen Zeitpunkt kein passendes Projekt für eine Einreichung. Eine stärkere Einbindung der ACR Institute in protec 2002+ wäre jedoch insbesondere in Hinblick auf ihre wichtige Intermediärfunktion für KMU durchaus wünschenswert und könnte eventuell durch vorzeitige Information und Sensibilisierung sowie einen entsprechenden Abbau von Hemmnissen gegenüber einer Antragstellung forciert werden.

32 in der zweiten Ausschreibungsrunde sind diese in der Gruppe der Berater zusammengefasst.

33 siehe protec 2002+, Monitoringbericht 3 (2005) 
Grundsätzlich sind die im Rahmen von protec 2002+ etablierten Netzwerke in ihrer Zusammensetzung sehr heterogen und liegen in Bezug auf die Zahl der Netzwerkpartner deutlich über der durch die Richtlinien von protec 2002+ vorgegebenen Mindestgröße von 3 (bzW. 4) ${ }^{34}$. Insgesamt liegt die Zahl der Netzwerkpartner bei den geförderten Projekten in beiden Programmlinien bei durchschnittlich 6-7 Projektpartnern (siehe Tabelle 6). Darüber hinaus weisen die Netzwerke eine sehr stark überregionale Komponente auf, die sich in allen drei Ausschreibungsrunden von protec-INNO und protec-NETplus bemerkbar macht (siehe Tabelle 7). Insgesamt handelt es sich bei 20 der 23 im Rahmen von protec-INNO geförderten und bei 17 der 26 im Rahmen von protec-NETplus geförderten Projekte um überregionale Netzwerke (d. h. die Teilnehmer kommen zumindest aus zwei unterschiedlichen Bundesländern). Damit ist es protec 2002+ gelungen, sich erfolgreich als überregionales Technologietransferprogramm zu etablieren und der Zielsetzung des Programms, nach der vor allem modellhafte Projekte mit überregionaler Signalwirkung initiiert werden sollen, gerecht zu werden.

Tabelle 7: $\quad$ Regionale Zuordnung der teilnehmenden Netzwerke

\begin{tabular}{|c|c|c|c|c|}
\hline & \multicolumn{2}{|c|}{ protec-INNO } & \multicolumn{2}{|c|}{ protec-NETplus } \\
\hline & eingereicht & gefördert & eingereicht & gefördert \\
\hline 1. Ausschreibungsrunde & \multicolumn{2}{|c|}{ 15. Sept. bis 16. Dez. 2002} & \multicolumn{2}{|c|}{ 1. Sept. bis 29. Nov. 2002} \\
\hline überregional & 18 & 8 & 22 & 3 \\
\hline ein Bundesland & 10 & 1 & 5 & 2 \\
\hline Gesamt & 28 & 9 & 27 & 5 \\
\hline 2. Ausschreibungsrunde & \multicolumn{4}{|c|}{ 3. Nov. 2003 bis 30. Jan. 2004} \\
\hline überregional & 15 & 7 & 15 & 5 \\
\hline ein Bundesland & 5 & 1 & 2 & 1 \\
\hline Gesamt & 20 & 8 & 17 & 6 \\
\hline 3. Ausschreibungsrunde & \multicolumn{4}{|c|}{ 2. Nov. 2004 bis 31. Jan. 2005} \\
\hline überregional & 13 & 5 & 18 & 9 \\
\hline ein Bundesland & 2 & 1 & 11 & 6 \\
\hline Gesamt & 15 & 6 & 29 & 15 \\
\hline Insgesamt & 63 & 23 & 73 & 26 \\
\hline überregional & 46 & 20 & 55 & 17 \\
\hline ein Bundesland & 17 & 3 & 18 & 9 \\
\hline
\end{tabular}

Zwar handelt es sich bei der überwiegenden Mehrzahl der Projekte um überregionale Netzwerke, bei denen die Partner in der Regel aus mehreren Bundesländern stammen, dennoch zeigt die Analyse der regionalen Verteilung der Teilnehmer an protec 2002+, dass die Präsenz einzelner Bundesländer sehr unterschiedlich ist. Dabei ist ein deutliches Ost-West-Gefälle auszumachen mit Wien, Oberösterreich und Steiermark an der Spitze. Aus diesen Bundesländern stammen jeweils etwa 200 (ca. $20 \%$ ) der Antragsteller an protec 2002+. Tirol, Vorarlberg und das Burgenland bilden mit weniger als $3 \%$ der Antragsteller den Abschluss (siehe Tabelle 8).

34 Förderungswerber der Programmlinie protec-NETplus können Einrichtungen des Technologietransfers in einem Konsortium mit mindestens drei Unternehmen (v. a. KMU) oder Arbeitsgemeinschaften von mindestens drei Unternehmen (v. a. KMU) sein. 
Tabelle 8: $\quad$ Regionale Verteilung der Programmteilnahme nach drei Ausschreibungsrunden, protec-INNO und protec-NETplus

\begin{tabular}{l|c|c|c|c}
\hline \multirow{2}{*}{} & \multicolumn{3}{|c}{ Anzahl der Teilnehmer } \\
\cline { 2 - 5 } Wien & \multicolumn{2}{|c|}{ eingereicht } & 68 & \multicolumn{2}{c}{ gefördert } \\
Oberösterreich & 205 & $22 \%$ & 81 & $24 \%$ \\
Steiermark & 196 & $21 \%$ & 86 & $26 \%$ \\
Niederösterreich & 193 & $21 \%$ & 32 & $9 \%$ \\
Salzburg & 121 & $13 \%$ & 26 & $8 \%$ \\
Kärnten & 56 & $6 \%$ & 8 & $2 \%$ \\
Tirol & 50 & $5 \%$ & 9 & $3 \%$ \\
Vorarlberg & 31 & $3 \%$ & 1 & $1 \%$ \\
Burgenland & 16 & $2 \%$ & 2 & $3 \%$ \\
Deutschland & 13 & $1 \%$ & 9 & $1 \%$ \\
Schweiz & 19 & $2 \%$ & 2 & $0 \%$ \\
UK & 3 & $0 \%$ & 1 & $0 \%$ \\
USA & 1 & $0 \%$ & 1 & $3 \%$ \\
k.A. & 1 & $0 \%$ & 11 & $\mathbf{1 0 0 \%}$ \\
\hline \hline Insgesamt & 29 & $3 \%$ & 934 & \\
\hline Quelle: protec 2002+, Monitoringbericht 3(2005) & & & & \\
\hline
\end{tabular}

Die starke Dominanz der Bundeshauptstadt Wien sowie der Industriebundesländer und Clusterpioniere Steiermark und Oberösterreich erscheint nicht weiter überraschend und weist darauf hin, dass es sich bei protec 2002+ nicht unbedingt um ein ganz einfaches Einstiegsprogramm handelt, sondern die im Programm gestellten Ansprüche gewisse Basiskompetenzen voraussetzen.

Insgesamt zeigt die Analyse der Programmwirkungen von protec 2002+, dass das Technologietransferprogramm vor allem den Unternehmenssektor sehr stark anspricht und dass die beteiligten Klein- und Mittelbetriebe, insbesondere bei protec-NETplus, nicht nur als Pilotanwender bzw. Projektpartner, sondern auch als Konsortialführer eine wichtige Rolle bei protec 2002+ einnehmen. Somit weist das Programm - entsprechend seinen Zielsetzungen - einen eindeutigen KMU-Fokus auf. Darüber hinaus ist die Struktur der Programmteilnehmer sehr heterogen und varriert in den bisherigen drei Ausschreibungsrunden stark, was für die Offenheit des Programms für unterschiedliche Partner- und Netzwerk-Konstellationen spricht. Auch zeigt sich, dass es protec 2002+ eindeutig gelungen ist, sich erfolgreich als überregionales Technologietransferprogramm zu etablieren; die Mehrzahl der eingereichten sowie der geförderten Projekte wurde von überregionalen Netzwerken eingebracht. Damit wird das Programm auch der Zielsetzung gerecht, dass vor allem modellhafte Projekte mit überregionaler Signalwirkung initiiert werden sollen. Eine zukünftige Herausforderung für protec 2002+ wird hingegen in der stärkeren Einbindung außeruniversitärer Forschungseinrichtungen liegen, die eine wichtige Intermediärstellung für $\mathrm{KMU}$ im Bereich der angewandten Forschung einnehmen. Darüber hinaus sind Chancen für protec 2002+ auch in der Ausschöpfung des Mobilisierungspotenzials insbesondere in den Bundesländern zu sehen, beispielsweise durch eine intensivere Einbindung regionaler Multiplikatoren und Fördereinrichtungen in die Informations- und Beratungsaktivitäten für protec $2002+$. 


\subsubsection{Bestandsaufnahme Gender Mainstreaming}

Für die Bestandsaufnahme zum Gender Mainstreaming sei vorweg zunächst einmal darauf hingewiesen, dass es sich bei protec 2002+ nicht um ein Frauenförderungsprogramm handelt. Die Verbesserung der Chancengleichheit von Frauen und Männern stellt kein explizites Ziel von protec 2002+ dar. Dennoch hat sich die Österreichische Bundesregierung dazu verpflichtet, die Strategie des Gender Mainstreaming in alle politischen Konzepte und Handlungsfelder einfließen zu lassen. Forschung und Technologie ist eines dieser Handlungsfelder. Gender Mainstreaming in Forschungs- und Technologieprogrammen bedeutet dabei, Forschungsthemen und Forschungsaufgaben systematisch geschlechtsdifferenziert zu betrachten und den Aspekt der Chancengleichheit auf allen Ebenen zu berücksichtigen. ${ }^{35}$ Insgesamt lassen sich dabei die folgenden Ebenen der Maßnahmengliederung in Hinblick auf die Implementierung von Gender Mainstreaming in bestehende Forschungs- und Technologieprogramme unterscheiden: ${ }^{36}$

Tabelle 9: $\quad$ Ebenen der Maßnahmengliederung für die Umsetzung von Gender Mainstreaming in der Forschung

Ebene der Maßnahmen

\section{Formale Ebene}

Richtlinien und Vorgaben

Inhaltliche Ebene

Integration von Gender in die Ausschreibung

Ebene der Programmumsetzung

Tägliche Praxis der Programmabwicklung

\section{Maßnahmenbeispiele}

$>$ geschlechtssensible Formulierung

$>$ geschlechtsdifferenzierte Datenerhebung

$>$ gendersensible Ausschreibung

$>$ Gender bei der Projektbeurteilung

Zusammensetzung der Jury

In Bezug auf die formale Ebene wird protec 2002+ den Anforderungen an das Thema Gender Mainstreaming durchaus gerecht. Die Leitlinien und Ausschreibungsunterlagen weisen durchgehend eine geschlechtsneutrale Schreibweise auf und mit der dritten Ausschreibungsrunde wurde auch eine geschlechtsdifferenzierte Datenerhebung auf Projektebene eingeführt. Auch wesentliche Elemente der inhaltlichen Ebene der Maßnahmengliederung in Hinblick auf die Integration von Gender in das Programm scheinen erfüllt. So wird sowohl seitens des Projektträgers (BMWA) als auch seitens des Programmmanagements auf eine geschlechterdifferenzierte Zusammensetzung der Jurys bedacht genommen; in der Regel sind bei protec-INNO und protec-NETplus zumindest ein bis zwei der insgesamt fünf Jury-Mitglieder weiblich. Die Berücksichtigung von Gender in der Projektbeurteilung rückt bei protec 2002+ deshalb in den Hintergrund, da es sich bei den Antragstellern um Institutionen und nicht um EinzelforscherInnen handelt. Auch die Durchführung spezifischer Calls mit genderrelevanten Themen erscheint bei einem grundsätzlich themenoffenen Programm wie protec 2002+ nur schwer argumentierbar. Ansatzpunkte für protec 2002+ in Hinblick auf die Umsetzung von Gender Mainstreaming scheinen hingegen auf der Ebene der Programmumsetzung gegeben. Hier wäre etwa an eine verstärkte Informationsverbreitung über entsprechende Frauennetzwerke (z.B. FEMtech, ITSalon Pour Elle etc.) bzw. eine gezielte Ansprache von Frauen in FuE Abteilungen von Unternehmen zu denken, um die Beteiligung von Frauen an protec 2002+ zu erhöhen.

\footnotetext{
35 Schrattenecker / Bodisch / Greisberger (2005)

36 Schrattenecker et al. (2004)
} 
Insgesamt sind Frauen bei protec 2002+ bisher sowohl auf Ebene der Projektleitung als auch auf Ebene der Projektmitarbeit unterrepräsentiert. Bei protec-INNO wird eines der insgesamt 6 in der dritten Ausschreibungsrunde geförderten Projekte von einer Frau geleitet und $18 \%$ der MitarbeiterInnen an den geförderten protec-INNO Projekten sind weiblich. Bei protec-NETplus werden 2 der insgesamt 13 in der dritten Ausschreibungsrunde geförderten Projekte von einer Frau geleitet, der Anteil der weiblichen Projektmitarbeiterinnen beträgt $21 \%{ }^{37}$ Allerdings ist zu betonen iegt der Frauenanteil bei protec 2002+ in der dritten Ausschreibungsrunde höher als in den meisten anderen Technologieförderprogrammen des Bundes. Insbesondere weist protec 2002+ mit durchschnittlich $16 \%$ (protec-INNO und protec-NETplus zusammengenommen) im Vergleich zu den anderen Förderprogrammen des BMWA den mit Abstand höchsten Frauenanteil auf der Leitungsebene auf (siehe Tabelle 10). Dieser liegt beispielsweise bei den FFG Basisprogrammen bzw. bei den Christian-Doppler Labors und den industriellen Kompetenzzentren mit zwischen $2 \%$ und $4 \%$ deutlich niedriger.

Tabelle 10 Frauenbeteiligung an den österreichischen Technologieförderprogrammen ${ }^{38}$

\begin{tabular}{|c|c|c|c|}
\hline $\begin{array}{l}\text { zuständiges } \\
\text { Ministerium }\end{array}$ & Förderprogramm & Führungsebene & $\begin{array}{c}\text { wissenschaftliche } \\
\text { Beschäftige }\end{array}$ \\
\hline \multirow[t]{2}{*}{ BMBWK } & $>$ node $<$ & $35,0 \%$ & $52,0 \%$ \\
\hline & GEN-AU & $11,2 \%$ & - \\
\hline \multirow[t]{3}{*}{ BMVIT } & FWF & - & - \\
\hline & Impulsprogramm AplusB & $11,1 \%$ & - \\
\hline & Kplus & $14,8 \%$ & $20,7 \%$ \\
\hline \multirow{2}{*}{ BMWA } & CD - Labor & $2,7 \%{ }^{4}$ & $21,3 \%{ }^{5}$ \\
\hline & $\mathrm{K}$ ind/net & $3,9 \%{ }^{4}$ & $13,3 \%{ }^{5}$ \\
\hline $\begin{array}{l}\text { Quelle: BMBWK, F } \\
{ }^{1} \text { Es wurden } 15 \text { vo } \\
{ }^{2} \text { bezieht sich nur } \\
{ }^{3} \text { bezieht sich auf } \\
{ }^{4} \text {. und } 2 \text {. Führun } \\
{ }^{5} \text { Junior und Senio }\end{array}$ & $\begin{array}{l}\text { Mtech, Gender Booklet } \\
\text { den } 19 \text { Programmlinien erhoben } \\
\text { If die dritte Ausschreibungsrunde } \\
\text { e im Jahr } 2001 \text { abgeschlossenen } \\
\text { sebene und Principal Scientists } \\
\text { Scientists, bzw. bei den CDG-Lab }\end{array}$ & $\begin{array}{l}\text { protec-INNO und protec-NE } \\
\text { Projekte } \\
\text { ors Post doc, DissertantInne }\end{array}$ & $\begin{array}{l}\text { mmengenommen } \\
\text { omandInnen }\end{array}$ \\
\hline
\end{tabular}

Dabei muss jedoch darauf hingewiesen werden, dass die Angaben zum Frauenanteil bei protec 2002+ ausschließlich den Antragsunterlagen der Fördernehmer entstammen und nur wenig über die tatsächlich Einbindung von Frauen in die geförderten Projekte aussagen. ${ }^{39}$ Eine systematische Herausarbeitung des Anteils der Frauen in den eingereichten Projekten nach Position und Grad der Involvierung war jedoch auch im Zuge der vorliegenden Zwischenevaluierung aufgrund des überwiegend auf Interviews basierenden Evaluierungsdesigns nicht möglich. Diesbezüglich muss auf die geplante Endevaluierung verwiesen werden. Ähnliches gilt in Hinblick auf die Analyse der geschlechtsspezifischen Allokation der Fördermittel.

protec 2002+, Monitoringbericht 3 (2005)

38 siehe Dörflinger / Sheikh (2005), Tabelle aktualisiert

39 Die Angabe in Tabelle 10 zu den anderen Förderprogrammen wurden im Rahmen entsprechender Zwischen- bzw. Ex-post Evaluierungen erhoben. 
Die Herausforderung für protec 2002+ in Hinblick auf das Thema Gender Mainstreaming wird in diesem Zusammenhang darin liegen, eine Balance zu finden zwischen dem durchaus gerechtfertigten und von ExpertInnenseite vielfach geforderten Anspruch nach einer geschlechtsspezifischen Datenerhebung differenziert nach Position, Beschäftigungsart, Einkommensgruppe, Qualifikation, Altersgruppe und Umfang der Mitarbeit der an den im Rahmen von protec 2002+ geförderten Projekte beteiligten Personen einerseits sowie dem dafür notwenigen Ressourcenaufwand andererseits. Grundsätzlich wurden in das Monitoring von protec 2002+ mit der dritten Ausschreibungsrunde erstmals genderspezifische Fragen aufgenommen, was durchaus positiv zu beurteilen ist. Dennoch sind einem Monitoringsystem wie jenem von protec 2002+, dass überwiegend auf ex ante Daten basiert und sich in erster Linie aus Angaben in den Projektbeschreibungen der Antragsteller speist, Grenzen in Bezug auf eine umfassende Analyse des Themas Gender Mainstreaming gesetzt. Eine ausführliche Analyse dieser Dimension kann nur durch eine spezifische Datenerhebung im Rahmen einer standardisierten Befragung erfolgen, die entweder Bestandteil eines umfassenden begleitenden Monitorings oder einer entsprechenden Evaluierung ist. Die entsprechenden Ressourcen sind in der Programmplanung im Vorfeld zu berücksichtigen. 


\subsection{Analyse der Projektwirkungen}

In diesem Kapitel werden die ersten Outputs und Impacts der drei Programmlinien des Programms protec 2002+ auf Projektebene analysiert. Dabei sei darauf hingewiesen, dass bei der gegenständlichen Zwischenevaluierung aufgrund der bisherigen Kürze der Laufzeit des Programms zumeist nur die kurzfristig auftretenden Wirkungen erfasst werden können. Von den 24 untersuchten Projekten wurden 3 etwa eineinhalb Jahre vor dem Zeitpunkt der Analyse abgeschlossen, 11 Projekte wurden zwischen 5 und 9 Monaten vor der Analyse beendet. Die verbleibenden 10 analysierten Projekte befanden sich zum Zeitpunkt der Zwischenevaluierung in der Hälfte bzw. gegen Ende der Projektlaufzeit. Eine Umsetzung der jeweiligen Projektergebnisse in marktfähige Produkte und Dienstleistungen bzw. eine breite Diffusionswirkung der entwickelten Methoden kann daher noch nicht erwartet werden. Ziel der Zwischenevaluierung ist es aus diesem Grund vielmehr, den bisherigen Projektverlauf zu bewerten sowie Anzeichen für längerfristige Auswirkungen hinsichtlich Verhaltensänderungen im Bereich der überbetrieblichen Zusammenarbeit und des Aufbaus neuer Kompetenzen zu untersuchen. Der Fokus der Analyse liegt dementsprechend neben der Entwicklung neuer Produkte, Dienstleistungen oder Verfahren auf Wirkungen wie Kooperationsaufbau und -kompetenz, Innovationskompetenz, Diffusion und Lerneffekten.

\subsubsection{Protec-INNO: Projektergebnisse und erste Impacts}

Insgesamt wurden 12 Projekte der Programmlinien protec-INNO untersucht, wovon 8 auf die erste Ausschreibungsrunde und 4 auf die zweite Ausschreibungsrunde entfielen..$^{40} 9$ der untersuchten Projekte waren bereits, im Sinne einer erfolgten Legung des Endberichts abgeschlossen, 2 Projekte befanden sich zum Zeitpunkt der Zwischenevaluierung in der Endphase und ein Projekt in der Hälfte der Laufzeit. Die durchschnittliche Konsortiumsgröße der 12 untersuchten protec-INNO Projekte lag bei 5 Partnern. Hinsichtlich der Zusammensetzung der Konsortien kann eine Dominanz von Technologietransfereinrichtungen und Unternehmensberatern konstatiert werden, wobei letztere Gruppe häufig die Rolle der Projektkoordination übernimmt. Die meisten Konsortien wiesen einen Mix aus neuen als auch bereits erfahrenen Kooperationen auf. Die Mehrzahl der Konsortialpartner kannte sich bereits vor dem jeweiligen protec-INNO Projekt.

Die maximale Laufzeit für Projekte des Programms protec 2002+ ist auf 24 Monate begrenzt, die durchschnittliche geplante Laufzeit der untersuchten protec-INNO Projekte lag mit etwa 20 Monaten etwas unter dieser Maximalvorgabe. Bei nahezu der Hälfte der analysierten Projekte wurde die Laufzeit jedoch während der Projektdurchführung verlängert. Die Verlängerungen waren in erster Linie auf eine Ausdehnung der Entwicklungsphase, aufgrund von Koordinationsproblemen innerhalb der Konsortien, erhöhtem Testaufwand und technischen Änderungen sowie auf Verschiebungen bei der Einbindung der Unternehmen in die Pilotphase zurückzuführen (siehe unten).

Ziel der Programmlinie protec-INNO ist die Entwicklung und Diffusion neuer Innovationsmanagementinstrumente und Beratungsansätze sowie neuer Wege ihrer Implementierung in Unternehmen (KMU). Die Programmlinie sieht dabei für alle geförderten Projekte eine Entwicklungs- und eine Implementierungsphase (Pilotphase) vor. Diese Phaseneinteilung wurde in allen untersuchten protec-INNO Projekten eingehalten. Darüber hinaus wird im Leitfaden für Förderansuchen zu protec-INNO empfohlen, bereits in der Entwicklungsphase eine Kerngruppe von Unternehmen (KMU) als aktive Partner einzubinden (Pilotanwender). Auch dieser Empfehlung sind alle der untersuchten Projekte gefolgt, zumeist in Form von kurzen Befragungen und gemeinsamen Workshops.

40 In der ersten und zweiten Ausschreibungsrunde der Programmlinie protec-INNO wurden insgesamt 17 Projekte gefördert. 
Insgesamt waren an den untersuchten Projekten durchschnittlich 5 Pilotanwender beteiligt. Die Mitwirkung der potenziellen Kunden bereits in der vorgelagerten Entwicklungsphase hat sich dabei als zielführend und wichtig herausgestellt, wie folgendes Zitat eines befragten Fördernehmers beispielhaft belegt:

„Ein größerer, zweitägiger Workshop wurde veranstaltet, bei dem alle Projektpartner teilnahmen. Firmen die miteinander im Wettbewerb standen haben extrem konstruktiv zusammengewirkt und in einem interdisziplinären Team Ideen entwickelt. Die branchenfremde Sicht hat dabei eine nicht zu unterschätzende Rolle gespielt."

Bei einigen wenigen protec-INNO Projekten kam es zu Abweichungen hinsichtlich der geplanten Einbindung von Unternehmen in der Pilotphase. In einem Fall stiegen beispielsweise mehrere Unternehmen nach Abschluss der Entwicklungsphase aus, da sie die entwickelte Methode für sich als zu wenig passend empfanden, wobei die Projektkoordination ein mögliches Ausstiegszenario bewusst von Projektstart an kommunizierte. In einem anderen Fall konnte nur die Hälfte der geplanten Anzahl an Pilotanwendern für das Projekt gewonnen werden. Diese Abweichungen sind nicht per se als negativ zu beurteilen, da sie auch zu Lerneffekten bei den betroffenen Einrichtungen führten, wie folgende Aussage eines befragten Fördernehmers belegt:
„Kleine Unternehmen im speziellen waren nur in der Entwicklungsphase involviert, haben sich die Methoden angesehen und für sich Know-how aufgebaut. Wir haben erkannt, dass diese Firmen für eine solche Beratungsmethode letztlich zu klein sind. Die größeren Unternehmen, die überblieben, befanden sich gerade in der Situation, einen Innovationsschritt machen zu wollen, haben die Methode kennen gelernt und Pilotberatungsprojekte durchgeführt."

Insgesamt funktionierte die Zusammenarbeit zwischen den Projektbeteiligten in der Regel gut. Besonders in der Entwicklungsphase fand Teamwork in Form von regelmäßigen Statusbesprechungen, Workshops und Diskussionsrunden (face to face - Austausch) statt. Darüber hinaus wurde regelmäßig telefonisch bzw. über E-Mail miteinander kommuniziert. Als wichtigste Lerneffekte und Herausforderungen der protec-INNO Projekte wurden von den befragten Fördernehmern angesichts dieses Kommunikationsaufwandes die Abstimmung des Projektablaufs und der Aufgaben mit mehreren Projektpartnern sowie die Auswahl der Pilotanwender genannt.

Inhaltlich zielten die untersuchten Projekte, wie in der Programmlinie vorgesehen, auf die Entwicklung von Innovationsmanagement-Tools und Beratungsansätzen in Konsortien ab, die darauf folgend durch einzelne Konsortialpartner mit Unternehmen als Pilotanwender getestet wurden. Zumeist wurden die Ansprüche der Benutzerfreundlichkeit, der Relevanz für KMU und des Servicecharakters als wesentliche Ziele gesetzt. Die technischen Zielsetzungen wurden bei der überwiegenden Mehrheit der Projekte erreicht, d. h. die Konzeption und Entwicklung der gemäß Förderantrag geplanten Tools, Ansätze und Mechanismen sowie deren modellhafte Implementierung konnten durchgeführt werden. Als konkrete Ergebnisse der analysierten Projekte wurden Innovationsmanagement-Tools in Form von Software (z.B. Excel-Tools), Online-Anwendungen und Beratungsmethoden bzw. -standards festgestellt. Dabei zeigt sich ein Fokus auf EDV- bzw. Onlineanwendungen. Entwickelt wurden sowohl Tools, die auf spezifische Phasen oder Stadien im Kontext des Innovationsprozesses abzielen, als auch solche, die Hilfestellungen für den Durchlauf des gesamten Innovationsprozess bieten. In der Regel wurden mehr oder minder etablierte Methoden optimiert, adaptiert oder neu kombiniert jedoch selten völlig neue Ansätze erwirkt. So wurden beispielsweise Methoden, mit welchen bereits auch international gearbeitet wird, für KMU oder auch für andere Geschäftsbereiche abgewandelt und adaptiert. Zum Teil wurden auch einzelne Ansätze mit dem Anspruch einer effizienteren Handhabung in einem Tool zusammengefasst. 
Aus diesen Beobachtungen lässt sich schließen, dass es sich bei den Ergebnissen der untersuchten protec-INNO Projekte weniger um radikale Innovationen handelt als vielmehr überwiegend um inkrementelle bzw. Anpassungsinnovationen. Dieses Analyseergebnis deckt sich mit dem im dritten Monitoringbericht kommunizierten Eindruck der Jury, wonach die eingereichten Projekte nur relativ wenig neue Ansätze gezeigt haben. ${ }^{41}$

Die entwickelten Innovationsmanagement-Tools werden vorwiegend für den „Eigengebrauch“ in einem oder mehreren an den Konsortien beteiligten Beratungsunternehmen bzw. Transferstellen eingesetzt, seltener jedoch darüber hinaus verbreitet. Als wesentliche Auswirkung der Projekte kann demzufolge die Erweiterung der Portfolios der Beratungsunternehmen festgestellt werden. Im Falle von 5 der analysierten 12 protec-INNO Projekte wurde angegeben, eine stärkere Verbreitung sei in Planung, bei 5 weiteren Projekten werden derzeit keine stärkeren Verbreitungsmaßnahmen verfolgt. Die Gründe dafür sind unterschiedlich, wie folgende Aussagen belegen:

\title{
„Ein friendly customer fehlt bislang." \\ „Der Markt ist noch nicht reif.“ \\ „Wir hatten nicht vor, das Tool zu verkaufen.“
}

Nachvollziehbare Aktivitäten zur Diffusion über das Förderprojekt hinaus lassen sich derzeit nur bei zwei der analysierten protec-INNO Projekte feststellen. Zu diesen Aktivitäten zählen beispielsweise Verbreitungsveranstaltungen und Workshops, die Einbindung von Multiplikatoren, die das entwickelte Tool anbieten und die Möglichkeit des öffentlichen Zugangs (Internet) zu den jeweiligen Tools. In diesen Fällen wurden und werden Aktivitäten verfolgt, die über den reinen Eigengebrauch der hervorgebrachten Tools und die Projektlaufzeit hinausgehen. Ein Beispiel dafür stellt folgende Aussage eines Fördernehmers dar:

\begin{abstract}
„Wir wurden bereits durch eine regionale Beratungsstelle beauftragt, zahlreiche Innovationsideen von Unternehmen in dem entwickelten Tool zu sammeln, aufzubereiten und durch Fachexperten zu bewerten. Auf Basis dieser Erfahrungen wird das Tool in weiterer Folge verstärkt in das Leistungsangebot dieser Beratungsstelle integriert."
\end{abstract}

Die Regelung der Vermarktung von Projektergebnissen ist, nach Maßgabe der protec-INNO Richtlinien, über Konsortialvereinbarungen, die u.a. Regelungen zu den Intellectual Property Rights (IPR) enthalten, geregelt. Darunter fallen beispielsweise die Nutzung und eventuelle Weitergabe der Tools, Logos und vereinzelt die gemeinsame Weiterführung von Projekt-Websites. Nur wenige Fördernehmer haben sich bis dato jedoch darüber hinaus über Verwertungsregelungen Gedanken gemacht:

„Alle Beteiligten haben grundsätzlich Verwertungsrechte, dürfen sich jedoch bei der Verwertung nicht konkurrenzieren (Konsortialvertrag). Es gibt aber keine definitive Vereinbarung, wer beispielsweise im Falle des Falles Lizenzgeber ist ... Geplant ist derzeit nichts Konkretes, man sollte wieder einmal darüber reden." 
Eine zentrale Auswirkung der protec-INNO Projekte ist der (methodische) Know-how Aufbau den die Konsortialpartner, die mehrheitlich Berater/Beratungsunternehmen darstellten, für sich erwirken konnten. Darüber hinaus wurde in vielen Projekten der Know-how Austausch zwischen Konsortialpartnern, im Sinne einer Kompetenzbündelung der zusammenarbeitenden Berater/ Transferstellen forciert und ein Konkurrenzdenken deutlich verringert. Somit konnte in vielen Fällen die Zusammenarbeit weiterentwickelt werden, wie folgende Aussagen von Fördernehmern unterschiedlicher protec-INNO Projekte illustrieren:

„Durch die bisherige Zusammenarbeit fand ein nicht zu unterschätzender Austausch zwischen den Partnern im Konsortium statt, die Transparenz wer auf welche Weise arbeitet ist größer geworden."

„Die Kompetenzen wurden durch das Zusammenspiel der Technologietransferstellen und der Unternehmensberater gebündelt, wodurch es zu einer Kompetenzbereicherung kam. Wesentliches Ergebnis ist, dass alle Beteiligten ein Stück in ihrer Arbeit weitergekommen sind. “

„Die Transferstellen haben das Bewusstsein bekommen, dass sie nicht in Konkurrenz zu den Beratern stehen und umgekehrt. Somit hat das Projekt eine Verbesserung der Zusammenarbeit bewirkt. Positiv beeinflusst hat die Zusammenarbeit auch das gemeinsame Auftreten und Agieren bei Veranstaltungen."

„Man hat mit dem Spagat zwischen Kooperation und Konkurrenz der beteiligten Transferstellen, regional und überregional, besser umzugehen gelernt."

In Einzelfällen konnten zudem bereits positive weitere Effekte der untersuchten Projekte beobachtet werden: Gründung eines neuen Beratungsunternehmens, Ausbau des Unternehmens, Erweiterung des Kundenkreises, Folgeprojekte sowie zukünftige Kooperationen mit einem oder mehreren Konsortialpartnern, die Integration eines neuen Themenfeldes in das Unternehmen und die Steigerung des Bekanntheitsgrades.

Bei den Pilotanwendern (in erster Linie KMU) konnte vor allem eine Sensibilisierung hinsichtlich des Nutzens von Optionen im Innovationsmanagement sowie der Zusammenarbeit mit Beraterfirmen und anderen Konsortialpartnern erreicht werden. Dennoch lassen sich bisher nur wenige konkrete Produktentwicklungen und Anstöße zu Innovationsprojekten bei den Pilotanwendern feststellen. Dies kann allerdings auch auf die geringe Zeitspanne zurückgeführt werden, die erst seit Projektdurchführung vergangen ist. Zudem lassen sich Diffusionseffekte nur schwer messen, je weiter sie sich von den direkten Fördernehmern entfernen.

\subsubsection{Protec-NETplus: Projektergebnisse und erste Impacts}

Insgesamt wurden 7 Projekte der Programmlinie protec-NETplus untersucht, von denen 3 Projekte auf die erste Ausschreibungsrunde und 4 auf die zweite Ausschreibungsrunde entfielen. 2 Projekte waren in der Untersuchungsperiode bereits im Sinne einer erfolgten Legung des Endberichts abgeschlossen, 2 Projekte befanden sich in der Endphase und 3 Projekte in der Hälfte der Laufzeit. Die durchschnittliche Projektlaufzeit der analysierten protec-NETplus Projekte lag bei 23 Monaten. Die untersuchten Projekte setzten sich aus Technologie- und Impulszentren, universitären und außeruniversitären Forschungsinstituten, Intermediären, Beratern, technischen Büros, KMU und Großunternehmen zusammen. Durchschnittlich waren 7,5 Partner (Konsortial- und Kooperationspartner) in die analysierten Netzwerke involviert, davon mehrheitlich KMU. In 3 der 7 analysierten Projekte traten KMU als Konsortialführer auf. ${ }^{42}$

42 In den protec-Monitoringberichten wurden Technische Büros und Beratungsunternehmen bei der Einteilung nach Organisationstypen nicht als KMU ausgewiesen. Entsprechend dieser Vorgehensweise wurde diese Einteilung auch für die gegenständliche Zwischenevaluierung übernommen. 
Die Programmlinie protec-NETplus zielt auf die Errichtung von Kooperationen und Netzwerken zur Hebung des Innovationsniveaus aller im Projekt eingebundenen Unternehmen (KMU) sowie die Durchführung innovativer Projekte in bestehenden Netzwerken. Eine klare Struktur des Netzwerkes, die Kompetenz der Partner und das Netzwerkmanagement sind dabei entscheidend. Für jedes involvierte Unternehmen (KMU) soll ein outputorientierter Innovationssprung - in Form neuer Produkte und/oder Verfahren - mit Ende des Projektes erreicht werden. Damit stellt das Programm hohe Ansprüche an die Zielgruppe, die Netzwerkkompetenz und technologische Kompetenz miteinander verbinden müssen. Es ist davon auszugehen, dass in heterogenen Netzwerken KMU und Forschungseinrichtungen vorwiegend technologische Kompetenz mitbringen und eher Defizite im Bereich der Netzwerkkompetenzen aufweisen, während zu vermuten ist, dass es bei den Intermediären umgekehrt ist. Entsprechend groß ist die Herausforderung, Projekte mit einem ähnlich hohen Anspruch im Netzwerk wie im technologischen Bereich zu konzipieren und mit einem entsprechenden Konsortium durchzuführen.

Demzufolge verwundert es nicht, dass die analysierten protec-NETplus Projekten tendenziell zwei unterschiedliche Schwerpunktsetzungen aufweisen: Einerseits existieren Projekte, bei denen der Fokus stärker auf der Entwicklung der Kooperation im Netzwerk liegt. Darunter fällt z.B. die Zusammenarbeit zwischen unterschiedlichen Akteuren zu dem Projektthema, die Entwicklung einer gemeinsamen Arbeitskultur, aber auch die Durchführung von Verbreitungsmaßnamen der Projektergebnisse. Dabei steht das Ziel im Vordergrund, durch die Zusammenarbeit im Netzwerk die Innovationsfähigkeit der Partner zu stärken. Andererseits gibt es Projekte, bei denen der primäre Fokus auf der gemeinsamen Produktentwicklung liegt und die daher einen weniger starken Netzwerkcharakter erkennen lassen. Die Analyse der sieben protec-NETplusProjekte weist dabei darauf hin, dass es insbesondere für die involvierten KMU schwierig ist, die Netzwerkkomponente mit einem gleichermaßen hohen technologischen Anspruch zu verknüpfen. Es gibt einige Projekte innerhalb welcher dies zu gelingen scheint, bei der Mehrzahl besteht jedoch eine Tendenz in Richtung Netzwerkaufbau bzw. Produktentwicklung. Die Ausrichtung der aufgebauten Konsortien ist dabei unterschiedlich. Je nach Inhalt und Ausgangslage bildeten sich Netzwerke mit Fokus auf gemeinsame Forschung und Entwicklung sowie marktnähere Netzwerke. Insgesamt erschwert diese Heterogenität zwar eine einheitliche Bewertung der protec-NETplus Projekte, sie ist allerdings insofern positiv zu beurteilen, als sie zeigt, dass protec 2002+ offen für unterschiedliche Partner- und Netzwerk-Konstellationen ist und damit die unterschiedlichen Bedürfnisse der Zielgruppe abgedeckt.

Die technischen Ziele der Projekte reichen von der Durchführung computergestützter Simulationen, der Erstellung von Planungsgrundlagen für einen spezifischen Technologiebereich und erfolgreich durchgeführten Testverfahren bis hin zur Entwicklung und Optimierung vermarktungsfähiger Produkte. Diese Zielsetzungen wurden in den untersuchten abgeschlossenen Projekten größtenteils erreicht, in den sich in der Endphase befindenden Projekten ist die Erreichung in überwiegendem Ausmaß absehbar.

Auch die Kooperationen sind erfolgreich verlaufen. Die Zusammenarbeit hat den Angaben der befragten Fördernehmer zufolge in den Projekten durchwegs gut funktioniert. Dabei hat sich gezeigt, dass vorwiegend herkömmliche Kommunikationswege (face to face, telefonisch und über E-Mail) genutzt wurden und auch am wirkungsvollsten zur Unterstützung der Zusammenarbeit waren. Der in einem Projekt geplante Einsatz einer Kommunikations- und Informationsaustauschsoftware hat sich beispielsweise nicht bewährt. Hierzu zwei Projektpartner:

Projektleiter: „Wir beabsichtigten eine Plattformsoftware zwecks Dokumentenaustauschs im Rahmen des Projekts zu verwenden. Diese ist aber de facto von der Mehrheit der Partner, insbesondere von den kleineren Unternehmen, die eine einfach und schnell zu bedienende Infrastruktur benötigen, nicht genutzt worden."

Partner: „Diese Plattform hat uns sehr interessiert, ist aber nicht genutzt worden. Im Endeffekt waren die normalen Kommunikationswege einfacher." 
Die Netzwerkarbeit gestaltet sich vielfältig. Neben der projektinternen Zusammenarbeit und Abstimmung wurden in 4 der 7 analysierten protec-NETplus Projekte - darunter abgeschlossene und laufende - zusätzlich Maßnahmen hinsichtlich eines gemeinsamen Auftritts der Konsortialpartner nach außen gesetzt. Dabei wurde auf die Etablierung des aufgebauten Netzwerkes sowie auf einen verbesserten Zugang zu Märkten abgezielt. Die Maßnahmen reichen von einem gemeinsamen Internetauftritt, der laufenden Veröffentlichung von Zwischenergebnissen über die Koordination gemeinsamer Entwicklungs- und Marketingaktivitäten bis zu Artikeln in Print- und Onlinemedien, Fachvorträgen, Messeauftritten und Veranstaltungen. Zentrale und als sehr positiv zu beurteilende Lerneffekte, die mit protec-NETplus Projekten erzielt wurden, betreffen dementsprechend auch die Netzwerkarbeit. Die Erfahrung des Arbeitens in einem Netzwerk wurde von den befragten Fördernehmern als zentral für den Erfolg des Projekts eingestuft. Insbesondere bei jenen Projekten in welchen unterschiedliche Institutionen zusammenarbeiteten, konnten Kompetenzen hinsichtlich Voraussetzungen, Steuerung und Umsetzung in Netzwerken deutlich aufgebaut und erweitert werden:

„Der Prozess der Netzwerkbildung war bereits in der Phase von der Antragstellung bis zur Genehmigung des Projekts höchst interessant. Mit der Abklärung der einzelnen Rollen und Kompetenzen hat die Netzwerkarbeit begonnen. Im Anfang war eine gewisse Ignoranz anderen Partnern gegenüber zu spüren, aber einzelne Personen haben sich regelrecht in das Projekt hineingekämpft und letztlich Akzeptanz erwirkt."

„Der Verlauf des Projekts bestätigt bisher, dass es Sinn macht, Projektpartnern Verantwortung in den einzelnen Kompetenzbereichen zuzuschreiben. Diesbezüglich kann sehr viel zurückfließen,...“

Zentrale Erfolgsfaktoren für das Gelingen der Netzwerkkooperationen bestanden dabei in der gemeinsamen Zielvereinbarung und Terminplanung, wie folgende Zitate belegen:

„Wir haben im Rahmen des Projektes gelernt, dass sich Ziele der Partner zu $100 \%$ mit den Zielen die mit dem Projekt verfolgt werden, decken müssen."

„Wenn mit Partnern im Rahmen eines Netzwerks gearbeitet werden soll, muss ein gewisser Druck aufgebaut werden und Disziplin eingefordert werden.

„Ohne eine dezidierte Terminplanung und ohne Termindruck wäre das Projekt nicht zu realisieren gewesen."

Neben den Lerneffekten im Bereich der Netzwerkarbeit gehören gerade in jenen Projekten, in denen unterschiedlichste Kooperationspartner zu einer Thematik bzw. in einem Forschungsfeld zusammenarbeiteten, der Know-how-Aufbau und -Transfer sowie der Informationsaustausch zu den am stärksten feststellbaren Auswirkungen auf Projektebene, wie die nachfolgende Aussagen der Befragten veranschaulichen.

„Die Kooperationsfähigkeiten konnten ausgebaut werden, herauszustreichen ist hier, dass die beteiligten KMU zuvor noch keine Erfahrungen in der Zusammenarbeit mit Forschungseinrichtungen hatten."

„Durch die Kooperation konnten wir Know-how dazu gewinnen, das wichtig für zukünftige Aktivitäten sein wird. “

„Aufgrund der Zusammenarbeit konnten Vorhaben angegangen werden und Erkenntnisse gewonnen werden, die alleine nicht verwirklichbar gewesen wären."

„Die Abstimmung der unterschiedlichen Zugänge und Kompetenzen der Konsortialpartner war eine neue Erfahrung. Man bekommt eine stärkere Idee davon, wie andere Einrichtungen arbeiten und welche Bedürfnisse diese bei der Zusammenarbeit haben." 
Die hohe Bedeutung der Förderung im Rahmen von protec 2002+, als Anstoß für nachhaltige und weiterführende Kooperationen, zeigt sich dabei v. a. in den geplanten und zum Teil bereits realisierten über das Projekt hinausgehenden Aktivitäten mit den Projektpartnern. In fast allen der untersuchten protec-NETplus Projekte (6 von 7) sind solche langfristigen Wirkungen der Förderung bereits jetzt feststellbar. Hierzu folgende Aussagen:

„Das Projekt hat sicherlich zu einer weiteren Nachhaltigkeit der Kooperationsbemühungen beigetragen, die Kontakte wurden gefestigt und sind auch in anderen Projektzusammenhängen sowie nach Abschluss des Projekts relevant."

„Wir haben mit denselben Partnern und einigen neuen Partnern bereits ein Folgeprojekt gestartet."

„Wir möchten mit dem selben Team, außer einem Partner, jedoch mit Unterstützung eines Forschungsinstituts, ein weiteres Projekt auf Schiene bringen und suchen derzeit nach einer Einreichmöglichkeit (...) Eine gewisse Nachhaltigkeit ist in diesem Projekt sicherlich gegeben. Wir haben gerade erst vor 14 Tagen wieder eine Sitzung gehabt, obwohl das Projekt eigentlich schon abgeschlossen ist."

„Wir haben ein Nachfolgeprojekt mit den gleichen Partnern realisiert, da der Wunsch bestand, tiefer in die Projektinhalte einzusteigen."

Die Vermarktung und die Marktaussichten für Ergebnisse aus den untersuchten protec-NETplus Projekten sind in jenen Fällen noch unklar, in denen die Weiterentwicklung bzw. Pilotierung zentraler Ergebnisse noch aussteht oder erst in Folgeprojekten durchgeführt werden soll. Insbesondere bei Projekten in denen der Netzwerkaufbau bzw. die Netzwerkarbeit im Vordergrund steht und erfolgreich forciert wird, scheint die Nutzung der Projektergebnisse bisher weniger absehbar. Ein Konsortialpartner eines sich im Abschluss befindenden Projektes nimmt wie folgt Stellung zu den Projektergebnissen:

„Die Ergebnisse sind insgesamt etwas zu forschungslastig (...) diese müssten noch überarbeitet werden, um sie etwa publizieren zu können."

„Die Suche nach der Finanzierung einer Anlage, um Ergebnisse letztendlich demonstrieren zu können, gestaltet sich immer noch als schwierig. Die Errichtung der Anlage war zwar nicht Bestandteil der Projektförderung, jedoch ein Ziel des Projektes und wäre nötig um potenziellen Investoren etwas Handfestes präsentieren zu können."

Vereinzelt konnten jedoch auch bereits neue Geschäftsfelder, Märkte und Kundengruppen erschlossen werden, wenngleich sich die Vermarktung bzw. Diffusion der Projektergebnisse, ähnlich wie bei protec-INNO auch bei protec-NETplus für die meisten Fördernehmer als schwierig gestaltet bzw. zum Teil auch noch gar nicht richtig angelaufen ist, wie folgende Zitate belegen:

„IPRs sind kein Problem, weil wir sie bei der Firma sehen, bei der die Idee entstanden ist. Das ist eindeutig. Es geht viel eher um die Finanzierung, gerade bei kleinen Firmen in diesem Bereich. Wir werden jetzt neue Wege beschreiten und wahrscheinlich zum FFF gehen wo wir aber wiederum nur als Einzelfirma hingehen können. Da wird das Netzwerk wieder auseinander dividiert. "

„Jeder überlegt für sich selbst, wo und wie das Produkt angewendet werden kann, jetzt beginnt erst die eigentliche Phase der Anwendung und Markteinführung und zwar bereits außerhalb des Projektrahmens." 
Insgesamt lässt sich schließen, dass protec-NETplus zu einer deutlich verbesserten und nachhaltigen Zusammenarbeit unterschiedlicher Akteure beiträgt, die sich mit einer gemeinsamen Innovationsthematik befassen. Neben den Lerneffekten im Bereich der Netzwerkarbeit kann ein Know-how-Aufbau und -Transfer als zentrale Auswirkung der Programmlinie erkannt werden. Die Herausforderung für die beteiligten Fördernehmer liegt dabei darin, den Netzwerk- sowie den Innovationsanspruch gleichermaßen zu erfüllen. Der Förderung im Rahmen von protec $2002+$ gibt dafür einen wichtigen Anstoß, mit deutlichen Anzeichen zu mittel- und langfristigen Wirkungen über die Projektdauer hinaus. Bereits jetzt zeigt sich, dass protec-NETplus entscheidend dazu beiträgt, Barrieren von KMU im Technologietransfer zu überwinden und ihre Möglichkeiten zum Einbezug externen technologischen Wissens auch in zukünftigen Innovationsaktivitäten merklich zu verbessern.

\subsubsection{Protec-TRANS: Projektergebnisse und erste Impacts}

Insgesamt wurden 5 der zum Untersuchungszeitpunkt geförderten 26 Projekte der Programmlinie protec-TRANS untersucht, welche bereits abgeschlossen waren. Die durchschnittliche Projektlaufzeit der analysierten Projekte betrug 6,6 Monate.

Ziel der Programmlinie protec-TRANS ist die Verbesserung der Nutzung von externen Ressourcen für KMU zur Auslösung von Produkt- und Verfahrensinnovationen sowie Dienstleistungen und zum Aufbau von Forschungs- und Entwicklungskapazität der Unternehmen. Die inhaltlichen Zielsetzungen wurden in allen untersuchten Projekten erreicht. Die konkreten Vorhaben (Produkte und Dienstleistungen) wurden plangemäß unter Einbezug externer Expertise entwickelt. Diese Expertise wurde in Form von Beratung, universitären Forschungsarbeiten, spezifischem technologischem Know-how und technischer Infrastruktur in die Projekte eingebracht. In den analysierten Projekten waren zwischen einem und drei Technologiepartner eingebunden. Die im dritten Monitoringbericht konstatierte branchenmäßige Häufung von protec-TRANS Projekten im IT-Bereich spiegelt sich auch in den untersuchten Projekten wider.

Die Mehrheit der Interviewpartner gab an, dass ein Know-how- und Technologietransfer eindeutig für die Zielerreichung des Projekts notwenig war und in sehr zufrieden stellendem Ausmaß erfolgt ist, wie folgende Aussagen zeigen:

„Für uns fand das erste Mal wirklich ein Wissenstransfer von der Uni an uns statt. Ich habe in 25 Jahren seit Bestehen des Unternehmens schon einiges zum Thema Kooperationen mitgemacht. Bei Kooperationen hat man gelegentlich den Eindruck, einer ist der Trittbrettfahrer. Aber in diesem Fall war keiner Trittbrettfahrer, sondern es hat wirklich einer vom anderen profitiert. Die beiden anderen Partner wären nicht in der Lage gewesen, das Ergebnis herzustellen."

„Ohne den Technologiepartner hätte die Aufgabe nicht gelöst werden können, da das Know-how in diesem Bereich unzureichend vorhanden war. Die Kooperation war fruchtbar. Wir würden wieder mit diesem Partner kooperieren. "

„Wir haben eindeutig von dem Know-how der Forschungseinrichtung profitiert.“

In den untersuchten Projekten wurden sowohl neue Kooperationen eingegangen als auch bestehende Kooperationen intensiviert. In 3 der 5 untersuchten Projekte wurden positive Aussagen über das Funktionieren der Zusammenarbeit mit den Technologiepartnern getätigt. In zwei Fällen gab es hingegen Konflikte in der Zusammenarbeit. Im ersten Fall wurden, laut Angabe des Technologiepartners, seitens des Förderwerbers ${ }^{43}$ während des Projektverlaufs wiederholt Forderungen technischer Art gestellt, die zuvor nicht schriftlich festgelegt waren. Der Projektpartner konnte diese Wahrnehmung nicht bestätigen. Im zweiten Fall wurde seitens des Förderwerbers angegeben, dass zwei Technologiepartner zeitweise Schwierigkeiten mit der Umsetzung ihrer im Projekt definierten Aufgaben hatten. Nichtsdestotrotz konnten die inhaltlichen Ziele in beiden Projekten erreicht werden.

\footnotetext{
${ }^{43}$ antragstellende KMU
} 
Als deutlicher Erfolg der Programmlinie kann gewertet werden, dass in zwei der untersuchten protec-TRANS Projekte Folgeprojekte mit zumindest einem Technologiepartner bereits realisiert wurden, in zwei weiteren sind sie geplant. Hier zeichnet sich eine sehr positiv zu beurteilende nachhaltige Wirkung der Förderung im Bereich des Kooperationslernens ab. Weiterhin wurde von einem Förderwerber die Initiierung eines neuen Geschäftsfelds sowie eine verstärkte Professionalisierung explizit als Impact des protec-TRANS Projekts angeführt. In einem weiteren Fall wurde eine Verbesserung der Wettbewerbssituation gegenüber Konkurrenten angegeben.

Die Vermarktung und Markteinführung der entwickelten Produkte läuft im Fall von zwei Projekten derzeit planmäßig an, im Falle eines Projekts ist diese geplant, jedoch noch nicht realisiert. In einem der untersuchten protec-TRANS Projekte kam es zu Problemen in der Verwertungsphase aufgrund von Unklarheiten über eine Nachfolgeförderung für die Weiterentwicklung des Ergebnisses, welche von dem Unternehmen vorfinanziert wurde. Diese Vorfinanzierung wurde letztlich nicht gänzlich durch die zugesagte Nachfolgeförderung abgedeckt, was die Vermarktung in Frage stellt. In einem weiteren analysierten Projekt kam es nach einem erfolgreichen Projektabschluss zu Verwertungsstreitigkeiten zwischen den Kooperationspartnern. Zur Zeit der Untersuchung wurde gerichtlich prozessiert.

Positiv anzumerken und als großer Erfolg der Programmlinie protec-TRANS zu werten ist, dass die Ergebnisse aus zwei untersuchten Projekten entsprechend patentiert werden konnten.

Grundlegendes Element der Programmlinie protec-TRANS ist der Einbezug externer Expertise zur Auslösung substantieller Produkt- bzw. Verfahrensinnovationen. Die Nutzung solch externen Know-hows hat in den meisten Fällen einen wesentlichen Beitrag zur Erreichung der Projektergebnisse geliefert, im Falle von drei Projekten lässt sich ein erheblicher Nutzen durch die Expertise der Technologiepartner für die Förderwerber feststellen. 


\section{Analyse der Programmumsetzung}

Ziel und Inhalt dieses Abschnitts sind Analysen zum Programmmanagement und zur Effizienz der Abwicklung. Zu diesem Zweck wird eingangs das Begleitsystem für die Programmsteuerung charakterisiert. Anschließend wird die Programmumsetzung hinsichtlich Projektakquisition und Ausgestaltung der Programmabwicklung dargestellt. Die Befunde fußen im Wesentlichen auf den Ergebnissen von Interviews mit den mit der Programmsteuerung bzw. -umsetzung betrauten Personen, Einschätzungen von Fördernehmern sowie ausgewählten ExpertInnen des österreichischen Innovationssystems.

\subsection{Das Programmbegleitsystem}

Ursprünglich mit experimentellem Charakter in Nachfolge des ITF-Schwerpunktes „Technologietransfer" aufgesetzt, wurde das Programm protec 2002+ angesichts der Vielschichtigkeit des Themas Technologietransfer mit einem umfassenden Begleitsystem ausgestattet, das die Qualität der Programmumsetzung sicherstellen soll. Dieses Begleitsystem (vgl. Abbildung 6) wird mit seinen zentralen Akteuren und Funktionen im Folgenden näher beschrieben.

Abbildung 6: Das Programmbegleitsystem von protec 2002+

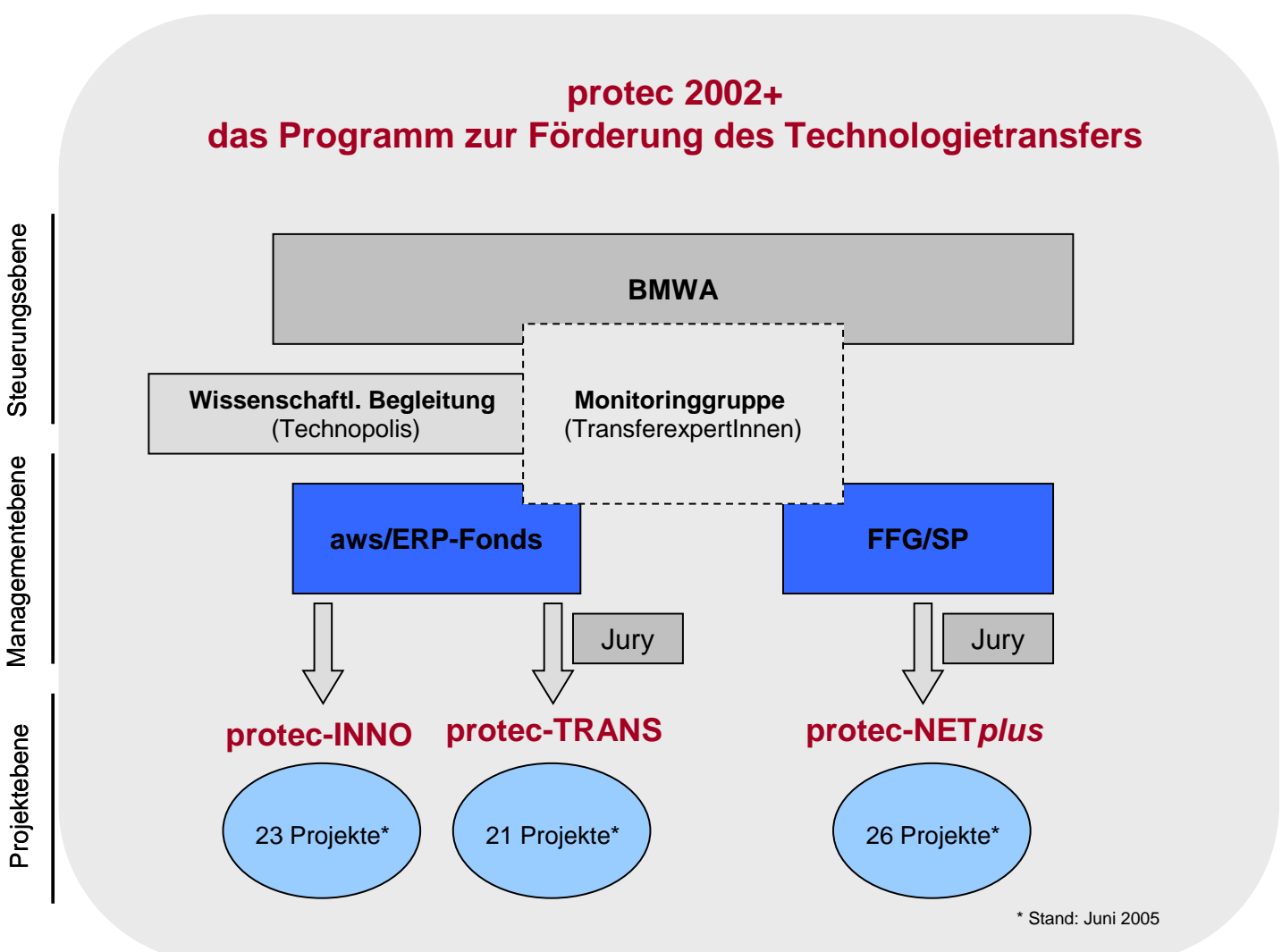


Der Programmträger BMWA, Abteilung Innovation und Transfer, der auch für die Finanzierung des Programms zuständig ist, hat mit der Programmabwicklung der Programmlinien protec-INNO und protec-TRANS den ERP-Fonds und für protec-NETplus die Forschungsförderungsgesellschaft (FFG)/ Bereich Strukturprogramme (ehemals Technologieimpulsegesellschaft, TIG) beauftragt. Diese Aufteilung gründet auf der Kompetenzverteilung zu Programmbeginn: die FFG/Bereich Strukturprogramme wurde aufgrund ihrer Kompetenzen auf dem Gebiet der Förderung kooperativer Forschung zwischen Wissenschaft und Wirtschaft ausgewählt, der ERP-Fonds aufgrund seiner langjährigen Erfahrung in der Technologieförderung für Unternehmen. Die Leistungskataloge beider Einrichtungen umfassen dabei folgende zentrale Elemente:

- den Abschluss von Förderungsverträgen mit den ausgewählten Förderungswerbern;

- die Betreuung und das Controlling der Projekte, einschließlich abschließender Projektevaluierung bis spätestens ein halbes Jahr nach Vorliegen des Endberichtes, die finanzielle Abwicklung und die Kostenkontrolle sowie die Abwicklung des Schriftverkehrs im Zusammenhang mit den genannten Aktivitäten;

- die Teilnahme an den erforderlichen Projektbesprechungen, Sitzungen der Jury und der Monitoring-Gruppe protec 2002+;

- Öffentlichkeitsarbeit in Abstimmung mit dem Auftraggeber, insbesondere Veröffentlichung der Ausschreibung in Printmedien und Erstellung von Präsentationsunterlagen.

Im Falle von protec-NETplus und protec-INNO ist auch die Vorbereitung und Durchführung der Auswahlverfahren (Überprüfung der Anträge auf formale Korrektheit, Organisation der Jurysitzungen, etc.) vorgesehen.

Jene Förderungsansuchen, welche gemäß der formalen Überprüfung teilnahmeberechtigt sind, werden von der Förderstelle einer vom BMWA eingerichteten ExpertInnen-Jury vorgelegt. Die Auswahl der ExpertInnen orientiert sich dabei an den Zielen des Programms bzw. der Programmlinien; es werden fünf VertreterInnen aus der angewandte Forschung, Industrie bei protec-NETplus, Beratung bei protec-INNO sowie von Forschungsförderungseinrichtungen als Jury-Mitglieder eingeladen. Auf Basis einer Prüfung durch die ExpertInnen-Jury wird eine Auswahl und Reihung der Förderungsansuchen getroffen. In der Folge legt die Jury dem BMWA eine Förderungsempfehlung zur Entscheidung vor. Für protec-TRANS erfolgt die technische und wirtschaftliche Begutachtung der eingereichten Projekte durch den ERP-Fonds inhouse, der seine Förderempfehlung ebenfalls an das BMWA übermittelt. Die Erkenntnisse aus den Bewertungen für die weitere Programmumsetzung werden auch der Monitoring-Gruppe zur Verfügung gestellt.

Aufgrund der Komplexität des Themas Technologietransfer und des ausdifferenzierten Förderangebots von protec 2002+ wurde auf Programmebene eine ExpertInnen-Plattform etabliert, um eine fundierte und kontinuierliche Reflexion der Programmumsetzung im Sinne eines begleitenden Monitoring der Programmlinien sicherzustellen. Das begleitende Monitoring soll eine anspruchsvolle inhaltliche Beurteilung der Programmentwicklung und damit gegebenenfalls eine rasche und flexible „Nachjustierung“ des Programms sicherstellen. Die MonitoringGruppe protec 2002+ besteht aus rund einem Dutzend Personen: jeweils zwei VertreterInnen des BMWA, der beiden für das Programmmanagement zuständigen Fördereinrichtungen und der wissenschaftlichen Begleitung; zudem sind der Verband der Technologiezentren Österreichs, das Innovationsservice Salzburg, der Wiener Wissenschafts- und Technologiefonds, die Forschungsförderungsgesellschaft/Bereich Basisprogramme sowie die Wirtschaftskammer Österreich eingebunden. Die Einbindung externer, mit der Programmumsetzung nicht befasster Experten hat sich als besonders zielführend erwiesen, da diese die erforderliche kritische Distanz zum Programm einbringen. Die Monitoring-Gruppe trifft sich einmal jährlich (2003, $2004,2005)$ jeweils im Anschluss an die Ausschreibungsrunden ${ }^{44}$. Die Mitglieder bringen ihre Expertise bzw. Perspektive in den Diskussions- und Lernprozess zur bisherigen Programm-

\footnotetext{
${ }^{44}$ protec-INNO bzw. protec-NETplus
} 
umsetzung ein. Daraus resultieren konkrete Vorschläge für die Programmabwicklung (z.B. die Adaptierungen von Ausschreibungsunterlagen) wie auch Überlegungen zur künftigen Ausgestaltung der Programmlinien von protec 2002+.

Das Beratungsunternehmen Technopolis, das auch in die Evaluierung des Vorläuferprogramms von protec 2002+ involviert war, wurde im Sinne einer wissenschaftlichen Begleitung mit der Moderation der Monitoring-Gruppe beauftragt. Darüber hinaus umfasst der Leistungskatalog von Technopolis folgende Aufgaben:

- Koordination der Datenaufbereitung für das Programmonitoring;

- Aufbereitung und Auswertung der Förderdaten in Zusammenarbeit mit den Programmabwicklungsstellen und Vorbereitung der Sitzungen der Monitoringgruppe;

- Abfassung der Monitoringberichte;

- Teilnahme an Abstimmungstreffen zwischen BMWA und Programmabwicklungsstellen;

- Unterstützung der Vorbereitung der Zwischenevaluierung vor dem Hintergrund des Programmmonitorings;

- Mitwirkung am Design des geplanten follow-up zu protec 2002+ im Lichte der Evaluierungsergebnisse;

- Vorbereitung, Moderation und Nachbereitung von themenspezifischen Workshops.

Die Ergebnisse der Programmumsetzung werden regelmäßig in Monitoring-Berichten dokumentiert, von denen mittlerweile drei vorliegen (September 2003, Oktober 2004, September 2005). Diese fassen in kompakter Form die wesentlichen Ergebnisse der Ausschreibungen in den Programmlinien zusammen und ziehen Resümee über den bisherigen Verlauf des Gesamtprogramms. Die Monitoring-Berichte stellen wichtige Programmdokumente dar, da sie nicht nur die Kommunikation des Programms protec 2002+ unterstützen, sondern auch die Ergebnisse der Programmumsetzung gegenüber den Akteuren der österreichischen Forschungs- und Technologiepolitik dokumentieren. Zusätzliche Transparenz wird erreicht, indem die Berichte auch auf der Homepage des BMWA veröffentlicht werden.

Mit der vorliegenden Zwischenevaluierung wurde eine externe Bewertung der bisherigen Programmumsetzung beauftragt, die weitere Weichenstellungen des Förderungsschwerpunktes ermöglichen soll. Eine ex-post Evaluierung nach Programmende 2006 ist ebenfalls vorgesehen.

Das Programm protec 2002+ wurde somit angesichts der Bedeutung und Vielfältigkeit von Technologietransfer und des experimentellen Ansatzes von protec 2002+ mit zahlreichen Möglichkeiten für policy learning ausgestattet. Das Programmbegleitsystem hat in seiner institutionellen Ausgestaltung durchaus Best-Practice Charakter mit Programmmanagement zur Förderabwicklung, einer wissenschaftlichen Begleitung und Monitoring-Gruppe für die Programmbegleitung, ExpertInnen-Jurys zur transparenten Projektselektion und einer externen Zwischenevaluierung. Dafür wurden bis Ende 2005 rund 6,6 \% des bis dahin ausgeschütteten Fördervolumens aufgewandt. Dies hat wesentlich dazu beitragen, dass das Programm qualitätsgesichert umgesetzt werden kann. 


\section{2. $\quad$ Ausgestaltung der Förderabwicklung}

Die Analyse der Förderabwicklung umfasst die Tätigkeiten des Programmmanagements sowie die Rahmenbedingungen für die Programmdurchführung (Projektakquisition, Antragstellung, Projektbewertung, Förderkriterien und Monitoring).

\subsubsection{Programmmanagement}

Die Programmabwicklung in den drei Programmlinien erfolgt wie bereits beschrieben durch zwei Förderstellen: der ERP-Fonds wurde aufgrund seiner langjährigen Erfahrung in der Technologieförderung für Unternehmen mit der Umsetzung von protec-INNO bzw. protec-TRANS betraut, die FFG/Bereich Strukturprogramme aufgrund ihrer Kompetenzen auf dem Gebiet der Förderung kooperativer Forschung zwischen Wissenschaft und Wirtschaft mit der Abwicklung von protec-NETplus ausgewählt.

Ein zentrales Kriterium für die Bewertung der Förderabwicklung ist die Kundenzufriedenheit, also die Perspektive der Fördernehmer. Aus Sicht der Fördernehmer arbeiten die mit der Förderabwicklung betrauten Institutionen ERP-Fonds und FFG/Bereich Strukturprogramme sehr professionell und kundenorientiert. Sowohl in der Antrags- als auch in der Abwicklungsphase wird generell die hohe Kundenorientierung der Programmabwicklungsstellen hervorgehoben. Mit den bestehenden Betreuungsstrukturen für Beratung und Informationsauskünfte individuelle Beratungsgespräche, Proposers Day oder auch Vernetzungstreffen - sind die Fördernehmer generell sehr zufrieden. Die Kontakte mit den MitarbeiterInnen der Förderstellen gestalten sich "nicht so bürokratisch wie viele andere“. Auch die Vor-Ort Besuche werden als gute Möglichkeit des „Wissenstransfers“ zwischen Projektpartnern und Fördereinrichtung geschätzt. So wird jedes protec-NETplus Projekt nach halber Laufzeit durch das Programmmanagement (Betreuer und Controller) Vor-Ort besucht; bei protec-INNO und protec-TRANS erfolgen Vor-Ort-Besuche durch MitarbeiterInnen der Förderstelle anlassbezogen.

Ungeachtet der hohen Zufriedenheit der Fördernehmer mit den derzeitigen Betreuungsstrukturen wird von den befragten ExpertInnen die Arbeitsteilung der Programmumsetzung in zwei Förderabwicklungsstellen kritisch hinterfragt. Ein zentrales Programmmanagement könnte aufgrund der zu erwartenden Größenvorteile zu Effizienzgewinnen führen (reduzierter Kommunikations- und Koordinationsaufwand, Vereinfachung des Monitorings etc.) sowie durch einen einheitlichen Auftritt nach außen (siehe Kapitel 4.1.1) die Sichtbarkeit des Themas Technologietransfer wie auch die des Programms selbst stärken. Auch die für die Zielgruppe KMU charakteristische Informationsüberforderung würde für eine Konzentration der Ansprechpartner für Technologietransfer auf Bundesebene sprechen. Was vorerst für eine Konzentration der Programmabwicklung spricht, darf jedoch allfällige „Spezialisierungsvorteile“ aufgrund der jeweiligen Kompetenzen der unterschiedlichen Abwicklungsstellen nicht verdecken.

\subsubsection{Projektakquisition}

Die Projektausschreibung in den Programmlinien von protec 2002+ erfolgt in zwei unterschiedlichen Formen. Protec-NETplus und protec-INNO werden in Form von zeitlich befristeten Aufrufen zur Einreichung (Calls) umgesetzt, wobei pro Programmjahr maximal eine Ausschreibung vorgesehen ist. Die Programmlinie protec-TRANS wird seit 2003 als offener Aufruf geführt. Die kontinuierliche Möglichkeit der Einreichung bei protec-TRANS wird sowohl von ExpertInnen als auch von Fördernehmern als angemessen bezeichnet. Die befristeten Calls werden differenziert beurteilt und daher im Folgenden genauer dargestellt. 
Die Programmlinien protec-NETplus bzw. protec-INNO werden einmal jährlich ausgeschrieben. Bisher wurden drei Ausschreibungsrunden durchgeführt. Die Ausschreibungszeiträume werden seit der zweiten Ausschreibungsrunde zwischen den Programmmanagements terminlich abgestimmt. Die Ausschreibungen sind jeweils drei Monate - von Anfang November bis Ende Januar - geöffnet. Von Seiten der Fördernehmer wird hier kritisch angemerkt, dass der Zeitraum aufgrund der zahlreichen Feiertage rund um den Jahreswechsel de facto erheblich kürzer ist.

Die Ausschreibung in Form von zeitlich befristeten Calls ist aus Sicht der Fördernehmer durchwegs für die Projektförderung im Technologietransfer geeignet. Dabei ist zu berücksichtigen, dass es sich bei den Befragten ausschließlich um erfolgreiche Fördernehmer handelt. Zwei Punkte werden mehrfach explizit als Vorteile der zeitlichen Befristung angeführt: zum einen beschleunigen die Einreichfristen den Entscheidungsprozess bei den potenziellen Partnern, insbesondere bei KMU; zum anderen stärken die Wettbewerbsbedingungen das Image des Projektes und der beteiligten Partner. Ein Projektpartner zum Thema Einreichfrist:
„Wenn man nicht irgendwo wirklich einen Druck hat - und solche Projekte entstehen einfach innerhalb von sag ich drei Wochen intensivster Arbeit - dann geschieht überhaupt nichts. Sie müssen die Partner bei der Stange halten. Gerade die produzierenden Betriebe, was die um die Ohren haben. Und wir haben gesagt: Freunde, jetzt müssen wir zusammensitzen, jetzt müssen wir das machen, weil das muss einfach am Tage $x$ fertig sein."

Generell ist davon auszugehen, dass im Rahmen befristeter Calls durchgeführten Ausschreibungsverfahren, aufgrund der wiederholten „Bewerbung“ des Programms sowie der entsprechenden Mobilisierungsaktivitäten und Öffentlichkeitsarbeit zudem die Sichtbarkeit des Programms stärken. In diesem Zusammenhang wird von einigen ExpertInnen auch eine höhere Frequenz der Calls auf zwei bis drei Ausschreibungen pro Jahr gefordert.

\subsubsection{Antragstellung}

Die Antragstellung stellt für die meisten Förderwerber einen zumutbaren Aufwand dar. So beurteilen sie das Prozedere als "im Vergleich mit anderen Ausschreibungen recht unkompliziert". „Ich habe dies als sehr benutzerfreundlich empfunden." bzw. „Ich bin solche Anträge vom FFF gewohnt [...] also ist das für mich nicht schwierig.", waren diesbezüglich typische Aussagen.

Hervorzuheben sind auch die von den befragten Fördernehmern angeführten Lerneffekte bei der Antragserstellung, die sich in der strukturierten Weiterentwicklung einer Projektidee, der Erstellung von Projekt- und Business-Plänen, aber auch allgemein im Auseinandersetzen mit den eigenen Bedürfnissen und Fähigkeiten manifestieren. Dazu beispielhaft die Aussage eines Projektpartners:

„Der Antrag hat mir einiges abverlangt. Man hat sich positionieren müssen. Man hat Aufwendungen schätzen müssen, Projekte und Kunden bringen müssen, musste nachweisen, dass es auch Kunden gibt, die dafür Geld hergeben. Somit ist eine erste Validierung gelaufen. Man hat somit einen Leitfaden, der immer durchgeht." 
Für Fördernehmer mit Erfahrung im nationalen bzw. europäischen Fördersystem ist es möglich, ein Projekt auch ohne Beratung einzureichen. Darüber hinaus haben vielfach Intermediäre wie Regionalmanager, Technologiezentren oder Transfereinrichtungen die Projektantragstellung übernommen. Ihre Erfahrungen im Fördersystem haben oft wesentlich zum Erfolg beigetragen:

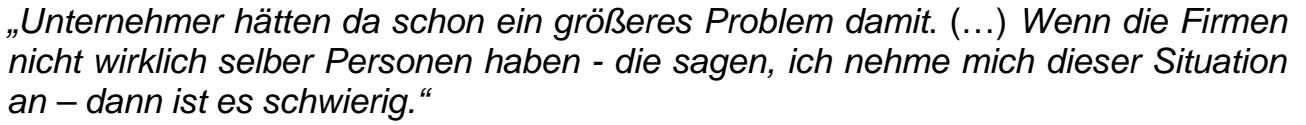

Mit den bestehenden Richtlinien bzw. Förderkriterien können die Projektziele durchaus stimmig verfolgt werden. Richtlinien und Förderkriterien werden von den Fördernehmern als durchwegs „transparent", „einfach“ bzw. „verständlich“ beurteilt. Zu dieser positiven Beurteilung hat sicher auch die Bereitstellung von Leitfäden für die Antragstellung beigetragen, die auf Basis der Erfahrungen in der Programmumsetzung kontinuierlich adaptiert werden.

Die Förderquote von bis zu $50 \%$ der förderfähigen Gesamtkosten stellt für die Fördernehmer eine durchaus attraktive finanzielle Rahmenbedingung dar: sie wird generell als angemessen beurteilt. Nicht allen Antragstellern war jedoch klar, dass auch Förderung unter $50 \%$ möglich sind. Daher wurde von den befragten Fördernehmern vorgeschlagen, diesen Umstand künftig expliziter an Förderwerber zu kommunizieren.

Die maximale Projektlaufzeit bei protec 2002+ liegt bei 24 Monaten. Diese wird von den Fördernehmern bei der Planung oft nicht ausgeschöpft. In einigen bereits abgeschlossenen Projekten zeigt sich jedoch, dass die geplante Dauer de facto nicht ausreicht. Diese ist oft auf Fehleinschätzungen der Projektpartner im zeitlichen Aufwand für Entwicklung, Netzwerkaufbau, etc. begründet. Gleichzeitig äußern sich einige Fördernehmer sehr positiv darüber, dass sich die Förderstellen im Fall begründeter Abweichungen flexibel im Hinblick auf eine Verlängerung der Projektlaufzeit zeigen. Die Beschränkung der Projektlaufzeit mit 24 Monaten ist damit grundsätzlich angemessen.

Das Berichtswesen bzw. das begleitende Controlling erfolgt im Wesentlichen durch Zwischenund Endberichte zu den geförderten Projekten. Das begleitende Controlling der geförderten Projekte umfasst das finanzielle Controlling und ein Controlling inhaltlicher Aspekte. Informationsgrundlage sind vor allem die Zwischen- und Endberichte. Mit den Zwischenberichten sollen während der Laufzeit der Projekte neben Informationen über die Mittelverwendung, Informationen über Plantreue, Organisation und Management der Projekte sowie über die Erreichung projektspezifischer Ziele gewonnen werden. ${ }^{45}$ Dass das Berichtswesen strikt gehandhabt wird, wurde von den Förderwerbern durchwegs als sinnvoll eingeschätzt. Lediglich die detaillierte Darstellung der Personalkosten wird gelegentlich als zu aufwändig bezeichnet. Des Weiteren wurde die unkomplizierte Abwicklung aller Förderstellen (z.B. Flexibilität bei Ausfall eines Partners) gelobt. Der Aufwand für Abrechnungen wird als angemessen bezeichnet. In allen Programmlinien wird dennoch Bedarf nach mehr Information bzw. Beratung in Bezug auf die Abrechnungsrichtlinien (insbes. Kostenkategorien) geäußert. Die Abrechnung erfolge aus Sicht der Fördernehmer bei protec-NETplus durchwegs prompt und verlässlich. Hinsichtlich der Abrechnungsmodalitäten bei protec-INNO und protec-TRANS äußern einige Fördernehmer Interesse an einer zügigeren Abrechnung und schlagen zudem verbindliche Fristen für Auszahlungen vor. Dazu ein Fördernehmer:

„Der einzige Wunsch bezieht sich auf eine verbesserte Kommunikation der Förderhöhe und des Zahlungszeitpunktes, um weniger "in der Luft zu hängen“. Aufbauend darauf wurde (von uns) der Vorschlag gemacht, mehr Zwischenschritte in das Programm einzuziehen. Mit jedem erledigten Zwischenschritt sollte ein Paket erledigt sein und unwiderruflich ausgezahlt werden. Auch der Zahlungszeitpunkt sollte klar und verbindlich mitgeteilt werden z.B. Überweisung binnen eines Monats nach Einreichung."

45 BMWA / ERP-Fonds / TIG (2002) 


\subsubsection{Projektselektion}

Bei den drei Förderlinien von protec 2002+ bestehen zwei unterschiedliche Vorgehensweisen in Hinblick auf die Projektbewertung. Die Komplexität der Programmlinien protec-NETplus sowie protec-INNO sprechen für den Einsatz von ExpertInnen-Jurys bei der Selektion von förderwürdigen Projekten. Die im Rahmen der Evaluierung befragten ExpertInnen betonen weiters das Element der Qualitätssicherung bei der Bewertung durch eine Fachjury, die auch mit einer höheren Selektivität bei der Projektauswahl in Verbindung gebracht wird. Unter Berücksichtigung des Aufwandes für die Programmumsetzung ist die Art der Projektbewertung durch Jurys hinsichtlich Qualitätssicherung und Aufwand durchaus adäquat.

Die Zusammensetzung der Jurys mit großteils kontinuierlicher, aber auch wechselnder Beteiligung bringt nach Einschätzung einiger ExpertInnen den Vorteil, dass unterschiedliche Perspektiven und Expertisen einfließen, die die wesentlichen Bewertungsaspekte (z.B. technische und wirtschaftliche Kriterien) abdecken. Anfangs brachte diese Heterogenität jedoch Nachteile mit sich. So gab es zu Beginn durchaus unterschiedliche Einschätzungen bezüglich der Gewichtung unterschiedlicher Bewertungsdimensionen, etwa in Bezug auf das angemessene Verhältnis von Netzwerk- vs. Technologiekompetenz bei protec-NETplus. Dazu ein Experte der Monitoring-Gruppe:

"Bei der ersten Ausschreibung hatte die Jury einfach die Schwerpunkte nicht verstanden. Klar, da waren etwa 25 Anträge, dann hat die Jury eine Vorauswahl getroffen und 12 oder 13, haben sie gesagt, sind förderbar und dann haben sie sich in der Jurysitzung nicht einigen können und haben nur 4 oder 5 gefördert. [...] Zuerst waren sie sich nicht einig und dann haben sie den kleinsten gemeinsamen Nenner gefunden."

Im Zuge der Ausschreibungsrunden konnte dann unter den Jury-Mitgliedern ein gemeinsames Verständnis hinsichtlich der Ausgestaltung und Gewichtung der Bewertungskriterien (etwa bei Netzwerken) aufgebaut werden, sodass hier von einem erfolgreichen Lernprozess gesprochen werden kann.

Die Bewertung der protec-TRANS Einreichungen erfolgt im Gegensatz zu protec-INNO bzw. protec-NETplus durch die Förderabwicklungsstelle ERP-Fonds selbst. In-house Bewertungen sind bei der Selektion und Bewertung von Förderprojekten in Bezug auf die Qualitätssicherung und den Bearbeitungsaufwand stark auf das Fach- und Erfahrungswissen der Fördereinrichtung abgestellt. Da der ERP-Fonds als Förderabwicklungsstelle auf langjährige Erfahrung in der Technologieförderung für KMU verweisen kann, erscheint die in-house Bewertung bei der Programmlinie protec-TRANS ausreichend.

In beiden Bewertungssystemen wird die Transparenz der Bewertung von den Fördernehmern in allen drei Programmlinien - als unzureichend erachtet. Die Entscheidungen seien „eher intransparent; wir haben keine Hintergrundinfos bekommen“ bzw. „(die Projektbewertung) war weder nachvollziehbar noch transparent, wir haben einfach ein Ergebnis bekommen. Es hat einfach geheißen, es wird gefördert". Ein differenziertes Feedback stellt für zahlreiche Fördernehmer eine wertvolle Information hinsichtlich der Qualität ihres Projektantrages dar. Es wird daher Übermittlung eines Evaluierungsreports mit einer inhaltlich detaillierten Darstellung der Stärken und Schwächen ihres Projektantrags angeregt. 


\subsubsection{Monitoring}

Das Monitoring stellt eine zentrale Informationsgrundlage für die Begleitung der Programmumsetzung dar. So werden etwa in den jährlichen Monitoringberichten zentrale, für die Programmsteuerung relevante Informationen hinsichtlich Mobilisierung der Zielgruppen, Projektgröße und Förderintensität, Zusammensetzung der Netzwerke, regionale Verteilung der Projekte bzw. Herkunft der Partner, Bestandsaufnahme Gender Mainstreaming, etc. aufbereitet.

Das Controlling und Monitoring der geförderten Projekte erfolgt dezentral für die Programmlinien protec-INNO und protec-TRANS beim ERP-Fonds, jenes für die Programmlinie protecNETplus bei der FFG/Bereich Strukturprogramme in ihren jeweils spezifischen Monitoringsystemen. Um die Zusammenschau der Umsetzungsergebnisse über alle drei Programmlinien hinweg leisten zu können, erfolgt die Auswertung der Monitoringdaten sowie die Aufbereitung der Programmstatistiken für die drei Programmlinien zurzeit durch die wissenschaftliche Begleitung (Technopolis). Ein einheitliches Monitoringsystem, das für alle Programmlinien gleichermaßen angewendet würde, könnte die Effizienz der Auswertung deutlich erhöhen. Das Leistungsspektrum des Programmmanagements könnte dabei um die Erstellung von Programmstatistiken - als Basis für Monitoringberichte - erweitert werden.

In Hinblick auf eine mögliche Weiterentwicklung des Monitoringssystems stellen neben Informationen über bewilligte Projekte auch Informationen über die Ergebnisse sowie Ansätze für Wirkungen in abgeschlossenen Projekten relevantes Wissen für die Programmsteuerung bzw. -umsetzung dar. Entsprechende Kurzevaluierungen (sog. ex post Assessments) könnten die Erreichung der gesteckten projektspezifischen Ziele aber auch den Beitrag zur Erreichung der Programmziele untersuchen. Diese Kurzevaluierungen, wie sie fallbeispielhaft im Rahmen der vorliegenden Zwischenevaluierung durchgeführt wurden, liefern zudem wertvolle Erkenntnisse in Bezug auf „Good Practice“ bzw. „Lessons Learned“ für die Programmumsetzung.

\section{Resümee}

Abschließend lässt sich zusammenfassen, dass die Förderabwicklung durch die beiden Programmmanagements ERP-Fonds und FFG/Bereich Strukturprogramme zur großen Zufriedenheit der Fördernehmer durchgeführt wird. Dennoch wird von ExpertInnen die Konzentration auf ein zentrales Programmmanagement gefordert, so könnten Größenvorteile genutzt und die Sichtbarkeit des Programms erhöht werden. Die Ausgestaltung der Programmabwicklung trifft im Wesentlichen auf Zustimmung bei den Fördernehmern. Die Förderkriterien und Rahmenbedingungen für die Antragstellung sowie Berichtswesen sind für die Fördernehmer durchwegs angemessen. Sie stellen einen zumutbaren Aufwand dar und wirken ansatzweise sogar verhaltenssteuernd. Adaptierungsbedarf bei den Abwicklungsmodalitäten äußern die Fördernehmer in Bezug auf rechtzeitige Bekanntgabe von Abrechnungsrichtlinien bzw. verbindlicher Zahlungsfristen.

Die Projektselektion durch ExpertInnen-Jurys bei protec-INNO und protec-NETplus sowie inhouse bei protec-TRANS bilden jeweils angemessene Bewertungssysteme. Die FachexpertInnen in den Jurys haben eingangs angesichts der Vielschichtigkeit der Bewertungsdimensionen einen Lernprozess zu durchlaufen und konnten inzwischen ein gemeinsames, praxistaugliches Verständnis aufbauen Die Kommunikation detaillierter Bewertungsergebnisse würde für die Fördernehmer zusätzliches wertvolles Feedback darstellen.

Das Monitoring auf Projektebene stellt eine zentrale Informationsgrundlage für die Begleitung der Programmumsetzung sowie hinsichtlich des zu erwartenden Beitrags zur Zielerreichung auf Programmebene dar. Ein einheitliches Monitoringsystem würde dabei eine effizientere Auswertung und Zusammenstellung steuerungsrelevanter Informationen ermöglichen. 


\section{Schlussfolgerungen und Empfehlungen}

\subsection{Zentrale Befunde für protec 2002+}

Wesentliches Ergebnis der Zwischenevaluierung ist, dass es sich bei protec 2002+ um ein erfolgreiches Förderprogramm handelt, das mit dem Thema Technologietransfer eine zentrale Funktion in der österreichischen Förderlandschaft erfüllt. Dementsprechend wird eine Weiterführung des Förderprogramms über den vorgesehenen Endzeitpunkt im Jahr 2006 hinaus empfohlen. Trotz der - angesichts der erwünschten mittel- und langfristigen Wirkungen - erst vergleichsweise kurzen Laufzeit des Programms konnten jedoch in der vorliegenden Zwischenevaluierung bereits erste Wirkungen und Erfahrungen erfasst werden, die in einer möglichen Weiterführung des Programms berücksichtigt werden sollten. Im Folgenden werden die zentralen Ergebnisse der Evaluierung kurz zusammengefasst und darauf aufbauend, Empfehlungen hinsichtlich einer Weiterentwicklung abgegeben.

\section{- Mit der Förderung des Technologietransfers erfüllt protec 2002+ nach wie vor} eine zentrale Funktion für KMU

Das österreichische Innovationssystem ist durch eine Vielzahl kleiner und kleinster Unternehmen gekennzeichnet, die angesichts des zunehmenden internationalen Konkurrenzdrucks externe Technologiequellen benötigen, um langfristig auf den Märkten mit neuen Produkten und Dienstleistungen zu bestehen. Technologietransfer verbessert den Austausch zwischen den Akteuren im Innovationssystem und trägt zur notwendigen Verwendung von FuE-Ergebnissen bei, stärkt die Innovationskraft von Unternehmen und unterstützt FuE-Einrichtungen beim Verständnis der Anforderungen industrieller Innovationsaktivitäten. Gerade bei kleinen Unternehmen bestehen jedoch entscheidende Hemmnisse, externe Quellen in Anspruch zu nehmen. Die Evaluierungsergebnisse haben gezeigt, dass das Förderprogramm des BMWA protec 2002+ in entscheidendem Maße dazu beitragen konnte, solche Hemmnisse zu verringern. Bereits durch den Prozess der Antragstellung entsteht für die Zielgruppe die Notwendigkeit, sich mit ihren Bedürfnissen, Stärken und Schwächen im Innovationsbereich auseinanderzusetzen sowie mit entsprechenden Technologiegebern in Interaktion zu treten. Durch die konkrete Projekterfahrung können Kompetenzen im Bereich der Vernetzung mit anderen Unternehmen und Forschungseinrichtungen aufgebaut werden, die bereits dazu geführt haben, dass längerfristige Kooperationen entstanden sind. Zudem wird ein Verständnis für die Denkweise und Kommunikation mit Partnern aufgebaut. Dieser Prozess ist zwar während des Projekts zeitaufwendig, trägt aber gleichzeitig zur Innovationsaktivität auf eine Art und Weise bei, die aus einem einzelnen Unternehmen heraus nicht möglich wäre. Die Projektförderung von protec 2002+ entspricht dem aktuellen Verständnis über die Wirkungsweisen von Technologietransfer. Die längerfristige Zusammenarbeit unterschiedlicher Partner ermöglicht direkte, rekursive Interaktion und damit gemeinsame Lernprozesse, die zur langfristigen Kompetenzsteigerung aller Beteiligten beitragen können.

\section{- $\quad$ Eine Förderung des Technologietransfers auf Bundesebene ist notwendig}

Aufgrund der unterschiedlichen Formen, in denen Technologietransfer durchgeführt wird, kann es kein einzelnes Förderinstrument geben, das die Notwendigkeiten des Technologietransfers in seiner Breite abdecken. Protec 2002+ nimmt im Bereich Technologietransfer in Österreich eine wichtige Funktion ein, indem es (durch die Ausgestaltung als Call) eine Signalwirkung ausübt und - in Ergänzung zu Maßnahmen auf Bundesländerebene - den überregionalen Technologietransfer in besonderer Weise unterstützt. Das Monitoring der Projekte zeigt, dass die Netzwerke eine sehr stark überregionale Komponente aufweisen, die sich in allen drei Ausschreibungsrunden von protec-INNO und protec-NETplus bemerkbar macht. 37 der 49 in diesen beiden Programmlinien geförderten Projekte werden von Konsortialpartnern aus mehr als einem Bundesland durchgeführt. Damit ist es protec 2002+ gelungen, sich erfolgreich als überregionales Technologietransferprogramm zu etablieren und der Zielsetzung des Programms, nach der vor allem modellhafte Projekte mit überregionaler Signalwirkung initiiert werden sollen, gerecht zu werden. 
In der Bundesförderung, die keinen regionalen Zielen entsprechen muss, gibt es zudem die Möglichkeit, das Gewicht stärker auf die Erzielung radikalerer Innovationen zu legen und damit auch riskantere Projekte zu fördern, deren Misserfolgsquote zwar höher liegen mag, die aber bei Gelingen auch in deutlich höherem Maße zur Wettbewerbsfähigkeit österreichischer Unternehmen beitragen können. Das Ziel eines spezialisierten Programms wie protec 2002+ kann es nur sein, Projekte mit einem spezifisch höheren Risiko zu unterstützen, wenn sie gleichzeitig einen höheren potenziellen gesellschaftlichen Nutzen bieten. Die im Vergleich zu anderen Fördermöglichkeiten höhere Förderquote ist zum einen durch den höheren Anspruch hinsichtlich der Vernetzung und zum anderen durch den Experimentalcharakter der durch protec 2002+ unterstützten Projekte gerechtfertigt.

\section{- Die thematischen Schwerpunktsetzungen der Programmlinien treffen im Wesentlichen die Bedürfnisse der KMU}

Mit der Zielgruppe eher kleiner und innovationsschwacher Akteure, denen der Einstieg in verwandte und neue Technologiefelder ermöglicht werden soll, trifft das Programm den Bereich, der von den ExpertInnen als die wichtigste Aufgabe von Technologietransfer angesehen wird. Vor dem Hintergrund einer vielfach fehlenden Kooperationsbereitschaft kleiner und mittlerer Unternehmen, insbesondere mit wissenschaftlichen Einrichtungen setzt die Förderung von Netzwerken bzw. des arbeitsteiligen Innovationsprozesses genau richtig an. Die Analysen im Zuge der Zwischenevaluierung haben gezeigt, dass das Technologietransferprogramm protec 2002+ vor allem den Unternehmenssektor sehr stark anspricht. Dabei nehmen die beteiligten KMU nicht nur als Pilotanwender bzw. Projektpartner, sondern - insbesondere bei protecNETplus - auch als Konsortialführer eine wichtige Rolle in dem Programm ein. Somit weist das Programm - entsprechend seinen Zielsetzungen - einen eindeutigen KMU-Fokus auf.

Dies erscheint umso bedeutender, als protec 2002+ unter den österreichischen Förderprogrammen mit Bezug zum Technologietransfer das einzige Programm darstellt, dass sich explizit an die Zielgruppe KMU richtet. Damit unterscheidet es sich von Förderprogrammen wie REGplus, FHplus oder prokis, bei denen zwar auch Netzwerke mit heterogenen Akteursstrukturen gefördert werden, die sich jedoch primär an andere Zielgruppen (regionale Technologie-/ Innovationszentren, $\mathrm{FH}, \mathrm{ACR}$ ) richten. Obgleich alle diese Fördermaßnahmen auf einen wesentlichen KMU-Nutzen zielen, ist protec 2002+ deutlich stärker direkt auf diese Zielgruppe gerichtet und nicht im Umweg über die Intermediäre. Somit stellt protec 2002+ eine wichtige Säule im österreichischen Technologietransfersystem, insbesondere für KMU dar, die immerhin 99,6\% aller Unternehmen der marktorientierten Wirtschaft darstellen und deren Unterstützung in Hinblick auf die Stärkung der Innovationsfähigkeit von Unternehmen nicht zuletzt auch in der Strategie 2010 des Rates für Forschung und Technologieentwicklung eine hohe Bedeutung beigemessen wird.

\section{- Heterogene Partnerstrukturen sind wichtig für den Projekterfolg (offener Zugang)}

Die neueren Ergebnisse der Innovationsforschung zeigen, dass Innovationen vor allem an den Schnittstellen entstehen, dort wo unterschiedliche Sichtweisen und Herangehensweisen aufeinander treffen und sich gegenseitig befruchten. Protec 2002+ ist es in diesem Zusammenhang gelungen, sehr heterogene und zum Teil vergleichsweise große Netzwerke, zu generieren, die bereits einen hohen technischen bzw. inhaltlichen Zielerreichungsgrad erkennen lassen. Insgesamt setzten sich die im Rahmen der Programmlinien protec-INNO und protec-NETplus geförderten Projekten aus durchschnittlich 6 - 7 Partnern und zumindest 3 unterschiedlichen Partnertypen (KMU, außeruniversitäre Forschungseinrichtung, Universität, Fachhochschule, Netzwerke/Cluster, Impulszentren und Technologietransfereinrichtungen, Beratungsunternehmen etc.) zusammen. Gerade dieser Heterogenität bzw. der entsprechenden Offenheit des Programms für unterschiedliche Partner- und Netzwerk-Konstellationen kommt dabei eine zentrale Bedeutung in Hinblick auf den Programmerfolg zu. Insbesondere jene Projekte, in denen unterschiedlichste Kooperationspartner zu einer Thematik bzw. in einem Forschungsfeld zusammenarbeiteten weisen die am stärksten feststellbaren Auswirkungen im Bereich Know-how-Aufbau und -Transfer sowie Informationsaustausch auf der Projektebene auf. Dies gilt in besonderem Maße für die protec-NETplus Projekte. 


\section{- Die Netzwerk- und Kooperationskomponente ist zentral für das Programm}

Die Netzwerkkomponente wurde in protec 2002+ eingeführt, weil sich die Programmverantwortlichen aufgrund der Vielzahl von Studien zu positiven Effekten durch Kooperationen und Netzwerke in Innovationsaktivitäten Zusatzwirkungen bei den Fördernehmern versprachen. Kurzfristiges Ziel der Kooperations- und Netzwerkförderung ist die Bündelung von Forschungskapazitäten, mittelfristiges Ziel eine schnellere Umsetzung von Forschungsergebnissen in Innovationen in Unternehmen und langfristiges Ziel die Entwicklung einer dauerhaften Zusammenarbeit zwischen Wissenschaft und Wirtschaft. Die Evaluierungsergebnisse deuten darauf hin, dass die Netzwerk- und Kooperationskomponente eine zentrale Rolle für den Erfolg des Programms spielt und die Erwartungen damit erfüllt wurden. Die hohe Anzahl erfolgreicher Anträge aus den Bundesländern mit intensiver Clusterförderung weist jedoch auch darauf hin, wie schwierig es ist, Netzwerke ohne eine bereits bestehende Kooperationserfahrung aufzubauen.

Die hohe Bedeutung der Netzwerkförderung im Programmerfolg macht auch deutlich, dass es sich bei protec 2002+ nicht um ein Breitenprogramm handelt, sondern um ein Programm, dessen Funktion in der Impulssetzung liegt. Das Programm soll Anreize für spezifische, längerfristige Verhaltensänderungen der beteiligten KMU und Forschungseinrichtungen im Sinne des Engagements in Innovationsnetzwerken geben. Zudem werden über die beteiligten Konsortialpartner positive Erfahrungen ebenso wie technologische Ergebnisse an ein breiteres Publikum disseminiert. Um diese Impulse für das österreichische Innovationssystem in spürbarer Form geben zu können, sollte das Programm jedoch eine breitere Auslegung erfahren. Dies bezieht sich zum einen auf die Differenzierung in drei unterschiedliche Programmlinien, die zu einer Aufteilung der Gesamtwirkung führt, und zum anderen auf die gemeinsame Aufstellung mit anderen Programmen, die ebenfalls auf Netzwerkbildung zielen. Beide Punkte werden unten weiter ausgeführt.

\section{- Das Programmbegleitsystem und Programmmanagement weisen Best-Practice Charakter auf}

Das Programm protec 2002+ wurde angesichts der Bedeutung und Vielschichtigkeit des Themas Technologietransfer mit zahlreichen Möglichkeiten für policy learning ausgestattet, die wesentlich dazu beigetragen haben, ein Programm mit experimentellem Charakter qualitätsgesichert und erfolgreich umzusetzen. Das Programmbegleitsystem hat in seiner institutionellen Ausgestaltung durchaus Best-Practice Charakter mit Programmmanagements zur Förderabwicklung, einer Monitoring-Gruppe und wissenschaftlichen Begleitung für die Programmbegleitung, ExpertInnen-Jurys zur transparenten Projektselektion und einer externer Zwischenevaluierung. Dafür wurden bis Ende 2005 rund 6,6 \% des bis dahin ausgeschütteten Fördervolumens aufgewandt.

Die Förderkriterien und Rahmenbedingungen für die Antragstellung sind durchwegs angemessen. Der Aufwand für die Antragstellung für die erfolgreichen Fördernehmer ist zumutbar und führt ansatzweise sogar zu verhaltenssteuernden Effekten. Der Förderabwicklung durch die beiden Programmmanagement-Stellen ERP-Fonds und FFG ist eine hohe Professionalität auszustellen, die sich in der Zufriedenheit der Fördernehmer niederschlägt. Allerdings stellt sich die Frage, ob durch die Aufteilung auf zwei Förderstellen nicht Größenvorteile verschenkt werden und die Sichtbarkeit des Programms bei der Bündelung in einer Abwicklungsstelle erhöht werden könnte. 


\subsection{Herausforderungen für die Weiterentwicklung des Programms}

Die geschilderten zentralen Befunde für protec 2002+ legen eine Weiterführung des Programms über den vorgesehenen Endzeitpunkt hinaus nahe. Dennoch sind mit einer möglichen Fortsetzung von protec 2002+ Herausforderungen verbunden, die im Folgenden näher beschrieben werden und die in der weiteren Programmplanung Berücksichtigung finden sollten:

\section{- Weiterentwicklung der thematischen Schwerpunkte von protec 2002+}

Hinsichtlich der oben angesprochenen Wünschbarkeit eines breiteren Wirkungsgrads von protec 2002+ aufgrund der Bedeutung von direktem, vernetztem Technologietransfer für das österreichischen Innovationssystem und angesichts der begrenzten finanziellen Ausstattung des Programms, erschwert die Aufteilung in drei Programmschwerpunkte die Sichtbarkeit des Programms nach außen. Auf der Basis der oben dargestellten Evaluierungsergebnisse wird daher an dieser Stelle angeregt, bei einer Weiterentwicklung von protec 2002+ eine Umschichtung des Budgets zwischen den Programmlinien zu überdenken. Die Untersuchung des Programmdesigns und der Projektergebnisse des Schwerpunkts protec-INNO geben Hinweise darauf, dass die Notwendigkeit einer Förderung der Neuentwicklung von Innovationsmanagement-Tools nicht mehr in hohem Maße gegeben ist. Die Programmlinie protec-INNO hat in diesem Bereich in den vergangenen Jahren wertvolle Pionierarbeit geleistet und wichtige Anstöße gegeben. Die Schwerpunktsetzung erscheint jedoch inzwischen ihre Aufgabe erfüllt zu haben. Auch die Analyse der Mobilisierungswirkungen von protec 2002+ deutet darauf hin, dass die Entwicklung und Erprobung von Innovationsmanagement-Tools sich in der von protec-INNO adressierten Form langsam zu erschöpfen scheint.

Der Bedarf nach einer überregionalen Netzwerkförderung für ein konkretes Innovationsvorhaben, wie es protec-NETplus darstellt, scheint hingegen auch entsprechend der Analyse der Mobilisierungswirkungen von protec 2002+ eindeutig gegeben. Vom überwiegenden Teil der ExpertInnen wird der Mehrwert im Bereich protec-Netplus als nach wie vor sehr hoch bewertet, da die Kooperationsbereitschaft zwischen KMU trotz vieler Anstrengungen immer noch als gering einzuschätzen ist. Die Verbindung von Netzwerk- und Innovationsaktivitäten ist zwar anspruchsvoll, angesichts des inzwischen anerkannten Nutzens von Innovationsnetzwerken leitet sich aber gerade daraus ein nach wie vor wichtiger Förderbereich ab.

Die Förderlinie protec-TRANS kann als unkomplizierte Einstiegsmöglichkeit für KMU in den Technologietransfer charakterisiert werden. Für eine ganze Reihe der befragten Unternehmen wurde durch die Teilnahme an protec-TRANS zum ersten Mal die Zusammenarbeit mit einer Universität oder Forschungseinrichtung realisiert.

Aus diesen Gründen wird eine Weiterführung der beiden Programmlinien protec-NETplus und protec-TRANS empfohlen, allerdings sollten die Stärken des Programmschwerpunkts protecINNO übernommen werden. Dazu gehört vor allem die Diffusion und Integration von Innovationsstrategien. Weiters wird empfohlen, die Förderung der Programmschwerpunkte explizit um die folgenden Bereiche zu erweitern:

- Produktfindung (hierbei kann auf Erfahrungen des Austria Wirtschaftsservice zurückgegriffen werden);

- Umgang mit Intellectual Property Rights (Recherche, Einsatzbedingungen, Internationalisierung);

- Strategische Markterschließung (Identifizierung nationaler und internationaler Märkte, Zugangsbedingungen, Wettbewerbsstrukturen, Nachfragesituation).

Eine Förderung in diesen Bereichen könnte zum Beispiel in der Form eines Zuschusses zu Coaching- oder Mentoringmaßnahmen erfolgen. Diese würden die Projekte nicht über die gesamte Laufzeit begleiten, sondern gezielt zu Beginn und/oder zu bestimmten Meilensteinen von externen ExpertInnen für eine begrenzte Zeit dazu gekauft werden. 


\section{- Nutzung von Synergiepotenzialen zu anderen Programmen mit Netzwerklinien}

Im Vergleich zu den Mitteln, die für die indirekte FuE-Förderung und einzelbetriebliche Projektförderung eingesetzt werden, ist die finanzielle Ausstattung in der verhaltenssteuernden Innovationsförderung in Österreich eher gering ausgeprägt. Dazu gehören Förderinstrumente, die stärker auf eine bestimmte Verhaltensänderung der Fördernehmer wie z.B. die Durchführung von FuE in Kooperationen abzielen. Protec 2002+ zeichnet sich gegenüber anderen Programmen mit Netzwerkkomponente dadurch aus, dass mit dem Kooperationsansatz nach wie vor bestehende Hemmnisse der KMU in Bezug auf arbeitsteilige FuE-Aktivitäten angesprochen werden. Mit dem Einbezug des Technologietransfers zwischen Unternehmen ist protec 2002+ deutlich anwendungsorientiert. Eine besondere Stärke des Programms liegt in der Förderung des überregionalen Technologietransfers. Das Programm zielt auf eine spezifische Unterstützung der Zielgruppe KMU. Damit richtet sich das Förderprogramm auf die Unterstützung des direkten Technologietransfers in Form von innovationsorientierten Projekten, die auf interaktivem Austausch von Wissen und Technologien aufbauen.

Eine gewisse Überschneidung zeigt sich in der Programmlinie protec-Netplus mit den Netzwerklinien der Programme REGplus und FHplus. Trotz unterschiedlicher Zielrichtungen entstehen zum Teil (in einzelnen Programmlinien) ähnliche Akteurskonstellationen - KMU-Netzwerke unter Einbindung regionaler Transfereinrichtungen oder Fachhochschulen - mit einem jeweils thematisch äußerst heterogenen Projektportfolio. Insgesamt sind die finanziellen Mittel, die in Österreich in technologie- und innovationspolitischen Programmen im Bereich Kooperation und Vernetzung eingesetzt werden, im internationalen Vergleich jedoch relativ niedrig. Die Vielfalt an Programmen heißt dementsprechend nicht, dass es eine zu hohe Förderung in dem Bereich gibt, aber möglicherweise eine zu kleinteilige. Aufgrund der unterschiedlichen Ausrichtung der Programme würde eine Zusammenlegung keinen Sinn machen. Dennoch gilt es auszuloten, inwieweit Synergiepotenziale zwischen den Programmen stärker genutzt werden können, um den Zielgruppen einen leichteren Zugang zur Förderung zur ermöglichen. Dazu gehören in einem ersten Schritt gemeinsame Vermarktungsaktivitäten. Langfristig wäre anzudenken ein gemeinsames Dach für die Förderung heterogener Innovationsnetzwerke mit unterschiedlichen Zielgruppen zu schaffen, unter dem es unterschiedliche Förderlinien und Förderzugänge gibt.

\section{- Förderung der Diffusion und Markteintritt}

Positiv ist festzuhalten, dass die untersuchten Projekte aller drei Programmlinien fast durchwegs ihre Ziele technischer oder inhaltlicher Art erreicht haben. In einer Reihe von Projekten wurden allerdings im Anschluss an die Produkt/Dienstleistungsentwicklung Schwierigkeiten bei der Verwertung der Ergebnisse und beim Markteintritt festgestellt. Aufgrund der EU-Beihilferegelung ist es trotz des Fokus auf Innovation und Marktfähigkeit nicht möglich, in protec 2002+ die Markteinführung neuer Produkte und Dienstleistungen zu fördern. Diese Problematik gilt nicht nur für protec 2002+, sondern generell für Innovationsförderprogramme. Gerade kleine und Kleinstunternehmen stehen oft vor der Schwierigkeit nach gelungenem Abschluss eines Forschungsprojekts die kostenintensive Markteinführung zu bewältigen. Häufig fehlt Know-how zur Abschätzung der Marktchancen und des Marktzugang insbesondere auf internationalen Märkten. Die Vermarktungsphase in Innovationsprozessen ist aber auch der Bereich, der aus beihilferechtlichen Aspekten in der Innovationsförderung am problematischsten ist. Es sind innovative Konzepte erforderlich, die sich auf Unterstützungsangebote im Bereich der Produktentwicklung und Prozessoptimierung, der zu stärkenden Marktkompetenz der KMU und der Durchsetzung innovativer Produkte am Markt richten (z.B. bei der Prototyp-Entwicklung), ohne dass die EU-Beihilferegelungen verletzt werden. Möglichkeiten in diesem Spektrum könnten wie oben vorgeschlagen in der Förderung von Marketingberatungen (analog zu den Innovationsberatern) oder der Unterstützung über Mentoring-Maßnahmen bei der strategischen Markterschließung ihrer neuen Produkte/Dienstleistungen liegen. Dazu gehört auch die Aufnahme von Unterstützungsmaßnahmen hinsichtlich der den protec 2002+ Projekten vorgelagerten strategischen Produktfindung. 


\section{- Unterstützung der Internationalisierung der Fördernehmer}

Bisher hat protec 2002+ noch nicht in großem Maße dazu beigetragen, Internationalisierungsaktivitäten von KMU zu forcieren. Eine weitere Öffnung für internationale Partner wird von Fördernehmern und ExpertInnen zum überwiegenden Teil begrüßt. Der Schwerpunkt sollte jedoch nach wie vor auf der Förderung des nationalen Technologietransfers liegen, das heißt Internationalisierung sollte nicht zu einer notwendigen Voraussetzung für eine Förderung werden. Auch im derzeitig bestehenden Rahmen gibt es Möglichkeiten, funktionierende Anreize für die Internationalisierung der protec 2002+ Netzwerke zu setzen wie das Beispiel PRO INNO II zeigt. Die höhere Förderquote bei transnationalen Projekten bei PRO INNO II hat bereits innerhalb der ersten zwölf Monate zu einem deutlichen Anstieg internationaler Projekte geführt. Zur konkreten Weiterentwicklung hinsichtlich Internationalisierungsunterstützung sollten die Erfahrungen der Ausschreibungen im Rahmen von ERA-SME und CIR-CE berücksichtigt werden, die aktuell durchgeführt werden. Es ist jedoch bereits absehbar, dass eine Prioritätensetzung auf Internationalisierung, wie sie in diesen genannten Aktivitäten vorliegt, für protec 2002+ nicht sinnvoll erscheint. Für den Großteil der österreichischen KMU sind regionale und nationale Wissensträger zunächst einmal die wichtigsten Ansprechpartner. Wenngleich eine deutlichere Förderung einer internationalen Ausrichtung der Zielgruppe ausdrücklich zu unterstützen ist, würde eine vollständige Ausrichtung auf internationalen Technologietransfer jedoch an den Bedürfnissen der Zielgruppe vorbeilaufen.

\section{- Weiterentwicklung der Promotion des Programms}

Trotz der vergleichsweise positiven Resonanz auf die breite Öffentlichkeitsarbeit im Rahmen von protec $2002+$ in den bisherigen drei Ausschreibungsrunden, weist das Programm laut ExpertInnenmeinung eine relativ geringe Bekanntheit insbesondere innerhalb der Zielgruppe der KMU auf. Da es sich bei protec 2002+ auf Grund der ausgeprägten Netzwerk- und Kooperationskomponente um kein Breitenprogramm handelt, kommt einer zielgerichteten und fokussierten Vermarktung des Programms eine bedeutende Rolle zu. Hier gilt es vor allem das Potenzial von Intermediären mit direktem Zugang zu KMU auch vor Ort in den Bundesländern als Multiplikatoren für protec 2002+ stärker zu nutzen. Nicht zuletzt könnte eine intensivere Einbindung regionaler Multiplikatoren und Fördereinrichtungen in die Informations- und Beratungsaktivitäten für protec 2002+ auch dazu beitragen, das bestehende Mobilisierungspotenzial insbesondere in den Bundesländern besser auszuschöpfen. Dabei wäre zu überlegen, in wie weit die entsprechenden Akteure nicht nur als erste Anlaufstelle für potenzielle Antragsteller fungieren, sondern darüber hinaus auch eine so genannte Erstberatung anbieten sowie möglicherweise auch stärkere Unterstützungsleistungen bei der Diffusion und Markteinführung von Projektergebnissen erbringen könnten.

In diesem Zusammenhang wäre etwa auch an eine verstärkte Informationsverbreitung über entsprechende Frauennetzwerke (z.B. FEMtech, IT-Salon Pour Elle etc.) bzw. eine gezielte Ansprache von Frauen in FuE-Abteilungen von Unternehmen zu denken, um zugleich dem Aspekt des Gender Mainstreamings gerecht zu werden und damit die Beteiligung von Frauen an protec $2002+$ zu erhöhen. Nicht zuletzt sei in Hinblick auf die Verbesserung der Kommunikation des Programms auch auf die Notwendigkeit eines einheitlichen Auftritts von protec 2002+ hingewiesen. Zwar finden sich Informationen zu dem Programm auf allen Homepages der an der Programmumsetzung beteiligten Stellen (BMWA, FFG, ERP-Fonds), jedoch gibt es hier keinen Wiedererkennungswert bzw. weist das Programm keine eigene Markenidentität auf. Es wird somit empfohlen, den Außenauftritt von protec 2002+ zu vereinheitlichen und so die Sichtbarkeit des Programms zu stärken.

Zur Sichtbarkeit des Programms tragen auch die in den Programmlinien protec-INNO und protec-NETplus im Rahmen befristeter Calls durchgeführten Ausschreibungsverfahren bei. Für erfolgreiche Fördernehmer bedeuten im Wettbewerb eingeworbene Mittel hohes Prestige für das Projekt und die beteiligten Partner. Eine Erhöhung der Frequenz der Calls, die bisher einmal jährlich stattfinden, in Verbindung mit thematischen Schwerpunktsetzungen, wäre in diesem Zusammenhang anzudenken. 


\section{- Vereinheitlichung des Monitoringsystems}

Das Monitoring und Controlling der Projektumsetzung stellt eine zentrale Informationsquelle für die Begleitung der Programmumsetzung dar, da es den Beitrag der Projekte zur Zielerreichung auf Programmebene im Fokus hat. Das Monitoring der geförderten Projekte erfolgt zurzeit dezentral nach Programmlinie in den unterschiedlichen Monitoringsystemen der beiden Förderabwicklungsstellen. Für das Monitoring auf Programmebene müssen diese Informationen durch die wissenschaftliche Begleitung aus den unterschiedlichen Monitoringsystemen erhoben und zu Programmstatistiken verdichtet werden. Ein einheitliches Monitoringsystem, das für alle Programmlinien gleichermaßen angewendet würde, könnte die Effizienz der Auswertung deutlich erhöhen. Darüber hinaus würde sich dadurch eine leicht modifizierte Aufgabenteilung zwischen Monitoring und Programmmanagement (Förderabwicklung, inkl. -beratung) ergeben: das Leistungsspektrum des Programmmanagements könnte um die Erstellung von Programmstatistiken - als Basis für Monitoringberichte - erweitert werden. In Hinblick auf eine mögliche Weiterentwicklung des Monitoringssystems ließen sich seitens der wissenschaftlichen Begleitung wiederum Überlegungen in Richtung systematisches Monitoring der Ergebnisse von abgeschlossenen Projekten anstellen. Wie die Zwischenevaluierung zeigt, lassen sich aus kompakten "Ex-post Assessments" auch wertvolle Erkenntnisse in Bezug auf "Lessons Learned" bzw. "Good Practice" für die Projektdurchführung und Ausgestaltung der Programmumsetzung ableiten.

\section{Resümee}

Da die vorliegende Evaluation parallel zum (noch) laufenden Programm durchgeführt wurde, bestand eine wichtige Aufgabe darin, Anstöße zur Weiterentwicklung zu geben und damit formativ auf die untersuchte Fördermaßnahme einzuwirken. Hierbei ist als wichtigster Diskussionsanstoß die Reduktion auf zwei Programmlinien zu nennen, wobei die thematischen Schwerpunkte wie oben ausgeführt nicht verringert, sondern erweitert werden sollten. Zudem ist eine engere Abstimmung mit den anderen Bundesprogrammen mit Netzwerklinien anzuregen, um die "Kleinteiligkeit" der Unterstützungsangebote im Bereich Innovationsnetzwerke zu überwinden und damit die Funktionalität der österreichischen Technologietransferförderlandschaft noch effizienter zu gestalten.

Abschließend ist festzuhalten, dass protec 2002+ eine zentrale Funktion darin einnimmt, KMU den Technologietransfer als Option für die Ausweitung ihrer Innovationsaktivitäten näher zu bringen. Das Förderinstrument kann hierbei deutliche Erfolge in der Verringerung von Hemmnissen dieser Zielgruppe vorweisen. Vor dem Hintergrund, dass auch in Zukunft der wissensund innovationsbasierte Wettbewerb weiter deutlich zunehmen wird, und damit die Bedeutung für kleine und mittlere Unternehmen, in einem größeren Ausmaß mit Wissensinstitutionen zu kooperieren, ebenso steigen wird, erscheint eine weitere Förderung von Technologietransfer jedenfalls angebracht. In der vorliegenden Evaluierung konnte aufgrund der kurzen Laufzeit des Programms zwar nur eine begrenzte Zahl bereits abgeschlossener Projekte bewertet werden, bereits diese Zwischenergebnisse weisen jedoch auf einen hohen Zielerreichungsgrad des Programms hin. Betont werden sollte vor allem die ausgezeichnete Governance-Struktur des Programms, die in fast allen Punkten aktuellen Anforderungen der Evaluierungsforschung entspricht und damit als "Best Practice" bezeichnet werden kann. 


\section{Literaturverzeichnis}

Aichinger, K. (2000): Europäische Wettbewerbsfähigkeit und Österreichs Position. WIFO

Beise, M. / Licht, G. / Spielkamp, A. (1995): Technologietransfer an kleine und mittlere Unternehmen. Analysen und Perspektiven für Baden-Württemberg. Baden-Baden: Nomos

BMBWK / BMVIT / BMWA (2005): Österreichischer Forschungs- und Technologiebericht 2005. Lagebericht gem. § 8 (1) FOG über die aus Bundesmitteln geförderte Forschung, Technologie und Innovation in Österreich. Wien: BMBWK, BMVIT, BMWA

BMWA (2005): protec 2002+ Programm zur Förderung des Technologietransfers Richtlinien Endfassung vom Februar 2005. Wien: BMWA

BMWA / ERP-Fonds / TIG (2002): Monitoring- und Evaluierungskonzept, Programmlinien protec-NETplus und protec-INNO. Wien

Technopolis (2003): protec 2002+, Monitoringbericht 1. BMWA, Wien

Technopolis (2004): protec 2002+, Monitoringbericht 2. BMWA, Wien

Technopolis (2005): protec 2002+, Monitoringbericht 3. BMWA, Wien

Dörflinger, A. / Sheikh, S. (2005): Exellence - Eine Frage des Geschlechts. Gender Aspekte in der Forschungs- und Technologieförderung. KMU FORSCHUNG AUSTRIA, Wien

ERP-Fonds (2004): protec-INNO, Programmlinie „Innovationsmanagement, Beratung“ - Leitfaden. Wien

ERP-Fonds (2004): protec-TRANS, Programmlinie „Technologietransfer, Validierung und Demonstration" - Leitfaden. Wien

European Commission (2005): European Trend Chart on Innovation: Annual Innovation Policy Trends and Appraisal Report Austria 2004 - 2005. Brussels: European Commission

Jörg, L. / Jud, T. / Ohler, F. / Pointner, W. / Polt, W. / Zinöcker, K. (2000): Evaluierung des ITFSchwerpunktprogrammes Technologietransfer. Joanneum Research/Technopolis: Wien

Kaufmann, A. / Wagner-Luptacik, P. (2005): Einbeziehung der EU-Strukturprogramme in die nationale FTI-Politik. ARCsys-Bericht, Wien

König, I. / Brustmann, U. (2004): Wie kommt Gender in die Forschung? Leitfaden, Leitweg, Checklist. Wien: BMBWK

Koschatzky, K. (2003): Innovationsnetzwerke als Grundlage regionaler Entwicklungsprozesse. In: Koschatzky, K. (Hrsg.): Die Region - Aktuelle Tendenzen und Entwicklungsstrategien. Stuttgart: IRB

Koschatzky, K. / Kulicke, M. / Zenker, A. (Hrsg.) (2001): Innovation Networks. Concepts and Challenges in the European Perspective. Heidelberg, New York: Physica

Kulicke, M. (2005): Untersuchung der Wirksamkeit von PRO INNO - PROgramm INNOvationskompetenz mittelständischer Unternehmen. Modul 1: Einschätzung der Ergebnisse des Programms PRO INNO. Karlsruhe: Fraunhofer ISI

Lo, V. (2003): Wissensbasierte Netzwerke im Finanzsektor. Wiesbaden: Gabler/DUV. 
Lo, V. / Kulicke, M. / Kirner, E. (2006): Untersuchung der Wirksamkeit von PRO INNO - PROgramm INNOvationskompetenz mittelständischer Unternehmen. Modul 2: Analyse von in den Jahren 2001/2002 abgeschlossenen FuE-Kooperationsprojekten. Stuttgart: IRB.

OECD (2001a): Innovative Networks: Co-operations in National Innovation Systems. Paris.

OECD (2001b): Innovative Clusters. Paris.

Österreichische Forschungsförderungsgesellschaft - FFG (2004): protec-NETplus, Programmlinie „Kooperationen und Netzwerke“ - Leitfaden. Wien

Pleschak, F. (Hrsg.) (2003): Technologietransfer - Anforderungen und Entwicklungstendenzen. Stuttgart: IRB

Rammer, C. / Polt, W. / Egeln, J. / Licht, G. / Schibany, A. (2004): Internationale Trends der Forschungs- und Innovationspolitik - Fällt Deutschland zurück? ZEW Wirtschaftsanalysen Band 73, Nomos

Rat für Forschung und Technologieentwicklung (2005): Strategie 2010. Perspektiven für Forschung, Technologie und Innovation in Österreich. Wien

Reinhard, M. (2001): Wissens- und Technologietransfer in Deutschland - ein langer Weg zu mehr Effizienz. ifo Schnelldienst 4/2001, Jg. 54, 14-17

Reinhard, M. / Schmalholz, H. (1996): Technologietransfer in Deutschland. Stand und Reformbedarf. Berlin, München : Duncker \& Humblot

Schibany, A. / Jörg, L. (2005): Instrumente der Technologieförderung und ihr Mix. Joanneum Research: Wien

Schmoch, U. / Licht, G. / Reinhard, M. (Hrsg.) (2000): Wissens- und Technologietransfer in Deutschland. Stuttgart: IRB

Schrattenecker, I. / Bodisch, U. / Greisberger, H. (2005): Handbuch - Gender in den Forschungs- und Technologieprogrammen des BMVIT. Wien, ÖGUT

Schrattenecker, I. et al. (2004): FEMtech-Studie. Der Frauenanteil in BMVIT-Programmen und Maßnahmen zur Erhöhung der Chancengleichheit in künftigen Ausschreibungen. Wien: ÖGUT

Sheikh, S. / Mandl, I / Radauer, A. / Edler, J. / Lo, V. / Hafner, S. (2004): Evaluierung der Betreuungsstrukturen für das 6 . EU-Rahmenprogramm für Forschung, technologische Entwicklung und Demonstration. Evaluierungsbericht. KMU FORSCHUNG AUSTRIA, Wien / Fraunhofer Institut für Systemtechnik und Innovationsforschung, Karlsruhe

TIG (2003): Vorschriften für das Berichtswesen protec-NETplus. Wien 


\section{Liste der Interviewpartner/innen}

\begin{tabular}{|c|c|c|c|}
\hline & Name & Institution & Funktion \\
\hline \multicolumn{4}{|c|}{ Programmverantwortliche } \\
\hline 1 & Mag. Josef Mandl & $\begin{array}{l}\text { Bundesministerium für Wirtschaft } \\
\text { und Arbeit (BMWA) }\end{array}$ & Programmträger \\
\hline 2 & Silvia Schmöller & $\begin{array}{l}\text { Bundesministerium für Wirtschaft } \\
\text { und Arbeit (BMWA) }\end{array}$ & Programmträger \\
\hline 3 & DI Martin Reishofer & $\begin{array}{l}\text { Österreichische } \\
\text { Forschungsförderungs- } \\
\text { gesellschaft (FFG) }\end{array}$ & Programmabwicklung \\
\hline 4 & Wolfram Anderle & ERP-Fonds & Programmabwicklung \\
\hline 5 & Mag. Leonard Jörg & Technopolis & Wissenschaftliche Begleitung \\
\hline \multicolumn{4}{|c|}{ „Programmunabhängige“ ExpertInnen } \\
\hline 6 & Dr. Kurt Burtscher & $\begin{array}{l}\text { Österreichische } \\
\text { Forschungsförderungs- } \\
\text { gesellschaft (FFG) }\end{array}$ & Externer Experte \\
\hline 7 & DI Arno Klamminger & AT\&S & Juror \\
\hline 8 & MMag. Rudolf Lichtmannegger & $\begin{array}{l}\text { Wirtschaftskammer Österreich } \\
\text { (WKO) }\end{array}$ & Mitglied der Monitoringgruppe \\
\hline 9 & DI Wolfgang Rupp & $\begin{array}{l}\text { Verband der Technologiezentren } \\
\text { Österreichs (VTÖ) }\end{array}$ & Mitglied der Monitoringgruppe \\
\hline 10 & Dr. Michael Stampfer & $\begin{array}{l}\text { Wiener Wissenschafts-, } \\
\text { Forschungs- und } \\
\text { Technologiefonds (WWTF) }\end{array}$ & Mitglied der Monitoringgruppe \\
\hline 11 & Mag. Georg Panholzer & $\begin{array}{l}\text { Bundesministerium für Wirtschaft } \\
\text { und Arbeit (BMWA) }\end{array}$ & Externer Experte \\
\hline 12 & DI Herwig Spindler & $\begin{array}{l}\text { Österreichische } \\
\text { Forschungsförderungs- } \\
\text { gesellschaft (FFG) }\end{array}$ & Mitglied der Monitoringgruppe \\
\hline 13 & DI Dr. Alfred Urban & Innovationsservice Salzburg & Mitglied der Monitoringgruppe \\
\hline 14 & DI Dr. Johann Jäger & $\begin{array}{l}\text { Austrian Cooperative Research } \\
\text { (ACR) }\end{array}$ & Externer Experte \\
\hline 15 & DI Inge Schrattenecker & $\begin{array}{l}\text { Österreichische Gesellschaft für } \\
\text { Umwelt und Technik (ÖGUT) }\end{array}$ & Externe Expertin \\
\hline
\end{tabular}

